Nevada

DOE/NV--654

Environmental

Restoration

Project

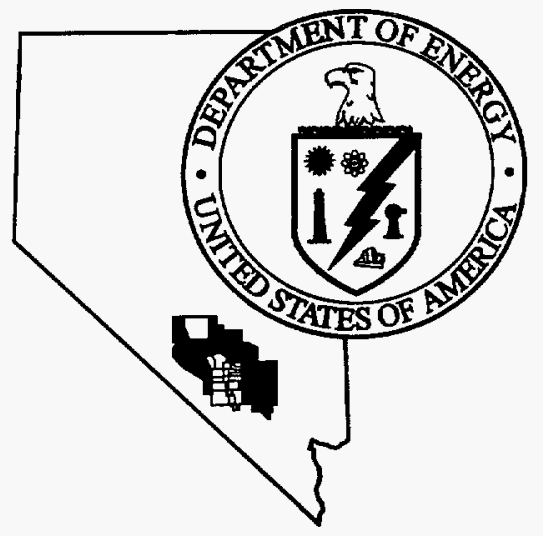

\title{
Closure Report for \\ Corrective Action Unit 486: \\ Double Tracks RADSAFE Area \\ Nellis Air Force Range, Nevada
}

Controlled Copy No.

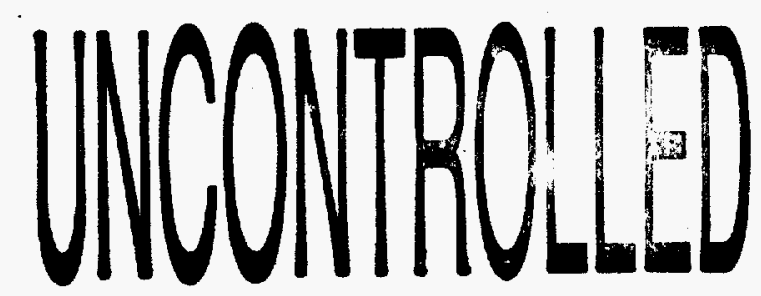

Revision: 0

December 2000

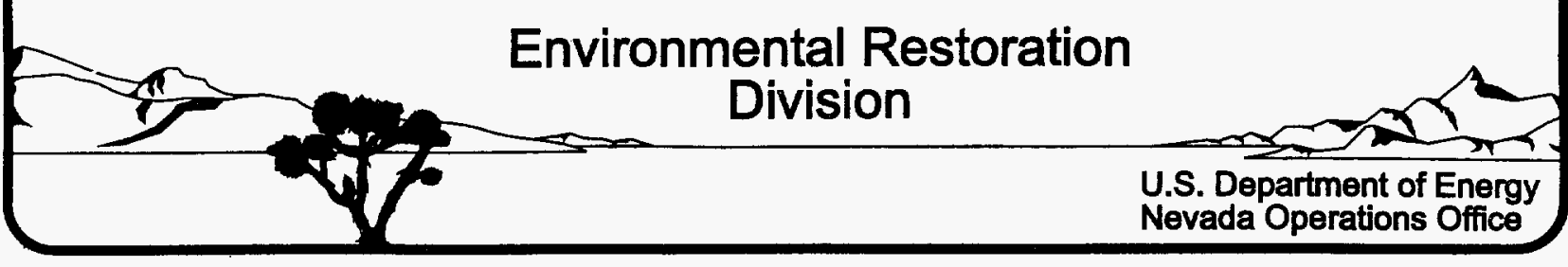




\section{DISCLAIMER STATEMENT}

Reference herein to any specific commercial product, process, or service by trade name, trademark, manufacturer, or otherwise, does not necessarily constitute or imply its endorsement, recommendation, or favoring by the U.S. Government or any agency thereof or its contractors or subcontractors.

\section{AVAILABILITY STATEMENT}

Available for sale to the public from:

U.S. Department of Commerce

National Technical Information Service

5285 Port Royal Road

Springfield, VA 22161-0002

Telephone: $800-553-6847$ or $703-605-6000$

Fax:703-605-6900

E-mail: orders@ntis.fedworld.gov

Online ordering: http://www.ntis.gov/ordering.htm

Available electronically at http://www.doe.gov/bridge

Available for a processing fee to U.S. Department of Energy and its contractors, in paper, from:

U.S. Department of Energy

Office of Scientific and Technical Information

P.O. Box 62

Oak Ridge, TN 37831-0062

Telephone: $865-576-8401$

Fax: 865-576-5728

E-mail: reports@adonis.osti.gov 


\section{CLOSURE REPORT FOR CORRECTIVE ACTION UNIT 486: DOUBLE TRACKS RADSAFE AREA, NELLIS AIR FORCE RANGE, NEVADA}

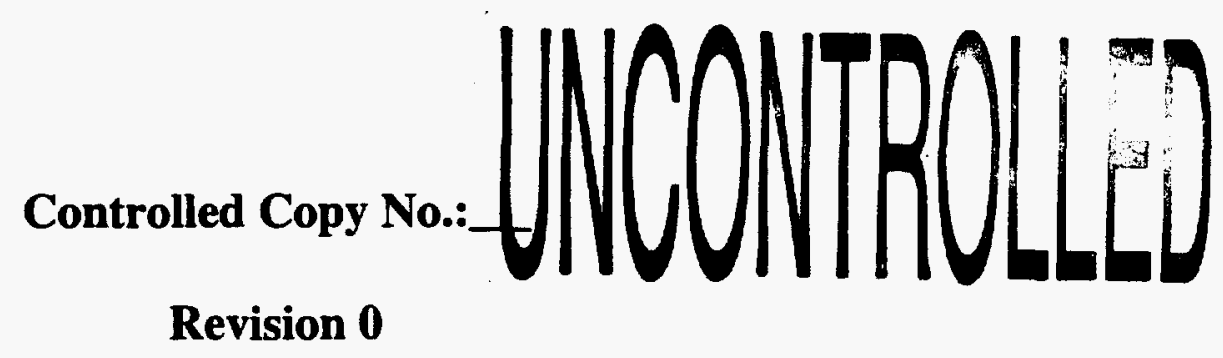

December 2000

Prepared for the U.S. Department of Energy

Nevada Operations Office

under Contract No. DE-AC08-96NV11718 
-

-

.

THIS PAGE INTENTIONALLY LEFT BLANK

7

$-$ 
DOE/NV-654

\section{CLOSURE REPORT FOR CORRECTIVE ACTION UNIT 486: DOUBLE TRACKS RADSAFE AREA, NELLIS AIR FORCE RANGE, NEVADA}

Approved by: $\frac{\text { Shbine T. Curtis }}{\text { for Janet L. Appenzeller-Wing, Project Manager }}$

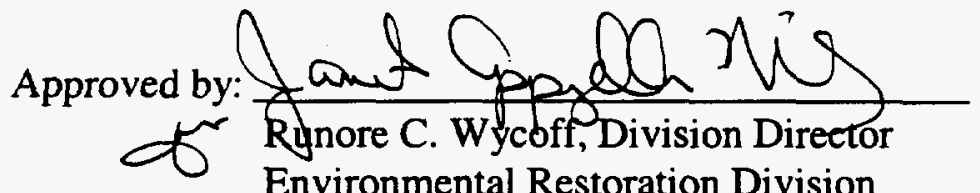

Date: $12 / 26 / 00$

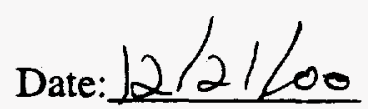





\section{TABLE OF CONTENTS}

ACRONYMS AND ABBREVIATIONS $\ldots \ldots \ldots \ldots \ldots \ldots \ldots \ldots \ldots \ldots \ldots \ldots$ vii

EXECUTIVE SUMMARY $\ldots \ldots \ldots \ldots \ldots \ldots \ldots \ldots \ldots \ldots \ldots \ldots \ldots \ldots \ldots \ldots$

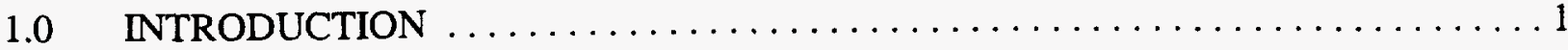

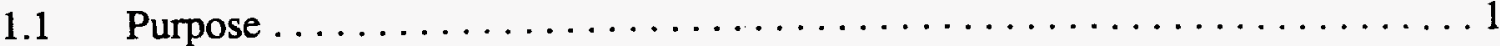

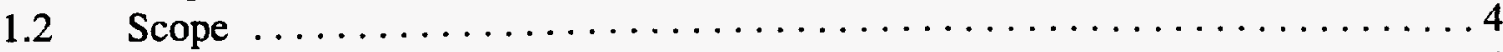

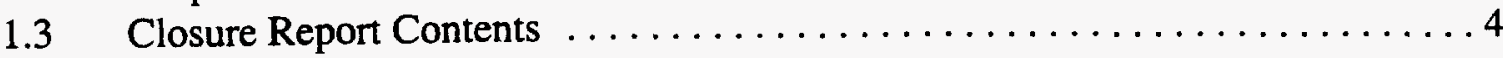

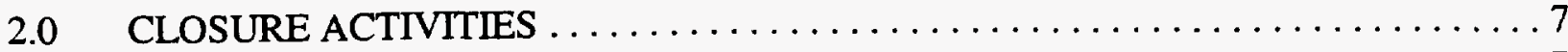

2.1 Description of Corrective Action Activities $\ldots \ldots \ldots \ldots \ldots \ldots \ldots \ldots \ldots$

2.1.1 Preplanning and Site Preparation $\ldots \ldots \ldots \ldots \ldots \ldots \ldots \ldots \ldots$

2.1.2 Excavation of the Area $6 /$ Burial Pit $\ldots \ldots \ldots \ldots \ldots \ldots \ldots \ldots \ldots$

2.1.3 Backfilling and Regrading of Excavations $\ldots \ldots \ldots \ldots \ldots \ldots$

2.1.4 Decontamination of Equipment $\ldots \ldots \ldots \ldots \ldots \ldots \ldots \ldots \ldots$

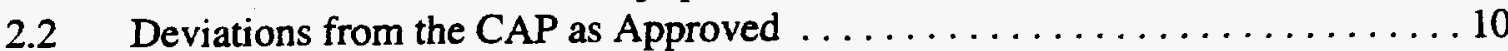

2.3 Corrective Action Schedule as Completed $\ldots \ldots \ldots \ldots \ldots \ldots \ldots \ldots \ldots \ldots$

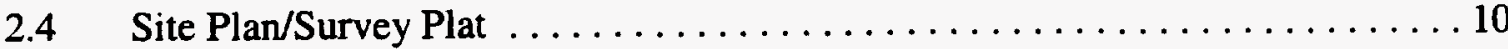

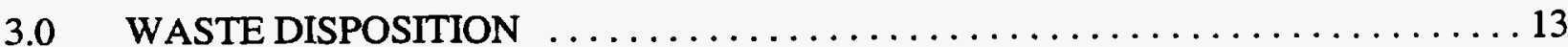

4.0 CLOSURE VERIFICATION RESULTS $\ldots \ldots \ldots \ldots \ldots \ldots \ldots \ldots \ldots \ldots \ldots$

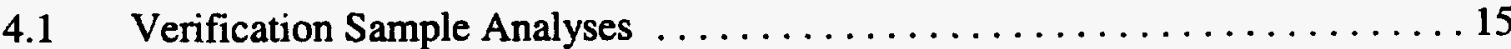

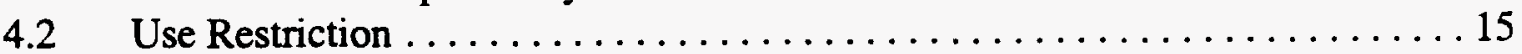

5.0 SUMMARY AND RECOMMENDATIONS $\ldots \ldots \ldots \ldots \ldots \ldots \ldots \ldots \ldots \ldots$

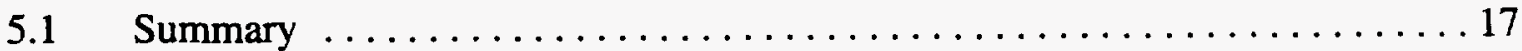

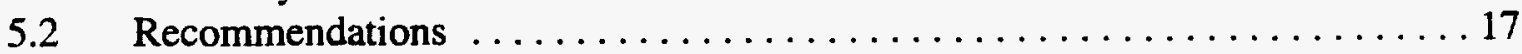

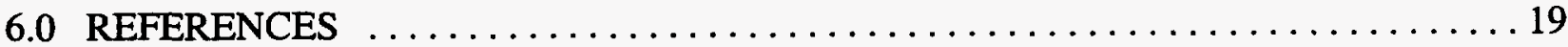




\section{TABLE OF CONTENTS (continued)}

\section{APPENDICES}

Appendix A: Photographs of Closure Activity Work Areas

Appendix B: Waste Disposition Documentation

Appendix C: Verification Sample Analytical Reports

Appendix D: Field Notes

Appendix E: Comment Response Documentation

Distribution List

\section{FIGURES}

Figure 1 - Location Map of CAU 486 Double Tracks RADSAFE Area . . . . . . . . . 2

Figure 2 - Vicinity Map of CAU 486 Double Tracks RADSAFE Area $\ldots \ldots \ldots \ldots \ldots$

Figure 3 - Site Plan of CAU 486 Site Showing Area of Excavation $\ldots \ldots \ldots \ldots \ldots \ldots$

\section{TABLES}

Table 1 - CAU 486 Corrective Action Schedule as Completed 


\section{ACRONYMS AND ABBREVIATIONS}

\begin{tabular}{|c|c|}
\hline bgs & below ground surface \\
\hline $\mathrm{BN}$ & Bechtel Nevada \\
\hline $\mathrm{cm}^{2}$ & Square centimeters \\
\hline CADD & Corrective Action Decision Document \\
\hline CAIP & Corrective Action Investigation Plan \\
\hline CAP & Corrective Action Plan \\
\hline CAS & Corrective Action Site \\
\hline CAU & Corrective Action Unit \\
\hline CR & Closure Report \\
\hline dpm & Disintegrations per minute \\
\hline $\mathrm{DOE} / \mathrm{NV}$ & U.S. Department of Energy, Nevada Operations Office \\
\hline DTRSA & Double Tracks Radiological Safety Area \\
\hline EPA & U.S. Environmental Protection Agency \\
\hline FIDLER & Field Instrument for the Detection of Low Energy Radiation \\
\hline FFACO & Federal Facility Agreement and Consent Order \\
\hline $\mathrm{ft}$ & feet \\
\hline gal & gallon \\
\hline HPGe & High Purity Germanium detector \\
\hline $\mathrm{L}$ & liter \\
\hline $\mathrm{m}$ & meter \\
\hline
\end{tabular}


Date: December 19, 2000

\section{ACRONYMS AND ABBREVIATIONS (continued)}

$\mathrm{m}^{3} \quad$ cubic meter

NDEP

Nevada Division of Environmental Protection

NTS

Nevada Test Site

TTR

Tonopah Test Range

UXO

Unexploded Ordnance

$\mathrm{yd}^{3}$

cubic yard 


\section{EXECUTIVE SUMMARY}

The Double Tracks Radiological Safety Area (DTRSA), Corrective Action Unit (CAU) 486, was clean-closed following the approved Corrective Action Decision Document closure alternative and in accordance with the Federal Facility Agreement and Consent Order (FFACO, 1996). The CAU consists of a single Corrective Action Site, 71-23-001-71DT.

The DTRSA was used during May 1963 to decontaminate vehicles, equipment, personnel and animals from the Double Tracks Test. Double Tracks was one of four storage-transportation tests. The Double Tracks test was conducted in Stonewall Flat, approximately 32 kilometers (20 miles) east of Goldfield, Nevada, on the Nellis Air Force Range. The Double Tracks Test used a single device containing plutonium and depleted uranium and was designed to investigate the characteristics of plutonium-bearing particulate material formed by the non-nuclear detonation of a nuclear weapon.

All facilities associated with the DTRSA operation were removed. Based on available information, the areas of concern at the DTRSA consisted of a decon facility (vehicle decon pad and decon sump) in the southern half of the DTRSA, and a burial pit and former loading/unloading area located in the northern half of the DTRSA.

Based on the results of the Corrective Action Investigation, radiological field screening detected elevated gamma and alpha readings on excavated plastic debris. Swipe surveys taken on the plastic debris detected removable alpha. No contaminants were detected above preliminary action levels in soil samples. The debris excavated during the corrective action investigation was not characterized.

The clean-closure corrective action consisted of excavation, disposal, verification sampling, backfilling, and regrading. Field activities began on May 1, 2000, and ended on May 10, 2000. Soil that was associated with the radiologically contaminated man-made debris was placed into B-25 bins, moved to the designated waste management area where it was scanned, and hauled off-site for disposal. Verification soil samples were collected and analyzed to determine if clean closure had been achieved. Clean borrow soil was hauled to the site and used to backfill the excavation. The excavation was then regraded to promote drainage and minimize ponding of surface water. Since the site is clean-closed, post-closure care is not required. 


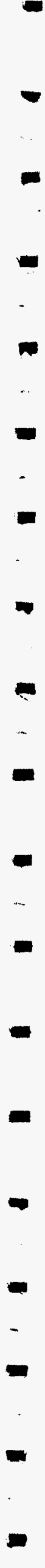




\subsection{INTRODUCTION}

This Closure Report (CR) describes the remediation activities performed at the Tonopah Test Range (TTR) Double Tracks Radiological Safety Area (DTRSA) which was used during May 1963 to decontaminate vehicles, equipment, personnel, and animals from the Double Tracks Test. The DTRSA is identified in the Federal Facility Agreement and Consent Order (FFACO) of 1966 as Corrective Action Unit (CAU) 486 (FFACO, 1996). Remediation of CAU 486 is required under the FFACO (FFACO, 1996). CAU 486 consists of the following Corrective Action Site (CAS) at the TTR (Figure 1):

- CAS 71-23-001: Double Tracks Radiological Safety Area

\subsection{PURPOSE}

The purpose of this CR is to provide documentation of the completed corrective action and to provide data confirming the corrective action. The corrective action was performed in accordance with the approved Corrective Action Plan (CAP) (U.S. Department of Energy [DOE/NV], 1999a) and consisted of clean closure by excavation and disposal.

A complete site history for the CAS is provided in the Corrective Action Investigation Plan (CAIP) U.S. Department of Energy, Nevada Operations Office (DOE/NV, 1998). Corrective action investigation activities were performed from November 16 through December 4, 1998, in two separate phases following the CAIP. Phase I was to locate the previous site features and dimensions using a backhoe. The areas investigated were the former Decontamination Facility, Burial Pit (Area 6) and the loading/unloading location (Area 2) (Figure 2). Phase II was the subsurface investigation consisting of soil sample collection from the three areas of the site. Of the three areas investigated, only the Burial Pit/Area 6, located in the northern half of the DTRSA contained waste. The material consisted of chicken wire, pieces of lumber, cloth, and plastic. A "contaminated material" sticker was observed on the plastic. Radiological field screening conducted on the man-made debris showed an elevated gamma count of 15,600 counts per minute using the Field Instrument for the Detection of Low Energy Radiation (FIDLER). Beta counts were below field screening levels. A swipe sample taken from the plastic detected removable alpha of 283 disintegrations per minute (dpm) per 100 square centimeters $\left(\mathrm{cm}^{2}\right)$. Plutonium and uranium were not detected above minimum detectable activity levels in soil samples collected (DOE/NV, 1999b). The vicinity and area investigated are shown in Figure 2. 
CR-CAU 486

Double Tracks RADSAFE

Section: Introduction

Date: July 21, 2000

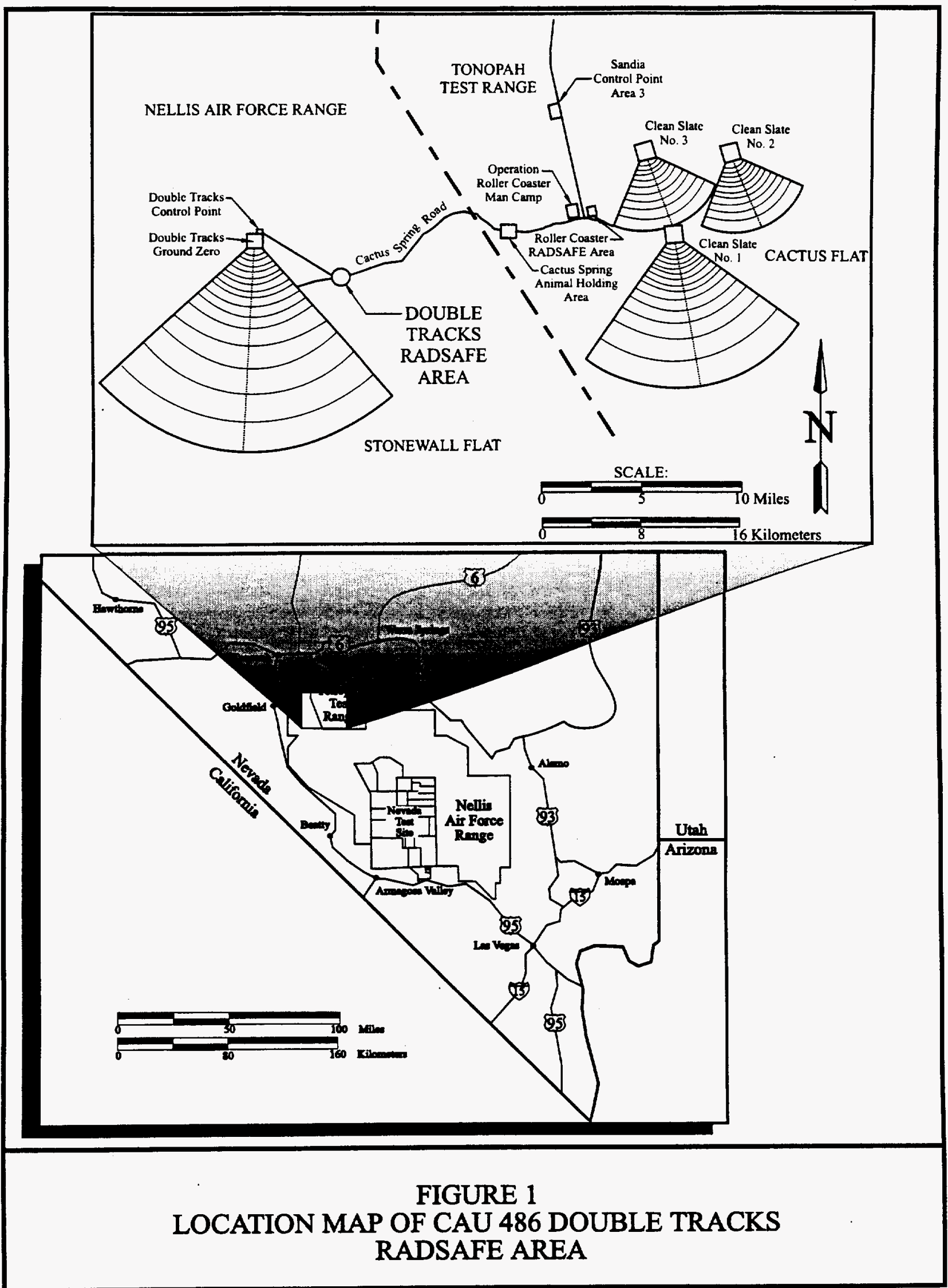




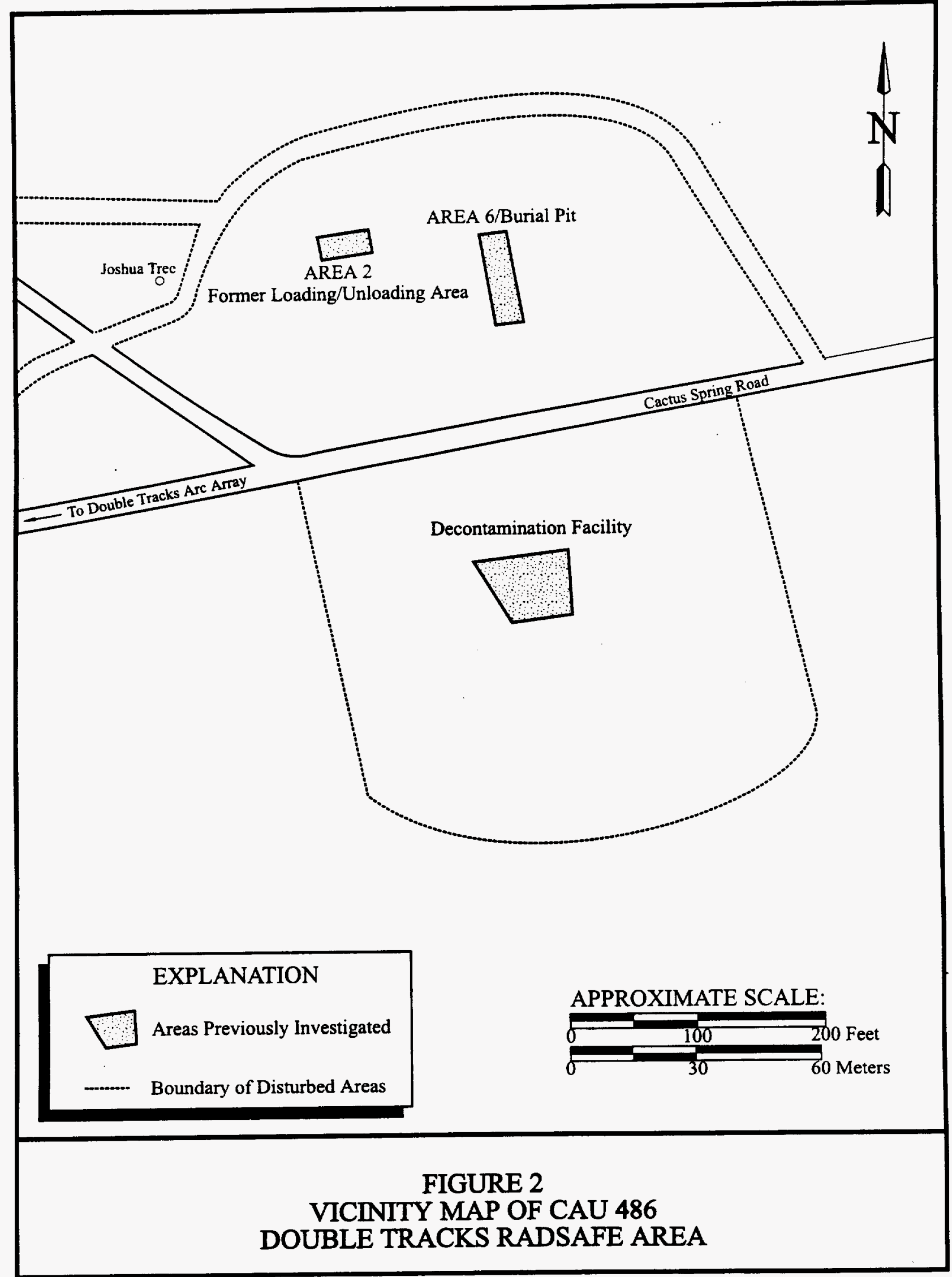




\subsection{SCOPE}

The corrective action as implemented consisted of the following activities:

- Preplanning and site preparation, including preparation of plans and permits, delineation of excavation boundaries, and mobilization of equipment and personnel to the site.

- Excavating impacted material. The excavated material was staged in a designated waste management area at the DTRSA pending transfer to the Nevada Test Site (NTS).

- Inspecting the excavation visually, and collecting verification soil samples for laboratory analysis. Based on results of verification samples, additional excavation was not required.

- $\quad$ Backfilling.

- Regrading the excavation to promote drainage and minimize ponding of surface water.

- Cleaning up the site, including disposal of site surface debris left by previous investigations, and removal of fencing.

\subsection{CLOSURE REPORT CONTENTS}

This document is divided into the following sections in accordance with the approved FFACO CR standardized outline:

- Section 1.0 - Introduction (purpose, scope, contents).

- Section 2.0 - Closure Activities (description, deviations, schedule, site plan).

- Section 3.0 - Waste Disposition (wastes encountered and their appropriate disposal).

- Section 4.0 - Closure Verification Results (laboratory analysis).

- Section 5.0 - Summary and Recommendations.

- $\quad$ Section 6.0 - References.

Certain sections and appendices of this document have been modified from the approved FFACO outline. The following FFACO sections and appendices have not been included or revised as indicated below:

- Use Restriction - Not applicable. The site was clean-closed.

- Closure Certification - Not applicable. 
- As-Built Documentation - Not applicable. No engineered structures were constructed.

- Modifications to the Post-Closure Plan - Not applicable. The site was clean-closed.

The appendices included in this document are provided as follows:

- Appendix A: Photographs of Closure Activity Work Areas.

- Appendix B: Waste Disposition Documentation.

- Appendix C: Verification Sample Analytical Reports.

- Appendix D: Field Notes. 
Closure Report - CAU No. 486

Double Tracks RADSAFE Area

Section: INTRODUCTION

Revision: 0

Date: December 20, 2000

\section{THIS PAGE INTENTIONALLY LEFT BLANK}




\subsection{CLOSURE ACTIVITIES}

This section of the CR details the specific corrective action activities implemented and completed during the closure of CAU 486. This section also provides a detailed schedule of site activities as completed. Photographs showing the work areas before, during, and after closure activities are included in Appendix $\mathrm{A}$.

\subsection{DESCRIPTION OF CORRECTIVE ACTION ACTIVITIES}

\subsubsection{Preplanning and Site Preparation}

Planning documents prepared prior to beginning CAU 486 corrective action activities include the CAP (DOE, 1999a), Field Management Plan (Bechtel Nevada [BN], 2000a), Site Specific Health and Safety Plan (BN, 2000b), a construction work package, and an excavation permit. Aboveground and underground utilities were surveyed prior to starting work. No utilities were found as the site is a remote location of the Nellis Air Force Range. An unexploded ordnance (UXO) survey was conducted by U.S. Air Force Explosive Ordnance Disposal (EOD) specialists prior to starting work. UXO was not found at this location. In addition, a National Environmental Policy Act checklist was prepared and approved. Planned excavation boundaries were identified. A Readiness Review meeting was conducted on April 25, 2000. On April 26, 2000, the pre-job briefing was held and personnel and equipment began the mobilization to the site.

\subsubsection{Excavation of the Area 6/Burial Pit}

The Corrective Action Decision Document (CADD) (DOE/NV, 1999b) identified one location in the Area 6/Burial Pit where a "contaminated material" sticker was observed on a plastic bag. Radiological field screening conducted on the man-made debris showed an elevated gamma count of 15,600 counts per minute using the FIDLER. A swipe sample taken from the plastic detected removable alpha of $283 \mathrm{dpm}$ per $100 \mathrm{~cm}^{2}$. The recommended closure (DOE/NV, 1999b) includes clean closure for Area 6/Burial Pit by excavation and disposal. Since the extent of debris was not verified during the characterization efforts, the CAP

(DOE/NV, 1999a) called for the excavation of three trenches within the burial pit to a minimum depth of 1.5 meters $(\mathrm{m})$ (5.0 feet [ft]) below ground surface (bgs) using backhoe equipment. A central trench, approximately $23 \mathrm{~m}$ (75 ft) long, was to be excavated; and two additional trenches, approximately $9 \mathrm{~m}$ (30 ft) long, were to be excavated on either side of the central trench. Once debris was observed in a trench, the trench was to be extended laterally and vertically to delineate the extent of the debris to be removed.

On May 1, 2000, the mobilization and the Explosive Ordnance Disposal survey were completed. On May 02, 2000 the excavation of the Area 6/Burial Pit was begun. Using a backhoe, the first $0.6 \mathrm{~m}$ ( $2 \mathrm{ft}$ ) of soil was excavated and stockpiled as clean fill at the south end of the location. 
Date: December 20, 2000

At a depth of $0.76 \mathrm{~m}$ ( $2.5 \mathrm{ft})$, scrap metal and plastic debris was uncovered. No elevated radioactive readings were detected. A second stockpile was started, containing soil and manmade debris.

At $0.91 \mathrm{~m}$ (3.0 ft) bgs, wire, wood, and plastic was observed. No elevated radioactive readings were detected. The floor of the excavation at this point averaged $1.06 \mathrm{~m}(3.5 \mathrm{ft})$. A "pothole" was dug at the middle borehole, "NB3", as identified in the CADD (DOE/NV, 1999b). Total depth of the pothole was $2.0 \mathrm{~m}$ to $2.1 \mathrm{~m}(6.5 \mathrm{ft}$ to $7.0 \mathrm{ft} \mathrm{bgs})$. Pothole NB3 was obviously a debris-layer portion of the Burial Pit excavation, however there was only a slight elevation in radiological activity ( $\pm 200 \mathrm{dpm}$ ). The first B-25 bin was moved into the Exclusion Zone (EZ) and the first lift of soil and debris was placed into the bin. The Remote Sensing Laboratory personnel installed the High Purity Germanium (HPGe) detector over the bin and surveyed for radiological activity. No elevated radiological activity readings were detected. The second and third lifts were added to the bin with each lift checked with the HPGe detector. No elevated radiological activity readings were detected with any of the three instruments used on the project (Electra, FIDLER, and the HPGe). All debris at this point was trash, not hotline material as expected. The width of the "6NT1 trench", identified in the CADD (DOE/NV, 1999b) as the trench containing "radiologically contaminated debris" was extended $1.82 \mathrm{~m}(6.0 \mathrm{ft})$. One plastic bag was found with Radiological Control tape ("Contaminated Material") attached. The debris in the plastic bag consisted of tape, paper, food packaging, etc. No hotline waste was found. No elevated radiological activity readings were detected from this debris. The length and width of the excavation were extended and three more bags of trash were recovered with no elevated radiological activity readings.

One B-25 was set off to the side for any obviously radiological material or debris indicating elevated radiological activity. Also, in order to conserve the B-25 containers the decision was made to separate the majority of the clean soil from the other debris. Two B-25 boxes were staged, one being filled with obvious or indicating radiological debris, and the other filled with segmented material such as plastic, wire, wood, etc. As the excavation progressed, besides the abundant trash that was segmented and placed into the "trash" B-25, numerous plastic bags containing hotline trash and some loose debris with elevated radiological activity (300 to 4,000 counts/second [FIDLER]) was placed in the radiological material bin. As the bins were filled they were moved to the Contaminated Storage Area where they were surveyed, weighed, and placarded, pending transport to the NTS.

The completed excavation was visually inspected, all debris was removed, and no staining or discoloration was observed.

Verification soil samples collected in the former Area 6/Burial Pit area are numbered sequentially using the following nomenclature: CAU4860001, CAU4860002, etc. The samples were collected from the bottom of the Area 6/Burial Pit excavation. Details of soil sampling, handling, analyses, and results are discussed in Section 4.1. 
On May 10, 2000 approximately 9 cubic meters $\left(\mathrm{m}^{3}\right)$ (12 cubic yards [yd $\left.\left.\mathrm{d}^{3}\right]\right)$ of waste soil and compactable trash was transported off the TTR for disposal at the NTS. Waste disposition is discussed in Section 3.0.

\subsubsection{Backfilling and Regrading of Excavations}

The backfilling operation began on May 8, 2000. Due to the trash segmentation efforts, the source of backfill material (Area 3 Borrow Pit) identified as a pre-project requirement was no longer required. Stockpiled material at the site was sufficient to complete the backfill operation in order to level the area to the existing grade, restore drainage, and minimize ponding of surface water.

The stockpiled soil was subsequently placed in the excavation, and wheel compacted using the backhoe, forklift, or water truck, as needed. Final site regrading was completed on May 10, 2000.

\subsubsection{Decontamination of Equipment}

After the final load of radiologically contaminated debris was handled, equipment that had contacted the debris (backhoe bucket and shovel) was cleaned by first brushing off visible residue, then washing with a laboratory-grade detergent solution, and followed by a tap water rinse. Equipment decontamination was performed over a B-25 bin in order to contain rinsate in the excavated debris. Less than 4 liters [L] (1 gallon [gal]) of detergent solution and water rinse were used and was completely absorbed (no free liquid) into the waste debris.

Soil sampling scoops were decontaminated before field mobilization using a laboratory-grade detergent solution, an isopropanol rinse and deionized water rinses. 


\subsection{DEVIATIONS FROM THE CAP AS APPROVED}

No significant deviations occurred from the approved scope of work as outlined in the CAP (DOE, 1999a). The following minor deviations occurred from the approved scope of work:

- Since the extent of debris was not verified during the characterization efforts, three trenches were to be excavated within the burial pit to a minimum depth of $1.5 \mathrm{~m}(5.0 \mathrm{ft})$ below ground surface using backhoe equipment. A central trench, approximately $23 \mathrm{~m}$ ( $75 \mathrm{ft}$ ) long, was to be excavated. Two additional trenches,

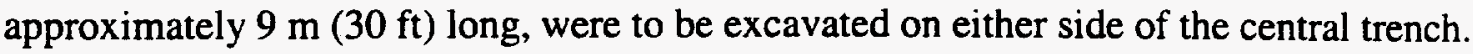
Once debris was observed in a trench, the trench was to be extended laterally and vertically to delineate the extent of the debris to be removed. In actuality, one large excavation resulted from chasing debris, which would most likely correspond to the original Area 6/Burial Pit configuration.

- Verification soil samples were collected in fewer locations than planned. It was estimated that a total of fourteen samples would be collected including one duplicate and one equipment blank. The number of samples collected was contingent on the volume of debris encountered in the three trenches. Since one large excavation resulted from the debris removal operation, seven samples were sufficient to adequately represent the floor of the excavation. A duplicate sample and an equipment blank were also collected. Sampling locations were documented in the field and reported in the Technical Lead's log book.

- The actual volume of generated waste varied from the planned volume. A smaller volume of waste soil and compactable hotline trash was disposed $\left(9 \mathrm{~m}^{3}\left[12 \mathrm{yd}^{3}\right]\right)$ than planned $\left(22.9 \mathrm{~m}^{3}\right.$ [30 $\left.\left.\mathrm{yd}^{3}\right]\right)$ because of the efforts to segment this material from the otherwise clean soil.

\subsection{CORRECTIVE ACTION SCHEDULE AS COMPLETED}

The corrective action field activities began on May 1, 2000, and were completed on May 10, 2000. A corrective action schedule as completed is provided in Table 1.

\subsection{SITE PLAN/SURVEY PLAT}

Survey data were not required for this closure. Because engineered construction was not required as part of this closure, as-built drawings are not included in this Closure Report. Figure 3 shows the location of the verification samples collected and the condition of the site following closure activities. 


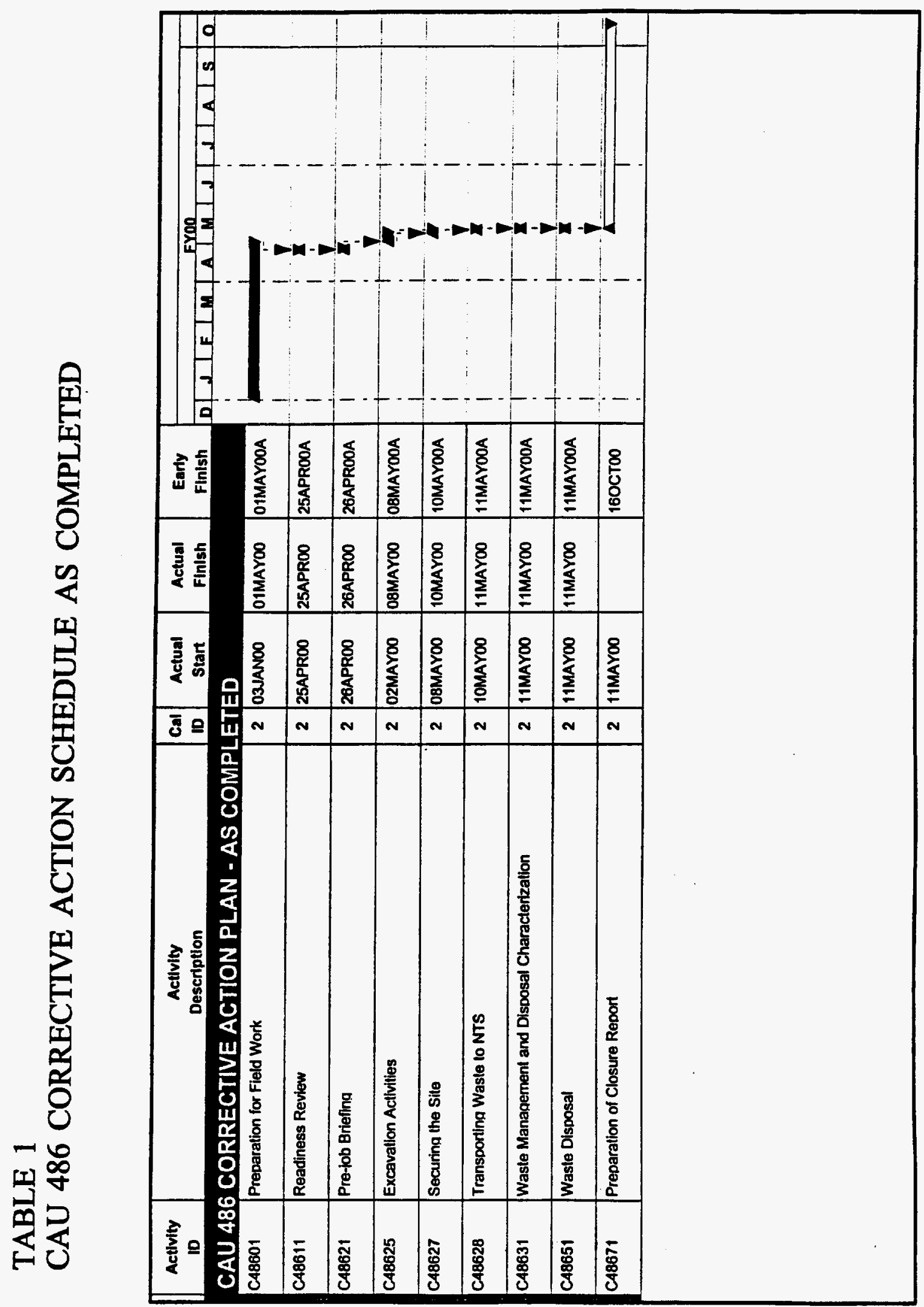




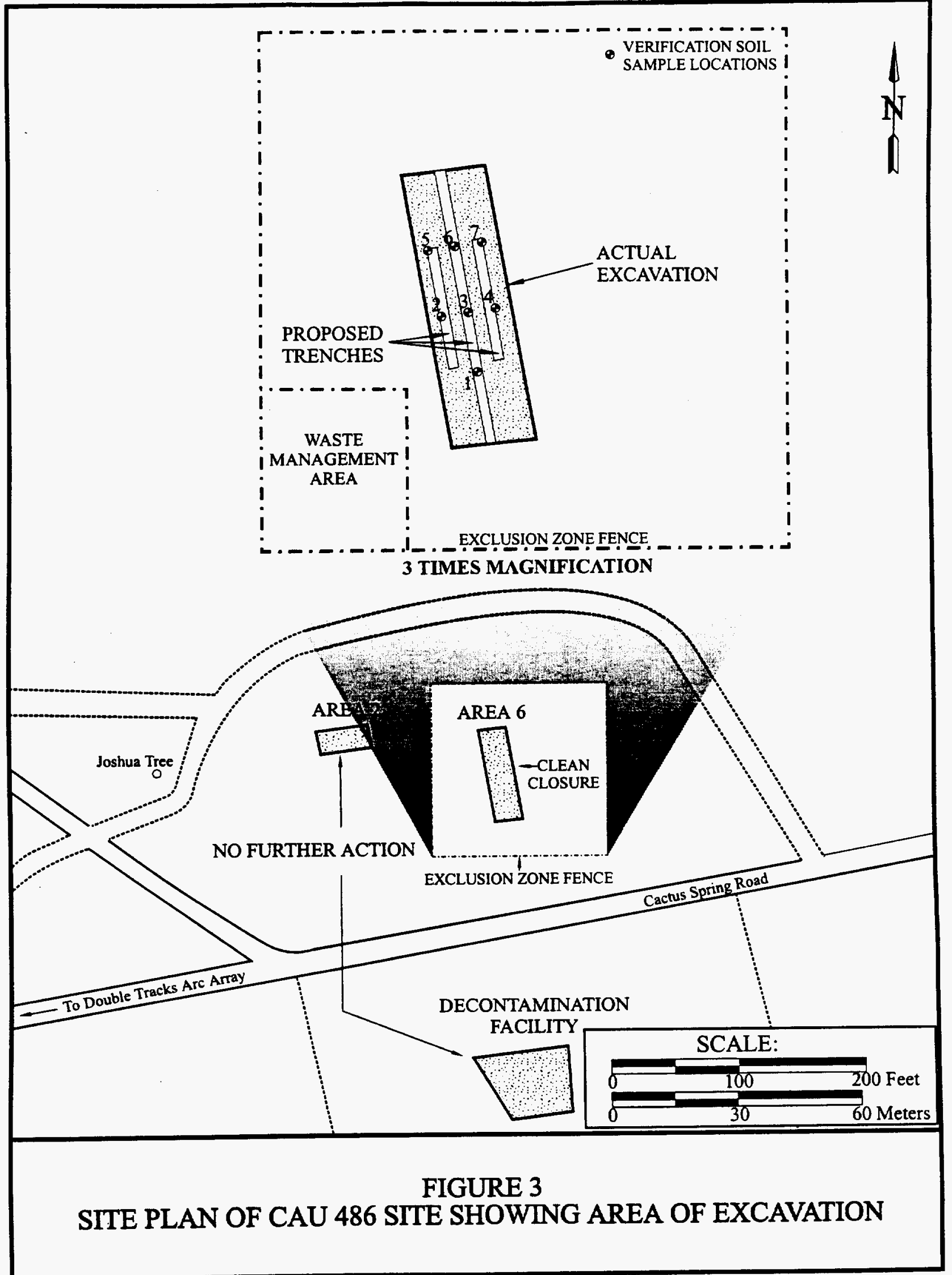


Wastes generated during the closure of CAU 486 were disposed as follows:

- Approximately $9 \mathrm{~m}^{3}\left(12 \mathrm{yd}^{3}\right)$ of waste soil and compactable hotline trash was staged onsite in a designated Waste Management Area and then transported off-site as NonRegulated Waste and received at the NTS Radioactive Waste Management Site in Area 5 for disposal on May 11, 2000.

Waste disposition records, including the Uniform Hazardous Waste Manifest and NTS landfill documents, are available in NTS Waste Operations files and are summarized in Appendix B. 



\subsection{CLOSURE VERIFICATION RESULTS}

\subsection{VERIFICATION SAMPLE ANALYSES}

Verification soil samples were collected from seven locations after the excavation reached designated boundaries, removed all debris, and no visible staining was observed. The samples were collected with decontaminated stainless steel sampling scoops and placed in labeled sample containers and then secured with custody seals. A FIDLER was used to help determine if soil samples exceeded background levels. The containers were placed in an ice-filled chest, transported under chain-of-custody to the Analytical Services Laboratory in Mercury, Nevada, for analyses. Samples from all seven locations were analyzed for 20-minute gamma spectroscopy (U.S. Environmental Protection Agency, 1996).

Since no other constituents of concern were identified during site characterization activities, verification samples were only analyzed for 20 -minute gamma spectroscopy. The analytical results indicate that no man-made radiation was detected above action levels and confirm that no further excavation is needed. The analytical reports are found in Appendix C.

\subsection{USE RESTRICTION}

A clean closure was performed at this CAS. Land use is unrestricted. A Post-Closure Plan is not necessary for this site. 
Closure Report - CAU No. 486

Double Tracks RADSAFE Area

Section: CLOSURE VERIFICATION

Revision: 0

Date: December 19, 2000

\section{THIS PAGE INTENTIONALLY LEFT BLANK}




\subsection{SUMMARY AND RECOMMENDATIONS}

\subsection{SUMMARY}

The following site closure activities were performed at the CAU 486 site located at the TTR and are documented in this report:

- $\quad$ Preplanning and site preparation.

- Excavating and removing waste.

- Characterizing waste on-site for disposal.

- Collecting verification soil samples.

- Backfilling excavation with stockpiled soil and regraded to promote drainage and minimize ponding of surface water.

- Disposing of excavated materials following applicable federal, state, and DOE regulations.

- The field closure activities conducted at CAU 486 were completed following the approved CAP (DOE/NV, 1999a) with only minor deviations as specified in Section 2.2.

\subsection{RECOMMENDATIONS}

Since the clean closure for CAU 486 has been completed following the Nevada Division of Environmental Protection (NDEP) approved CAP (DOE/NV, 1999a) as documented in this CR, the DOE requests:

- A Notice of Completion be provided by the NDEP to DOE for the closure of CAU 486 (CAS 71-23-001-71DT).

- CAU 486 be moved from Appendix III to Appendix IV of the FFACO "Closed Corrective Action Units." 



\subsection{REFERENCES}

BN, see Bechtel Nevada.

Bechtel Nevada, 2000a. Field Management Plan for Corrective Action Unit 486: Double Tracks RADSAFE Area, Nellis Air Force Range, Nevada.

Bechtel Nevada, 2000b. Site Specific Health and Safety Plan for Corrective Action Unit 486: Double Tracks RADSAFE Area, Nellis Air Force Range. Nevada.

DOE/NV, see U.S. Department of Energy.

EPA, see U.S. Environmental Protection Agency

FFACO, see Federal Facility Agreement and Consent Order.

Federal Facility Agreement and Consent Order of 1996. Prepared by Nevada Division of Environmental Protection, U.S. Department of Energy, and U.S. Department of Defense.

U.S. Department of Energy, Nevada Operations Office, 1999a. Corrective Action Plan for Corrective Action Unit 486: Double Tracks RADSAFE Area, Nellis Air Force Range, Nevada, DOE/NV--584, Las Vegas, NV.

U.S Department of Energy, Nevada Operations Office, 1998. Corrective Action Investigation Plan for Corrective Action Unit 486: Double Tracks RADSAFE Area, Nellis Air Force Range. Nevada. DOE/NV--523, Las Vegas, NV.

U.S. Department of Energy, Nevada Operations Office, 1999b. Corrective Action Decision Document for Corrective Action Unit 486: Double Tracks RADSAFE Area, Nellis Air Force Range. Nevada. DOE/NV--555, Las Vegas, NV.

U.S. Environmental Protection Agency, 1996, Test Methods for Evaluating Solid Waste. Physical/Chemical Methods, EPA Publication SW-846, Third Edition. 




\section{APPENDIX A}

\section{PHOTOGRAPHS OF CLOSURE ACTIVITY WORK AREAS}


NOTE: 45 PHOTOGRAPHS EXIST OF THIS CLOSURE, ATTACHED IS A SELECTION 
THIS PAGE INTENTIONALLY LEFT BLANK

$\mathbf{a}$

$-$

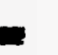<smiles></smiles> 


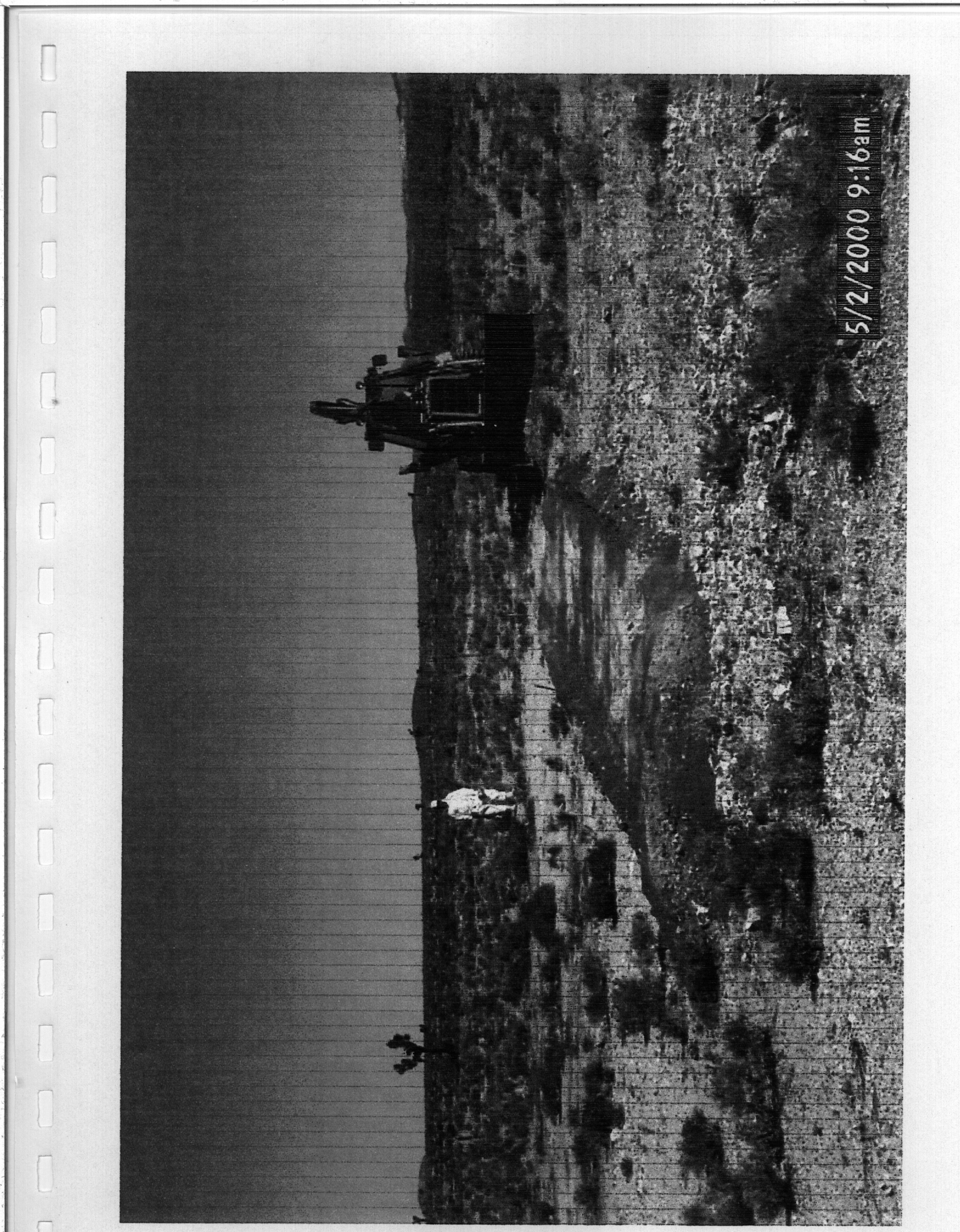




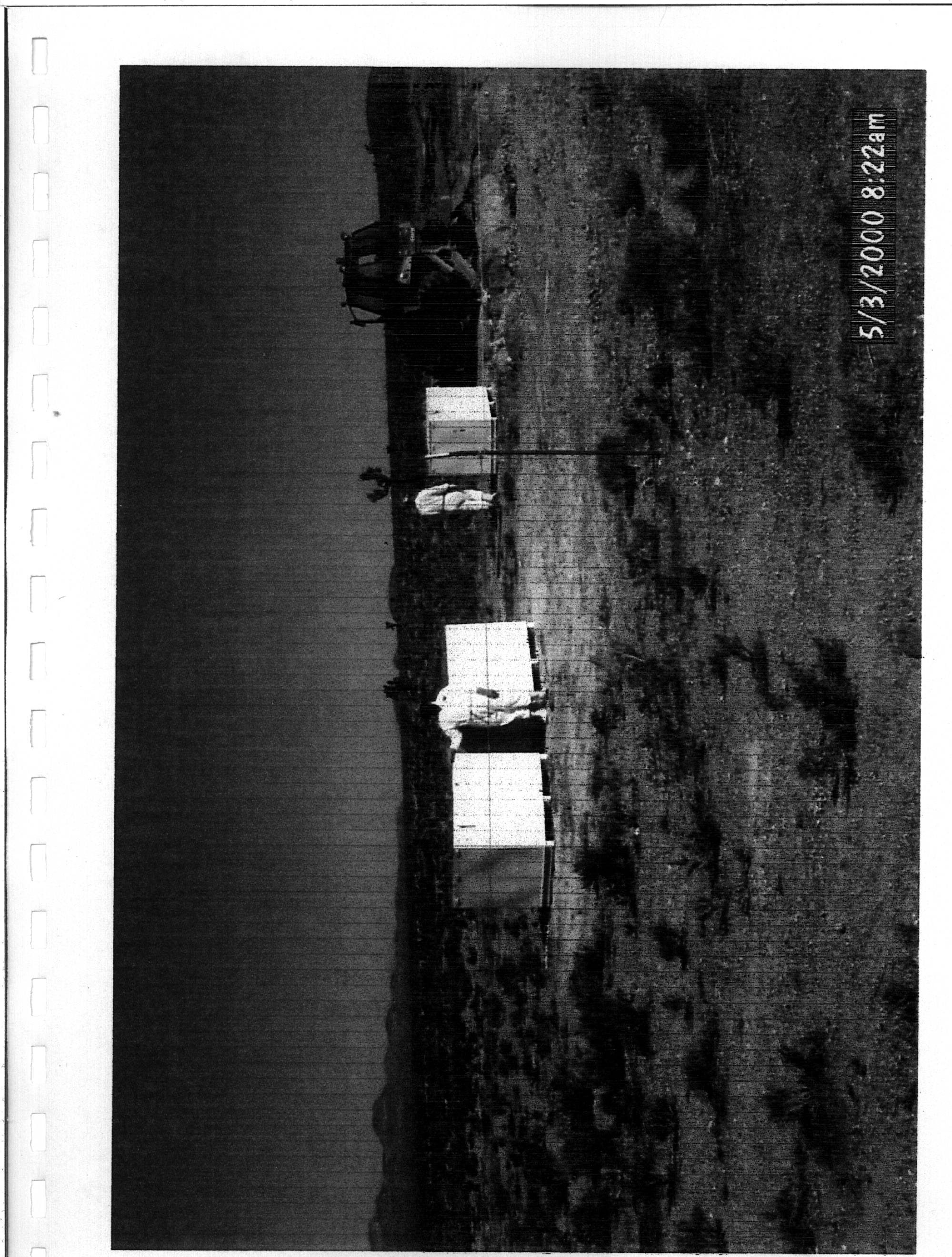




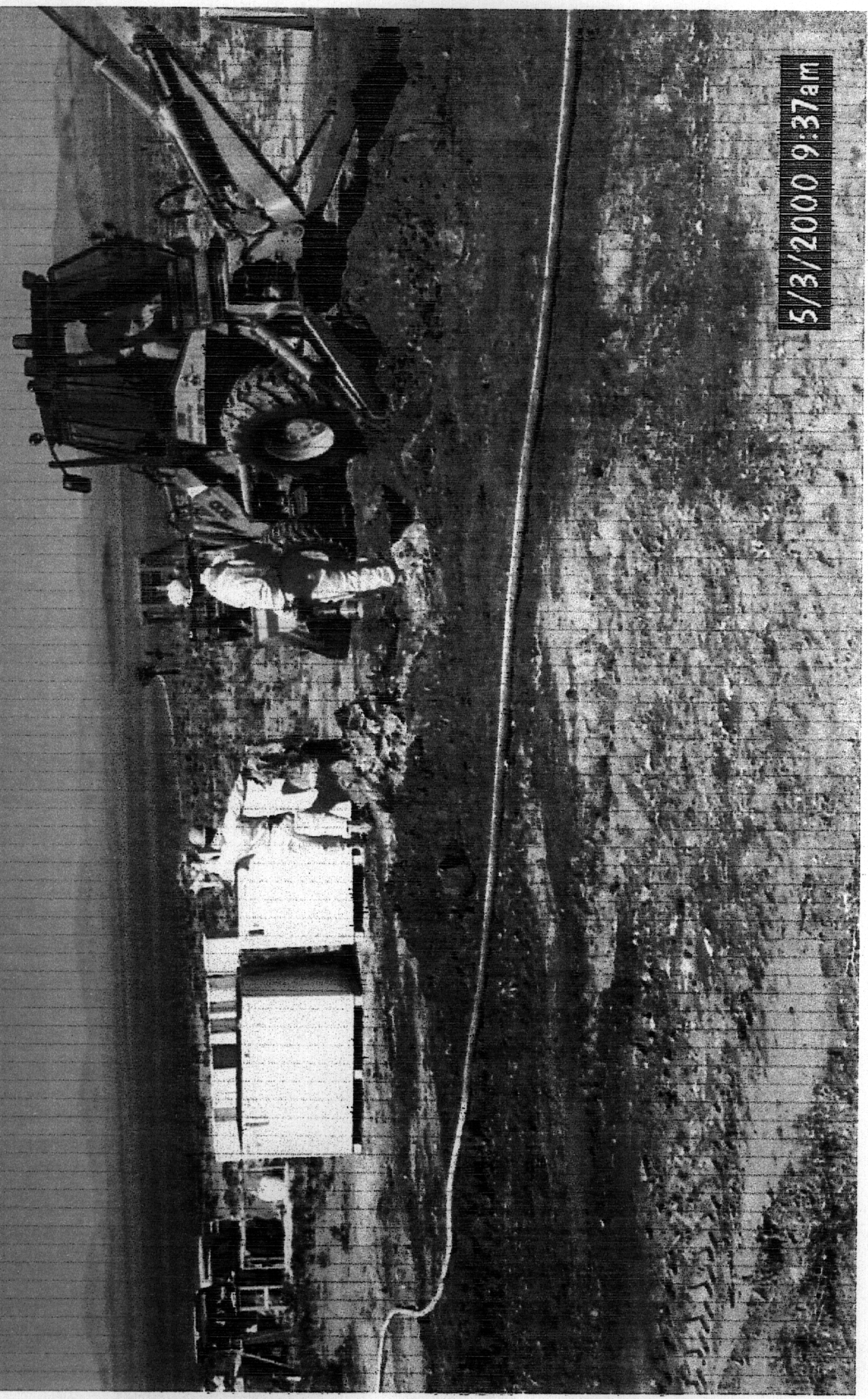




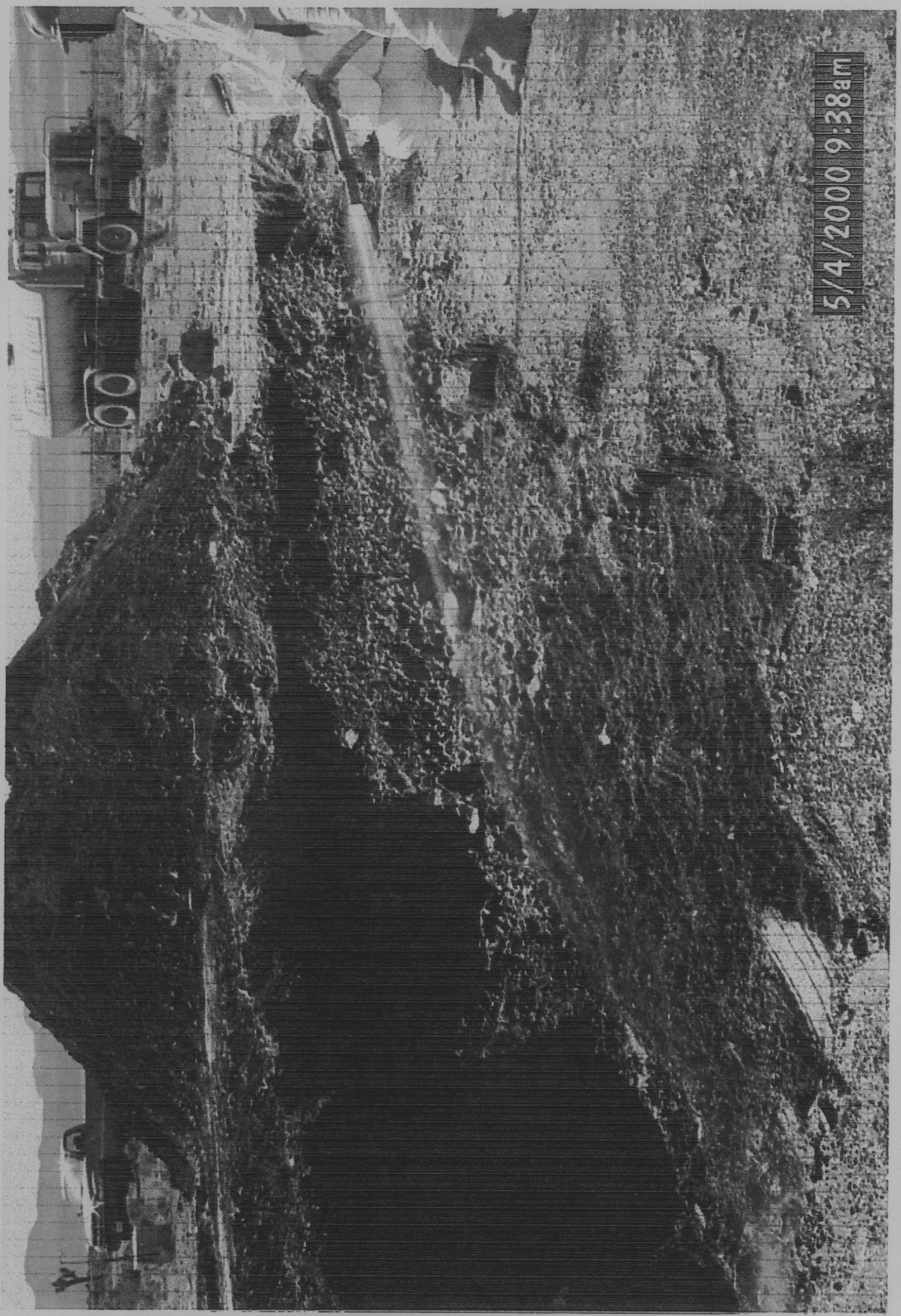




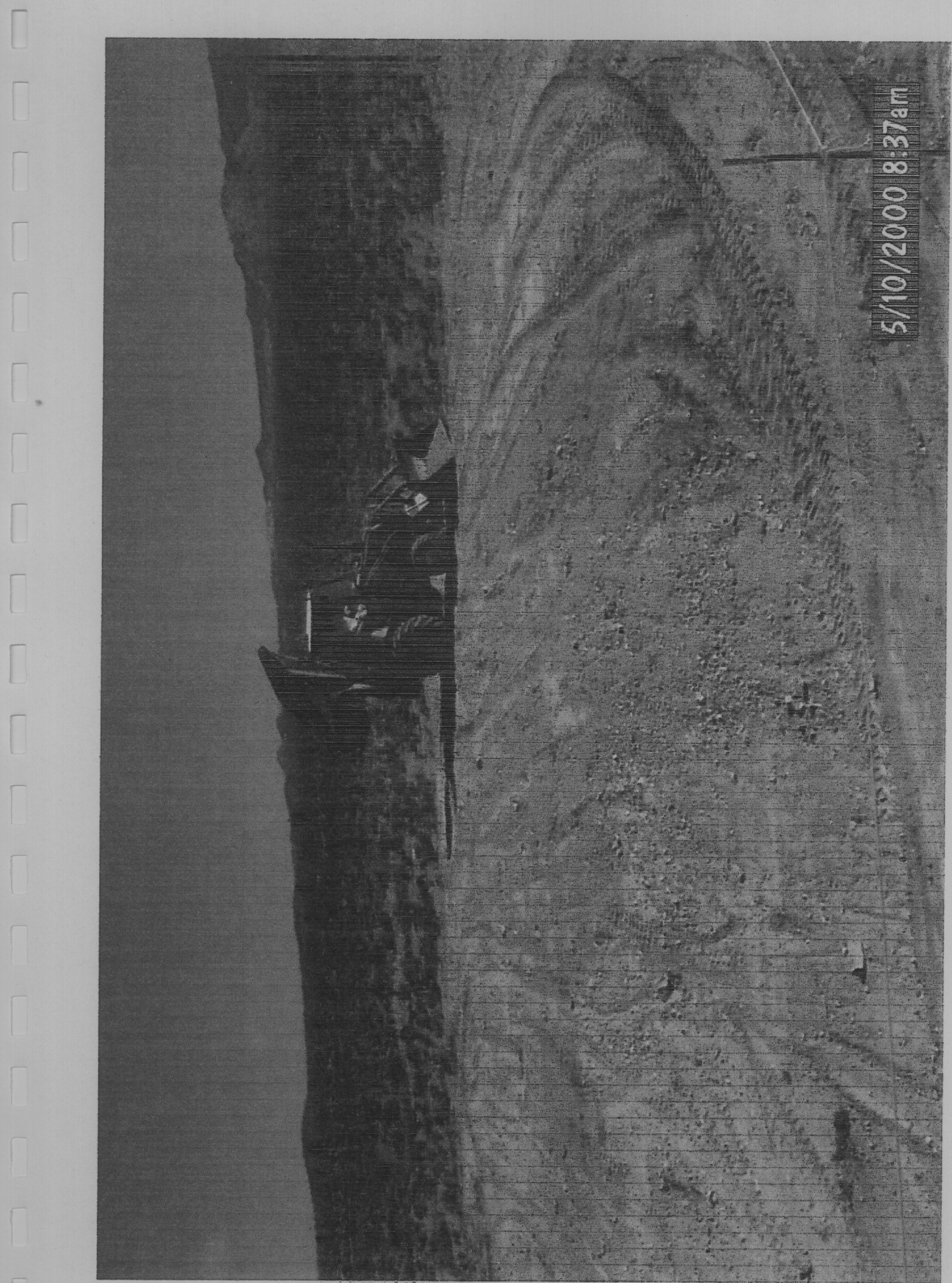




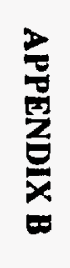




\section{APPENDIX B}

\section{WASTE DISPOSITION DOCUMENTATION}




\section{THIS PAGE INTENTIONALLY LEFT BLANK}

س 


\section{CERCTIFICATE OF DISPOSAL}

This is to certify that the Double Tracks (CAU 486) Waste Stream Number LRY5LLFY00006, MEF Number F00006, with package numbers 982810, 982774, 982817, and 982803 were shipped and received at the Nevada Test Site Radioactive Waste Management Site in Area 5 for disposal as stated below.

Shipped by:

William C. Nicosia

Print Name

Liel C. Hear-

Signature

Received by:

Kaved E. Williams

Print Name

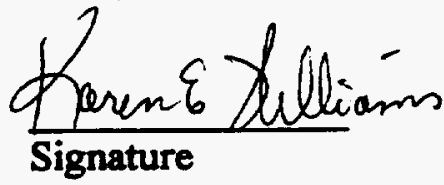

Bechtel Nevada Waste Control

Organization

Scientist

Title

$\frac{5 \cdot 10.00}{\text { Date }}$

Scientisi-

Title

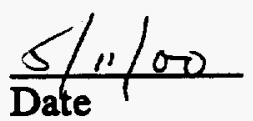




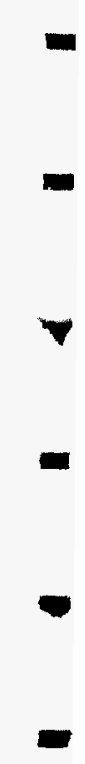




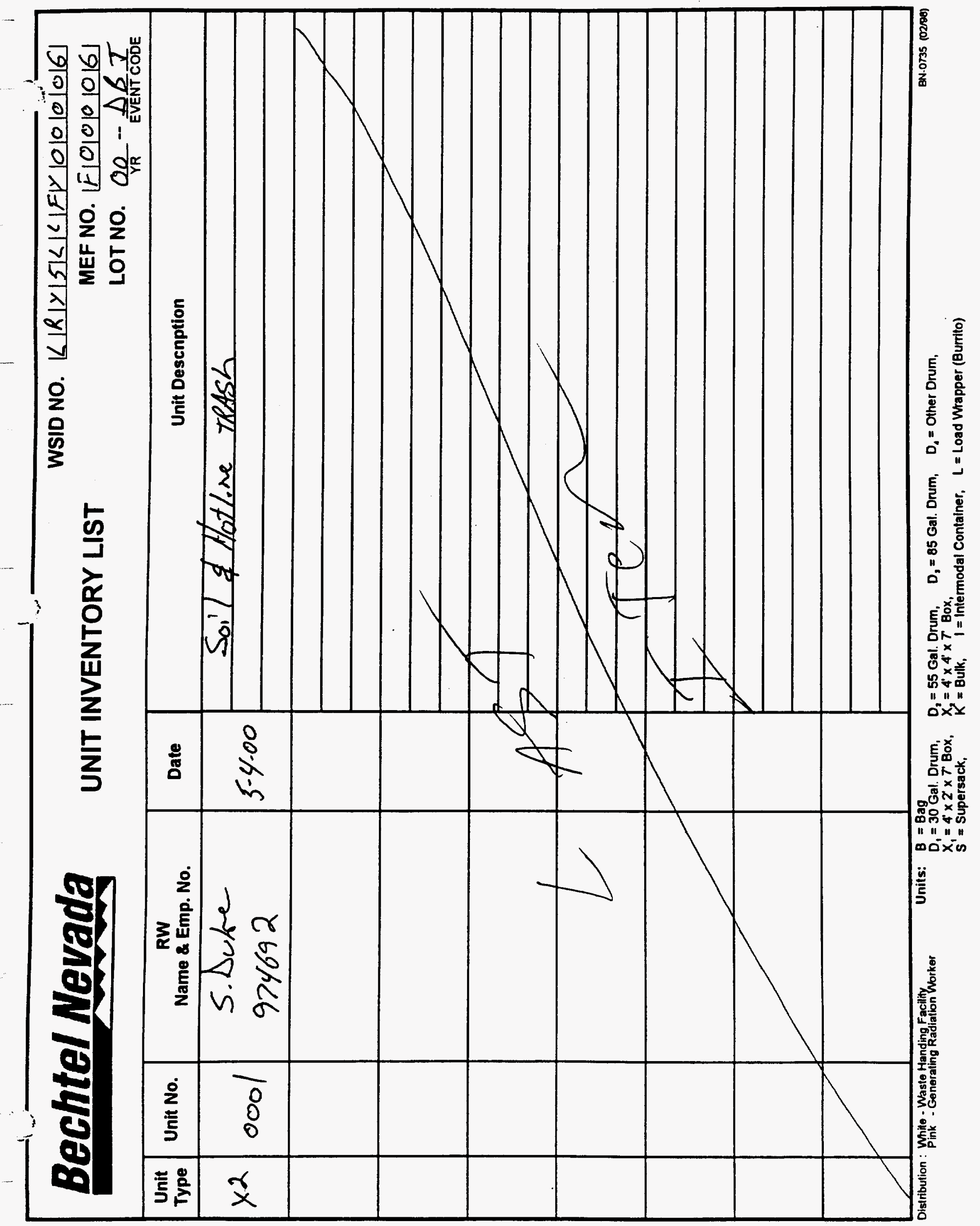




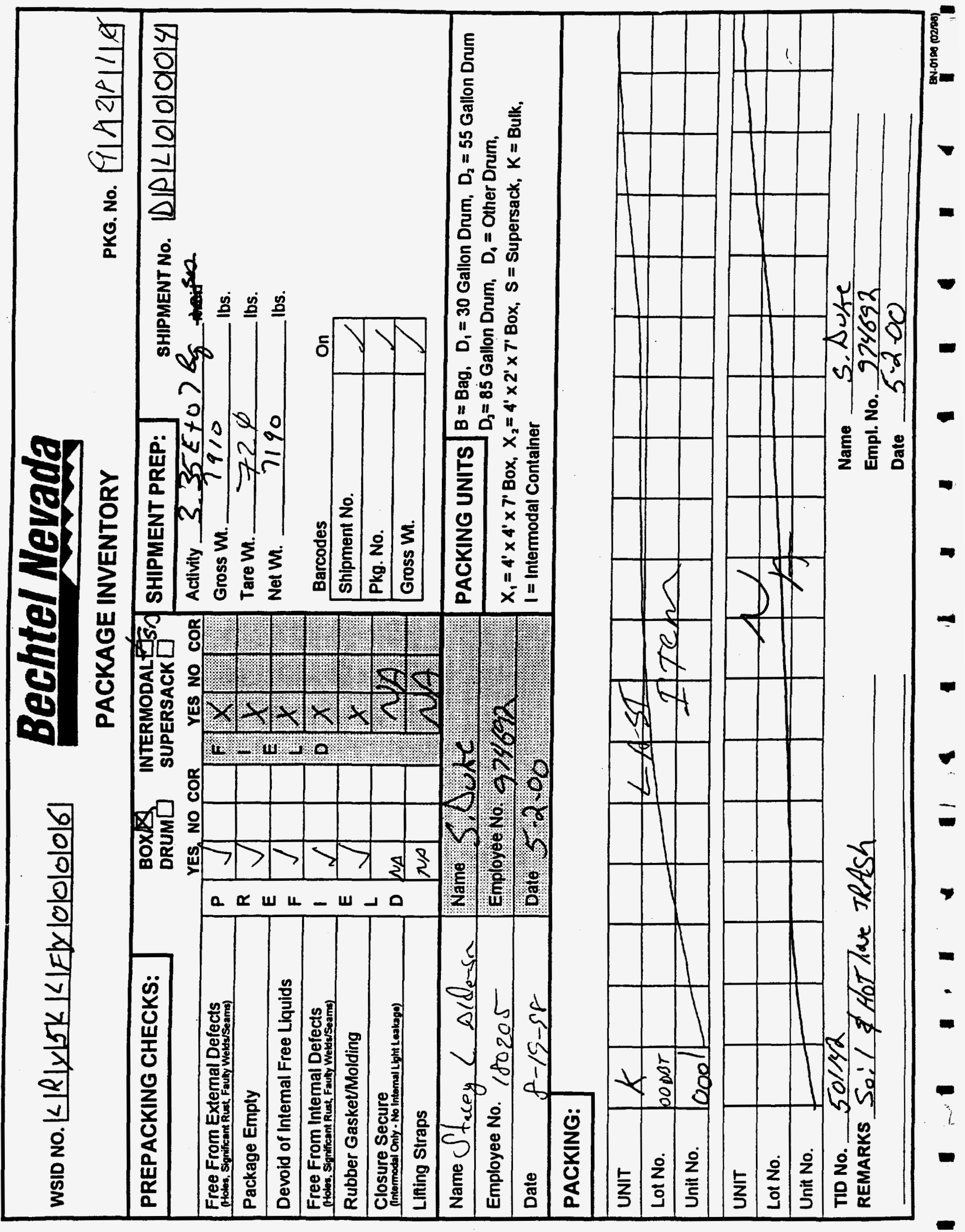




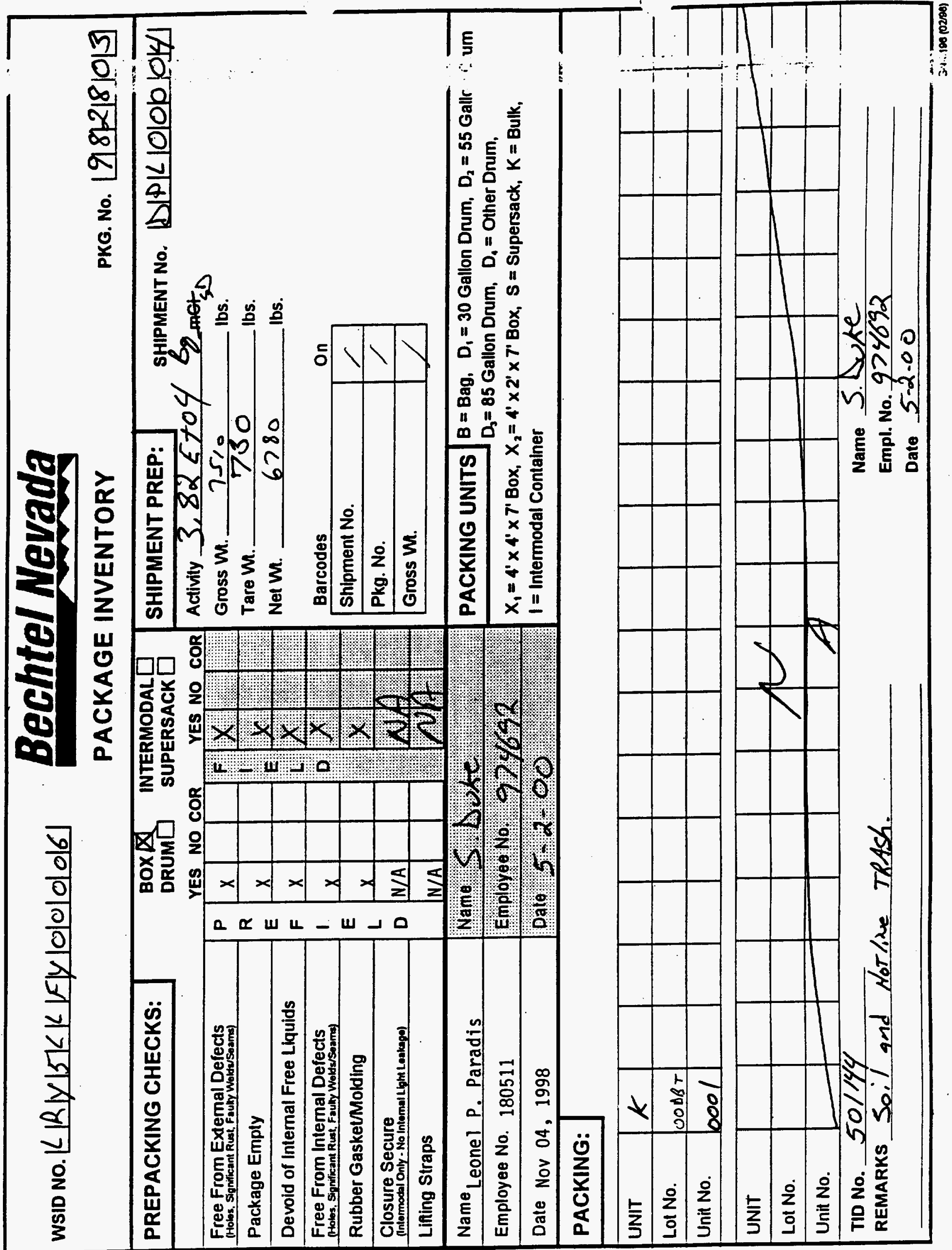




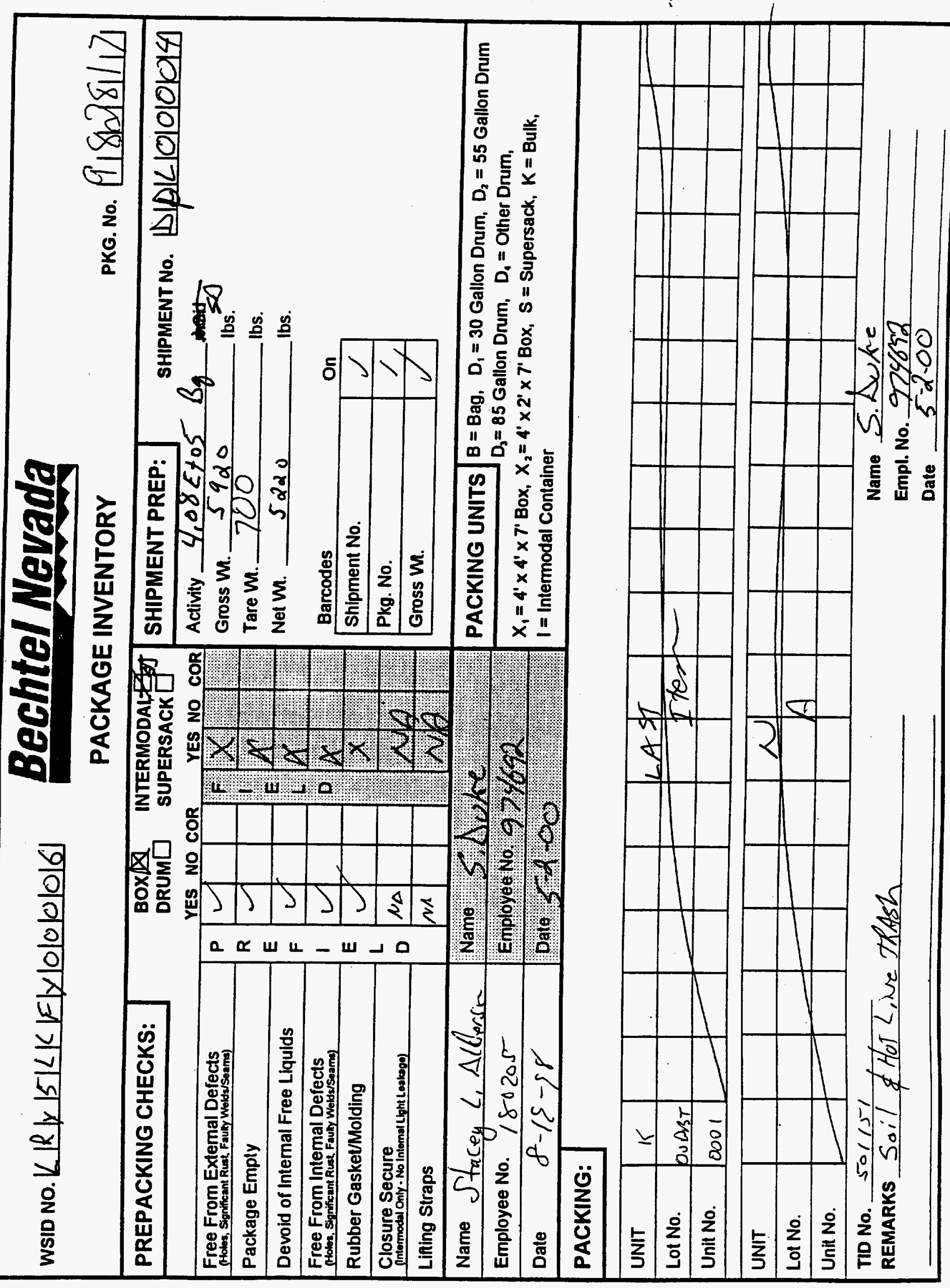




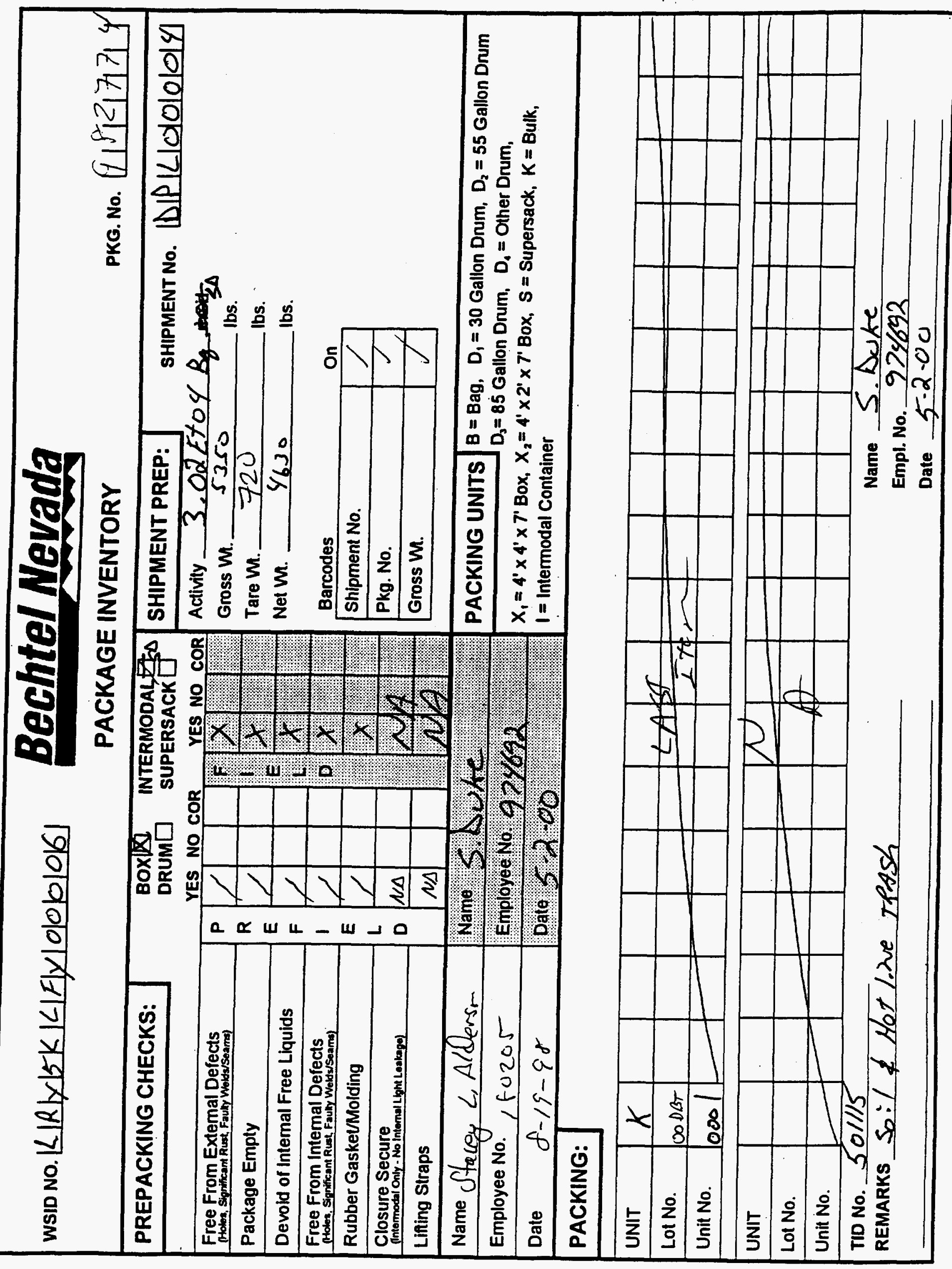




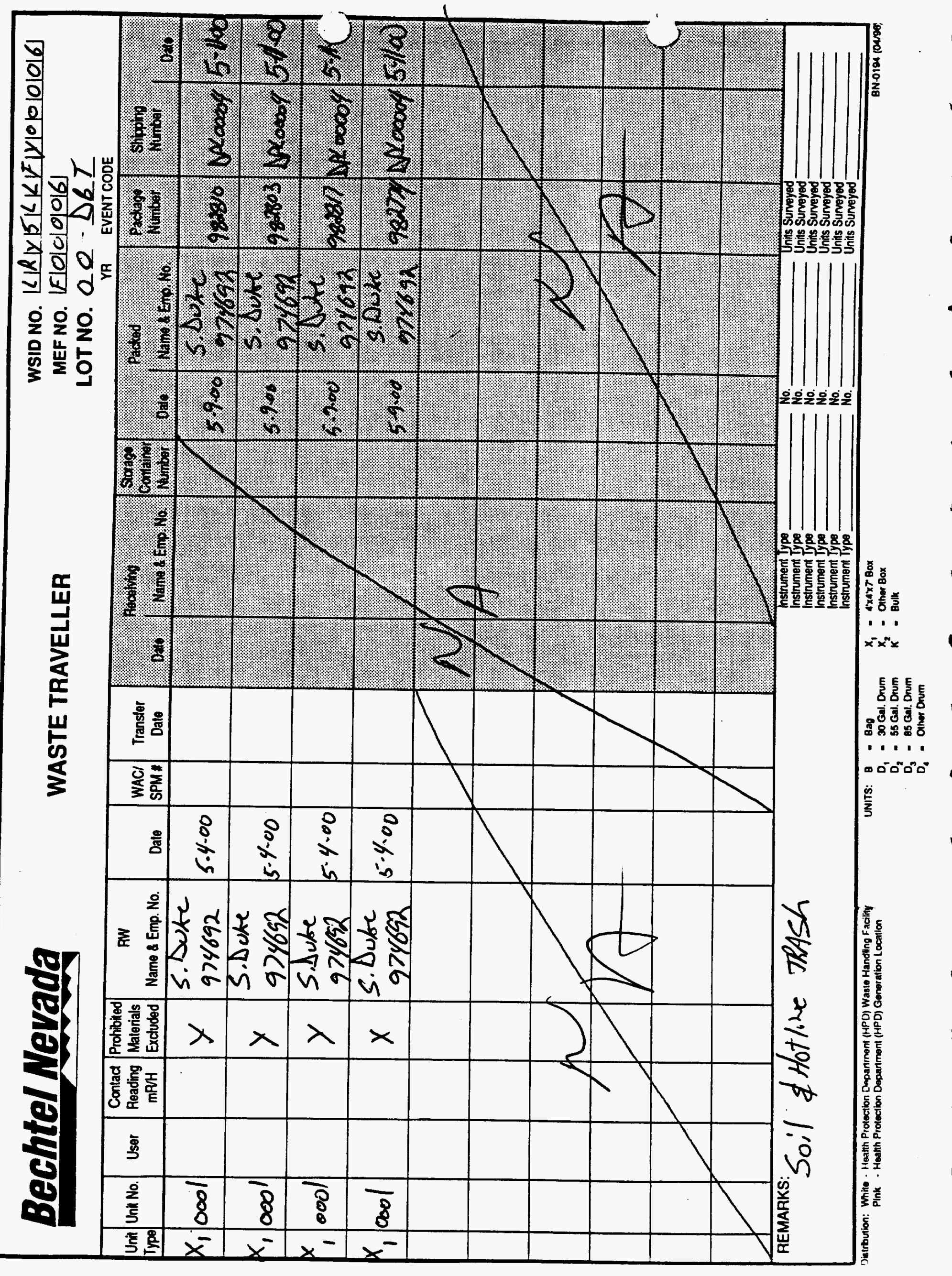




\section{Package Storage and Disposal Request}

Shipment Number. DPL00004

Date: 10-May-00
Prepared By: W ite \& Res.

Manifest Number:

\begin{tabular}{|c|c|c|c|c|c|c|c|c|c|}
\hline \multicolumn{2}{|c|}{$\begin{array}{l}\text { Packege No: } \\
\text { Conteiner Code: } \\
\text { External Volume }\left(m^{\wedge} 3\right) \text { : } \\
\text { Waste Volume }\left(m^{\wedge} 3\right) \text { : } \\
\text { Comment: }\end{array}$} & $\begin{array}{l}292774 \\
210 \\
3):=2.200 E+00 \\
2.034 E+00\end{array}$ & \multicolumn{2}{|c|}{$\begin{array}{l}\text { Contact (mSvm): } \\
\text { I Meter (mSv/h): } \\
\text { Gross Weight (kg): } \\
\text { Net Weight (kg): }\end{array}$} & $\begin{array}{l}0 \\
0 \\
2.427 E+03 \\
2.100 E+03\end{array}$ & $\begin{array}{l}\text { Completed Date: } \\
\text { Operation Type: } \\
\text { Total Activity (bq): } \\
\text { Activity Date: }\end{array}$ & \multicolumn{2}{|c|}{$\begin{array}{l}10+\text { May }-00 \\
B \\
3.019 E+04 \\
09+\text { May }-00\end{array}$} & \\
\hline $\begin{array}{c}\text { Waste Stream } \\
\text { /Profile }\end{array}$ & $\begin{array}{l}\text { Form } \\
\text { Code }\end{array}$ & $\begin{array}{r}F \\
\text { Desec }\end{array}$ & & $\begin{array}{c}\text { Truatment } \\
\text { Code }\end{array}$ & $\begin{array}{l}\text { Treatment } \\
\text { Description }\end{array}$ & $\begin{array}{l}\text { Rev. } \\
\text { No. }\end{array}$ & $\begin{array}{c}\text { Revision } \\
\text { Date }\end{array}$ & Nuclide & $\begin{array}{l}\text { Qty } \\
(\mathrm{Bq})\end{array}$ \\
\hline LRYSLLFYOOAN & 046 & SOL AND HOT & TRASH & 100 & & 00 & $19+$ Apr -00 & AM-241 & $1.560 E+03$ \\
\hline LRYSLLFY0000 & 046 & SOLL AND HOI & TRASH & 100 & & 00 & 10 Apr-00 & $P U-241$ & $8.050 E+03$ \\
\hline LRYSLLFY00006 & 046 & SOIL ANO HOT & TRASH & 100 & & $\infty$ & 19 Apr-00 & PU-240 & $1.780 E+03$ \\
\hline LRY5LLFY00006 & 046 & SOLL AND HOT & TRASH & 100 & & $\infty 0$ & 10 -Apr-00 & PU-239 & $1.880 E+04$ \\
\hline
\end{tabular}

\begin{tabular}{|c|c|c|c|c|c|c|c|c|c|c|}
\hline \multicolumn{2}{|c|}{$\begin{array}{l}\text { Package No: } \\
\text { Container Code: } \\
\text { External Volume }\left(m^{n} 3\right) \text { : } \\
\text { Wacte Volume }\left(m^{n 3}\right) \text { : } \\
\text { Comment: }\end{array}$} & $\begin{array}{l}282003 \\
210 \\
3): \\
2.260 E+\infty 0 \\
2.034 E+\infty 0\end{array}$ & \multicolumn{2}{|c|}{$\begin{array}{l}\text { Contuct (mSv/h): } \\
1 \text { Meter (mSv/h): } \\
\text { Groes Weight (ko): } \\
\text { Net Weight (ko): }\end{array}$} & $\begin{array}{l}0 \\
0 \\
3.406 E+03 \\
3.075 E+03\end{array}$ & \multicolumn{2}{|c|}{$\begin{array}{l}\text { Completed Date: } \\
\text { Operation Type: } \\
\text { Total Activity (bq): } \\
\text { Activity Date: }\end{array}$} & \multicolumn{2}{|c|}{$\begin{array}{l}10-M a y-00 \\
\text { B } \\
3.224 E+04 \\
09-M a y-00\end{array}$} & \\
\hline $\begin{array}{c}\text { Wate Straem } \\
\text { IProfile }\end{array}$ & $\begin{array}{l}\text { Form } \\
\text { Code }\end{array}$ & \multicolumn{2}{|c|}{$\begin{array}{c}\text { Form } \\
\text { Description }\end{array}$} & $\begin{array}{c}\text { Treatment } \\
\text { Code }\end{array}$ & \multicolumn{2}{|c|}{$\begin{array}{l}\text { Treatment } \\
\text { Description }\end{array}$} & $\begin{array}{l}\text { Rev. } \\
\text { No. }\end{array}$ & $\begin{array}{c}\text { Revision } \\
\text { Date }\end{array}$ & Nuclide & $\begin{array}{l}\text { Qty } \\
\text { (Bq) }\end{array}$ \\
\hline LRYGLLFYocoss & 96 & \multicolumn{2}{|c|}{ SOLL AND HOTUNE TRASH } & 100 & & & $\infty$ & 19-Apr-00 & $A M-241$ & $1.980 E+03$ \\
\hline LRYSUFY0000s & 96 & \multicolumn{2}{|c|}{ SOLL AND MOTLNE TRASH } & 100 & & & $\infty$ & $10-\operatorname{Apr}-00$ & PU-241 & $1.020 E+04$ \\
\hline LRYSUFYO0008 & 946 & \multicolumn{2}{|c|}{ SOIL AND HOT UNE TRASH } & 100 & & & $\infty$ & 19-Apr-00 & PU-240 & $2.260 E+03$ \\
\hline LRYSLLFYO0008 & 046 & \multicolumn{2}{|c|}{ SOL AND HOTLNE TRASH } & 100 & & & $\infty$ & 19-Apr-00 & PU-239 & $2.380 E+04$ \\
\hline \multicolumn{2}{|c|}{$\begin{array}{l}\text { Conteiner Cods: } \\
\text { Extemal Volume (m^3 } \\
\text { Weate Volume }\left(m^{\wedge} 3\right) \text { : }\end{array}$} & $\begin{array}{l}252810 \\
210 \\
3):=2.260 E+00 \\
2.034 E+00\end{array}$ & $\begin{array}{l}\text { Contact } \\
1 \text { Meter } \\
\text { Groes V } \\
\text { Net We }\end{array}$ & $\begin{array}{l}\text { nSv/n): } \\
\text { nSv/n): } \\
\text { sight (ko): } \\
\text { ht (ko): }\end{array}$ & $\begin{array}{l}0 \\
0 \\
3.590 E+03 \\
3.261 E+03\end{array}$ & & $\begin{array}{l}\text { Dete: } \\
\text { Type: } \\
\text { y (bq): }\end{array}$ & $\begin{array}{l}10-\text { May } \\
\text { B } \\
3.343 E \\
09-\text { May }\end{array}$ & & \\
\hline $\begin{array}{c}\text { Wacte Stream } \\
\text { Profita }\end{array}$ & $\begin{array}{l}\text { Form } \\
\text { Code }\end{array}$ & Fe & & $\begin{array}{c}\text { Treodment } \\
\text { Code }\end{array}$ & & & $\begin{array}{l}\text { Rev. } \\
\text { No. }\end{array}$ & $\begin{array}{c}\text { Revieion } \\
\text { Date }\end{array}$ & Nuclide & $\begin{array}{l}\text { Oty } \\
\text { (Bq) }\end{array}$ \\
\hline LRYJUFYOCOOS & $\omega$ & SOL AND HOT & TRASH & 100 & & & 00 & $19-4 p r-10$ & AM-241 & $1.730 E+06$ \\
\hline LRYBLFY00006 & 24 & SOIL AND HOT & TRASH & 100 & & & $\infty$ & $19-\operatorname{Apr}-00$ & PU-2A1 & $8.820 E+06$ \\
\hline LRYSUFY00008 & 046 & SOIL AND HOT & TRASH & 100 & & & $\mathbf{\infty 0}$ & 19 Apr-A0 & PU-240 & $1.980 E+06$ \\
\hline LRYSLLFYO0006 & 046 & SOIL AND HOT & TRASH & 100 & & & $\infty$ & $18-A p r-00$ & PU-239 & $2.080 E+07$ \\
\hline
\end{tabular}




\section{Package Storage and Disposal Request}

Shipment Number: DPL00004

Prepared By:

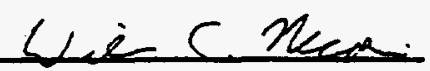

Date: 10-May-00

Manifest Number:

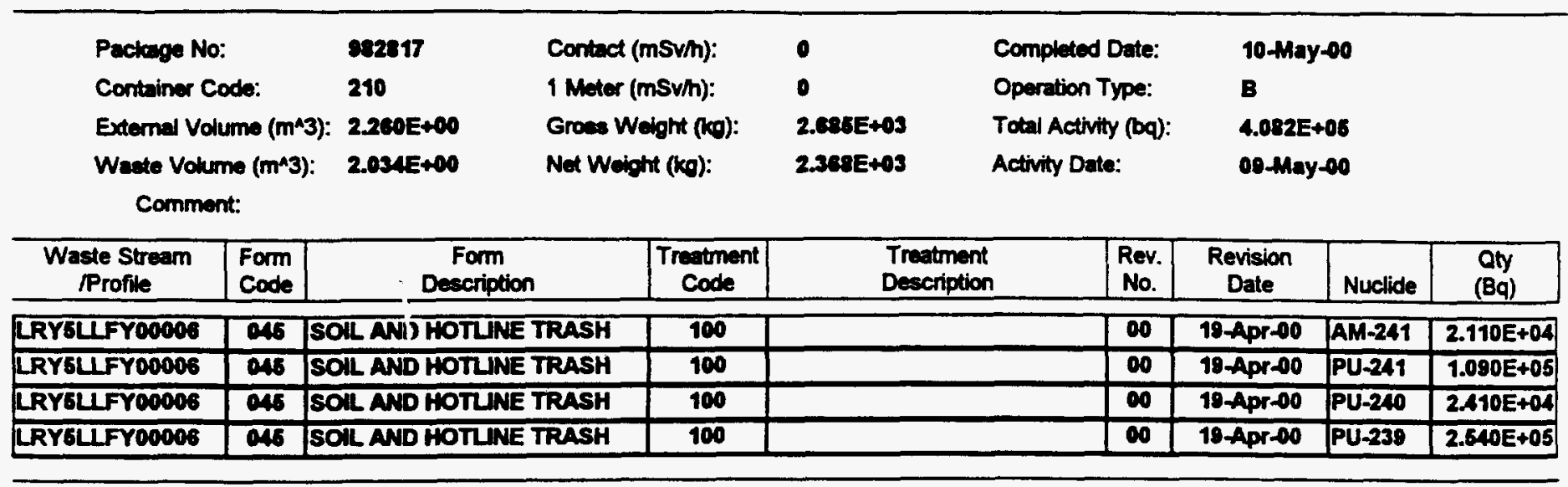





\section{APPENDIX C}

\section{VERIFICATION SAMPLE ANALYTICAL REPORTS}





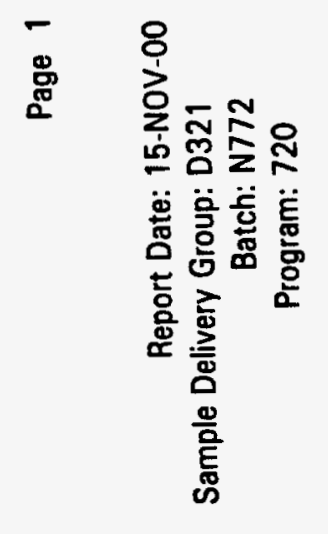

\begin{tabular}{|c|c|c|c|c|c|c|c|c|c|c|}
\hline 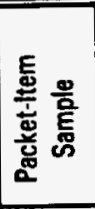 & 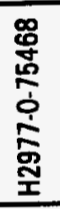 & 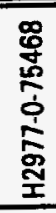 & 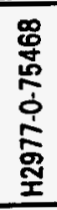 & 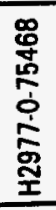 & 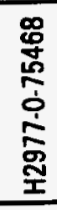 & 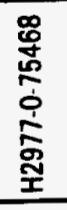 & 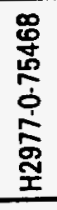 & 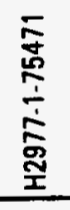 & 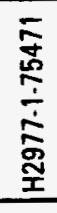 & 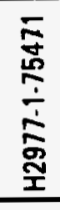 \\
\hline 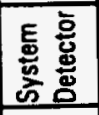 & $\begin{array}{l}\overline{1} \\
\text { 웅 }\end{array}$ & $\begin{array}{l}\overline{0} \\
\text { ' }\end{array}$ & 훙 & $\begin{array}{l}\overline{0} \\
\text { 宫 }\end{array}$ & $\begin{array}{l}\overline{0} \\
\text { 영 }\end{array}$ & $\begin{array}{l}\overline{9} \\
\text { 영 }\end{array}$ & $\begin{array}{l}\overline{0} \\
\text { 용 }\end{array}$ & $\begin{array}{l}\bar{\delta} \\
\text { : }\end{array}$ & $\begin{array}{l}\bar{\delta} \\
\text { ஸे }\end{array}$ & $\begin{array}{l}\bar{\sigma} \\
\text { 잉 }\end{array}$ \\
\hline 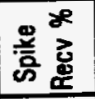 & & & & & & & & & & \\
\hline 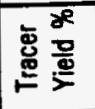 & & & & & & & & & & \\
\hline 胥受 & $\mathbf{E}$ & $\mathbf{E}$ & E & $\mathbf{E}$ & E & E & $\Xi$ & E & $\mathbf{E}$ & $\mathrm{E}_{\mathrm{\sigma}}$ \\
\hline
\end{tabular}

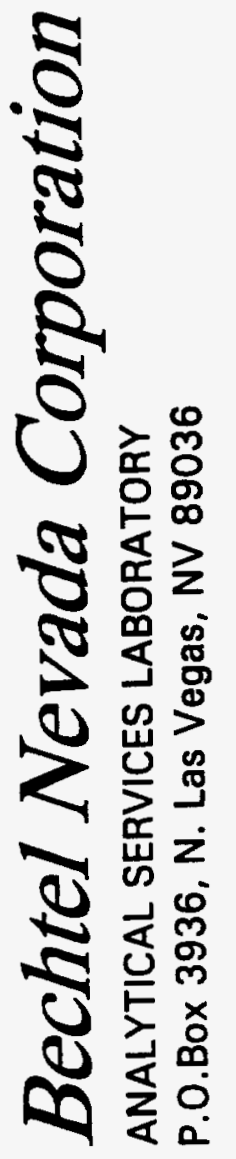

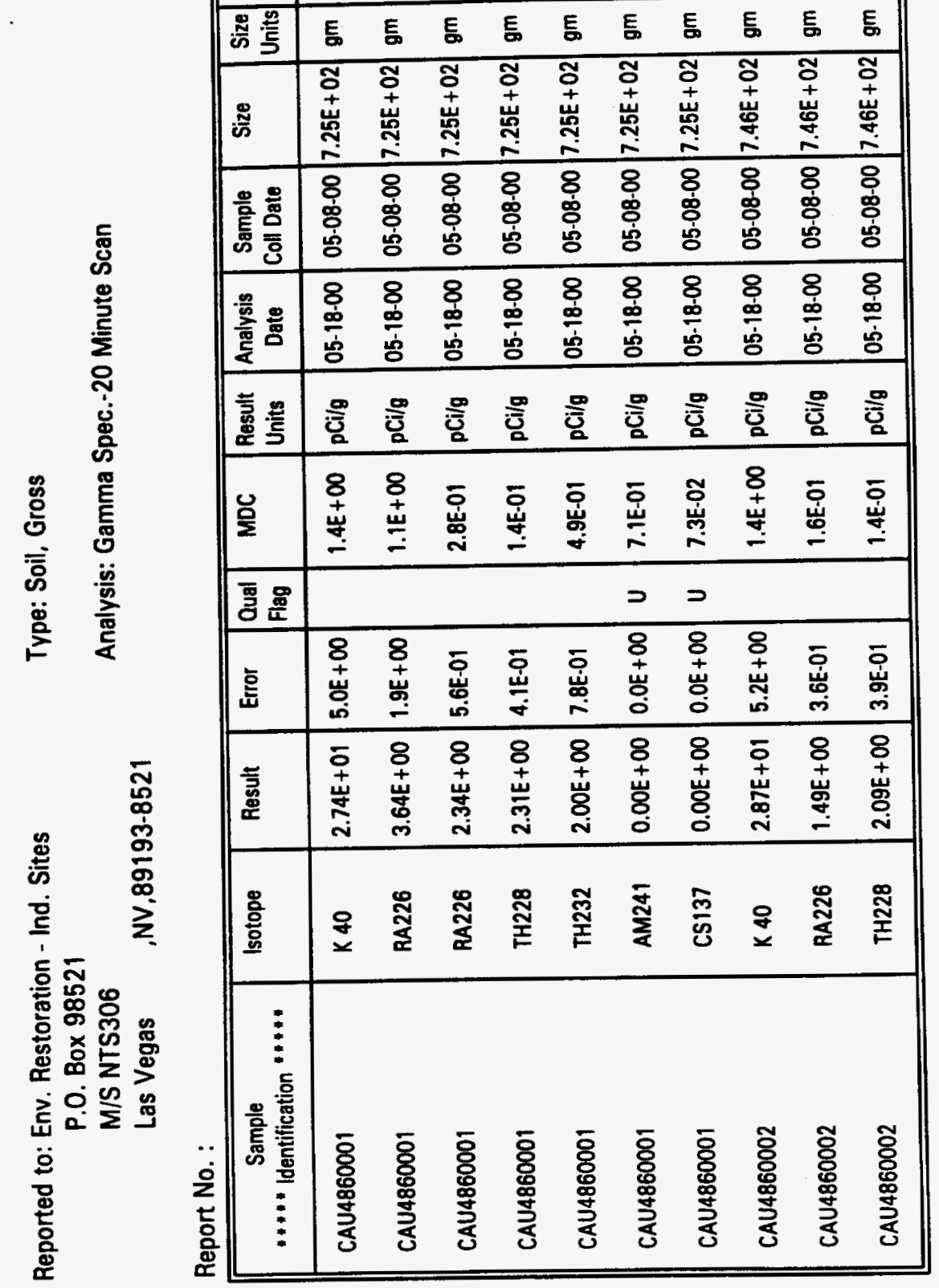

$\stackrel{\ddot{0}}{2}$

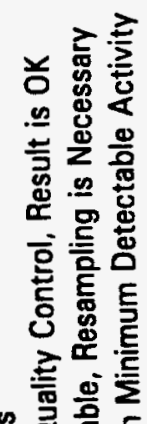

흘음

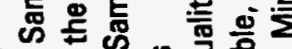

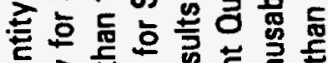

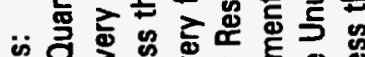

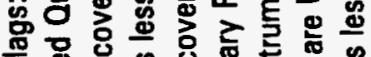

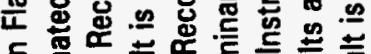
들 들

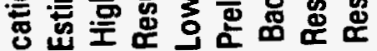

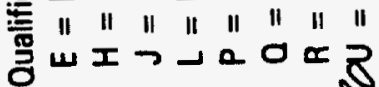

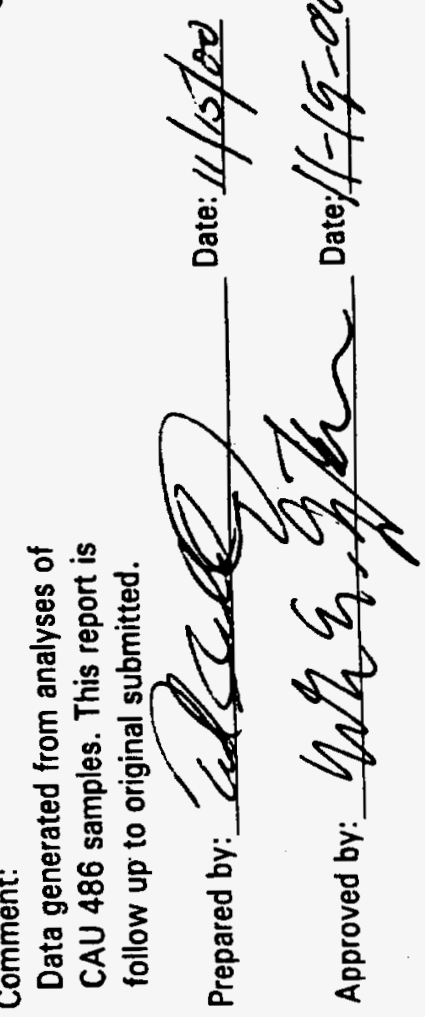




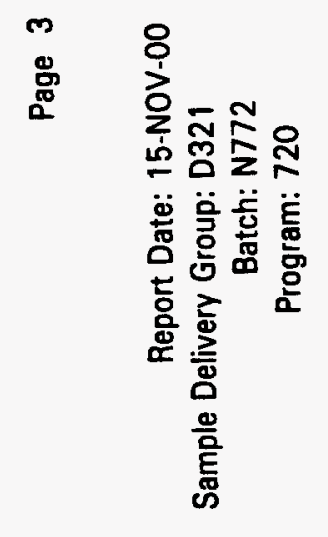

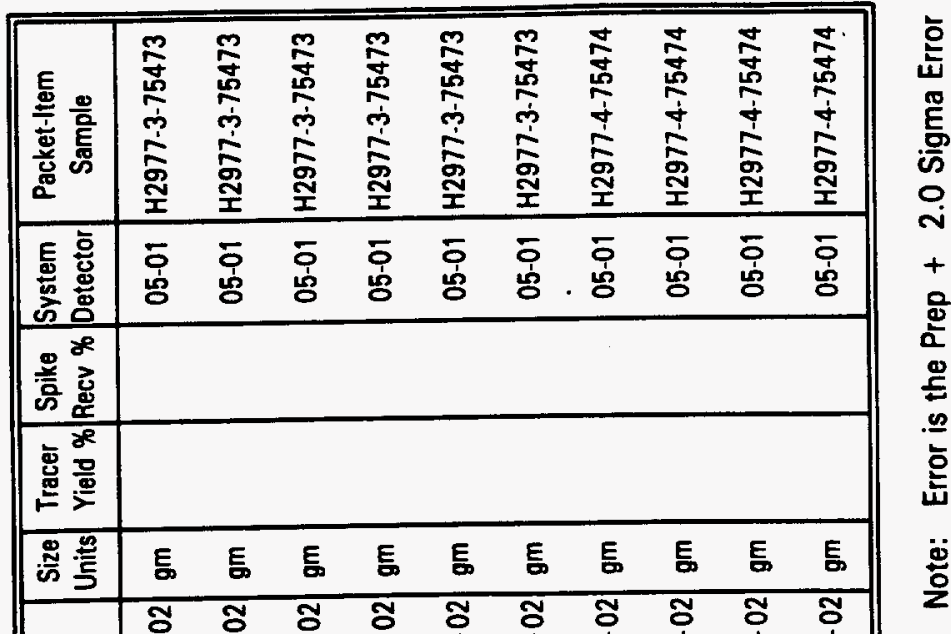
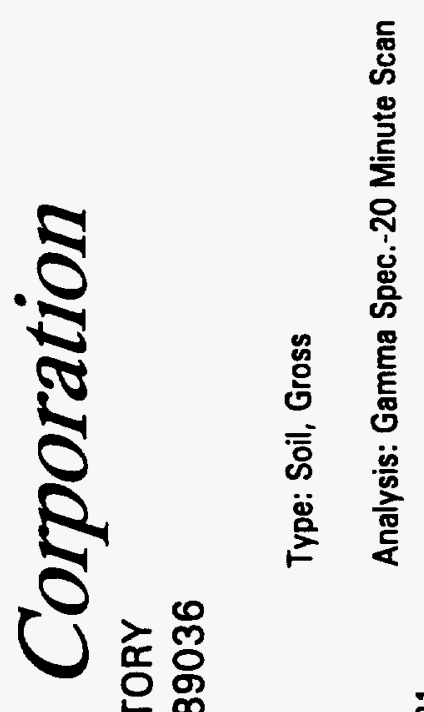

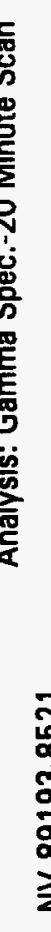

$\sum$ Uु

(U)

To

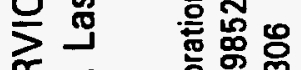

䍃 2

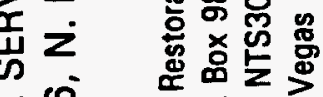

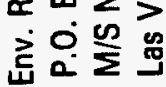

त 8

는

仓 $\ddot{2}$ 产
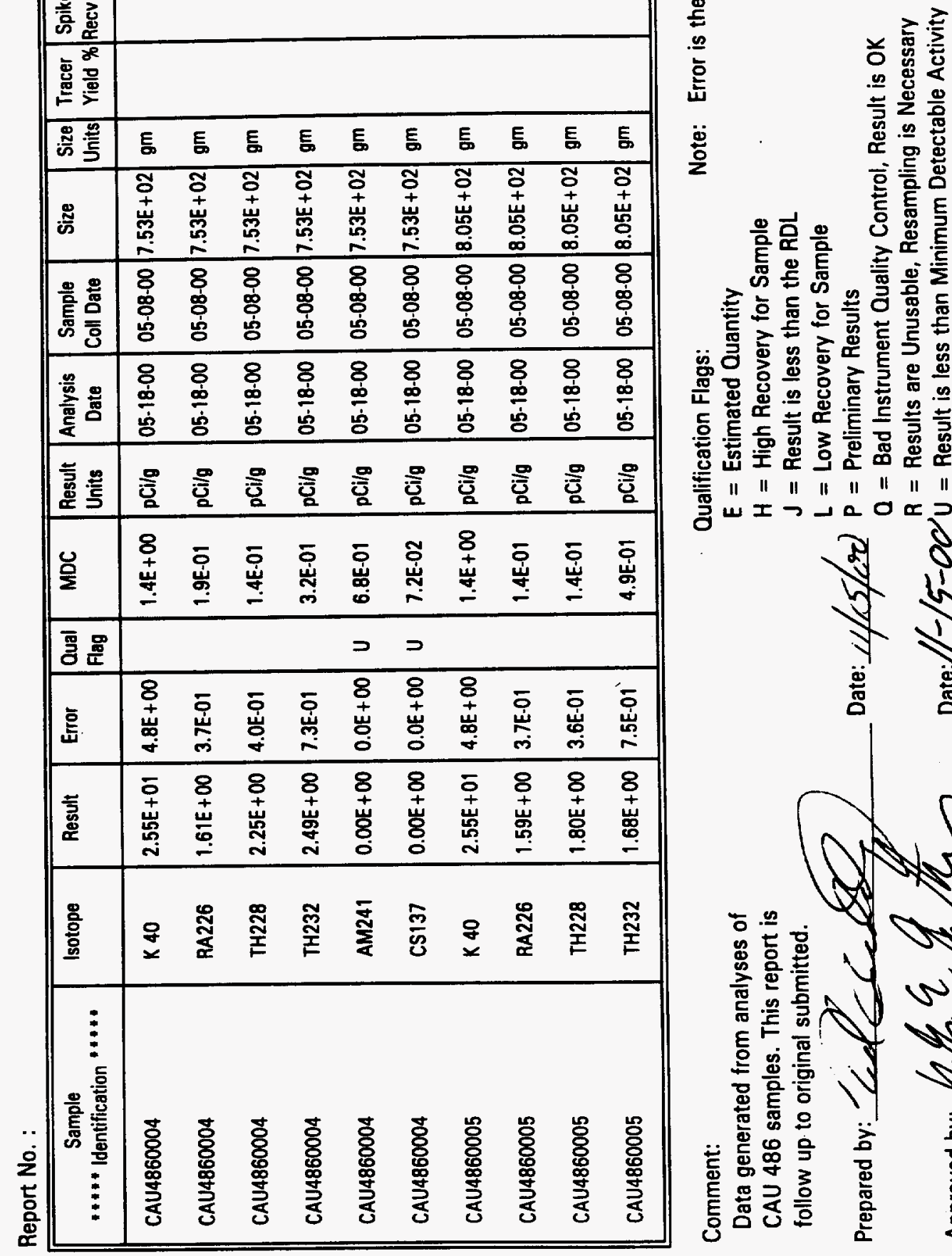

음뭉

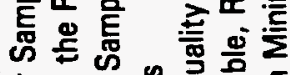

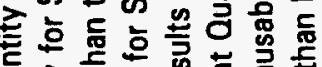

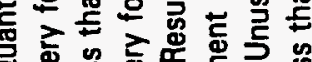

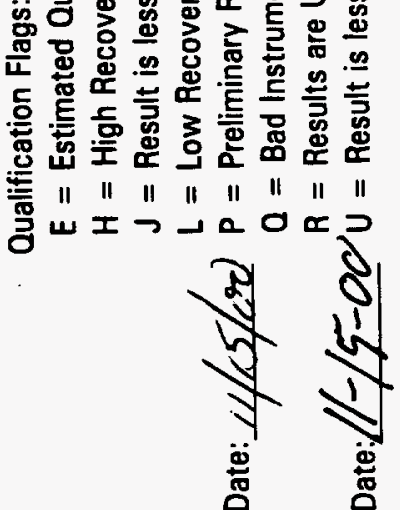

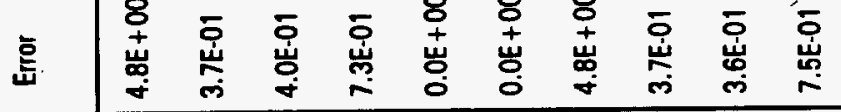

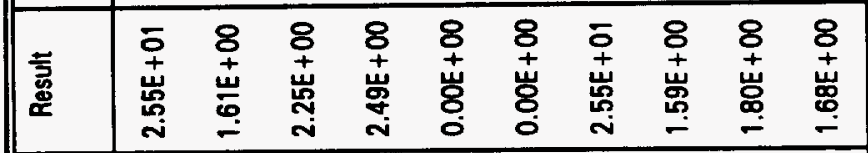

\begin{tabular}{|c|c|c|c|c|c|c|c|c|c|c|}
\hline 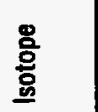 & $\underset{x}{g}$ & 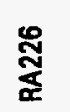 & 昰 & 忍 & $\underset{\frac{\tilde{Z}}{\alpha}}{\bar{z}}$ & $\frac{\widehat{9}}{50}$ & $\underset{*}{q}$ & స్స్ & 莡 & 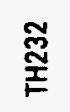 \\
\hline 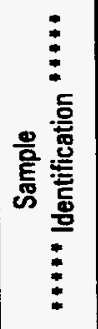 & 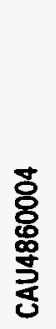 & 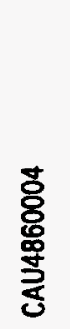 & 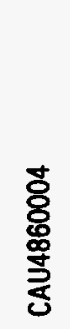 & 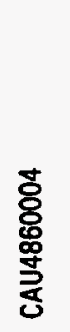 & 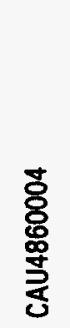 & 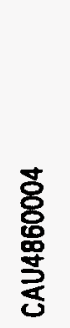 & 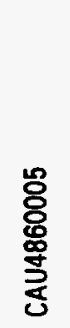 & 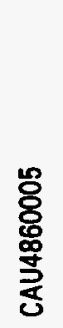 & 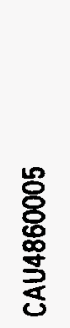 & 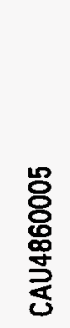 \\
\hline
\end{tabular}




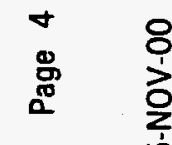

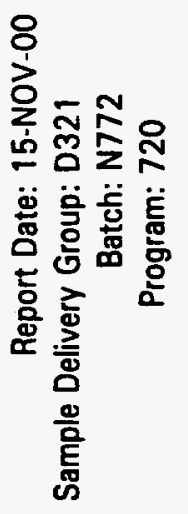

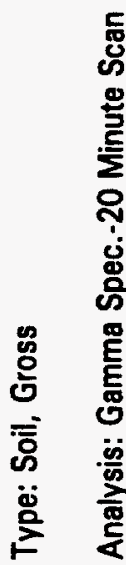

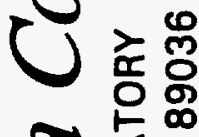

$\circlearrowleft \frac{\pi}{4}$

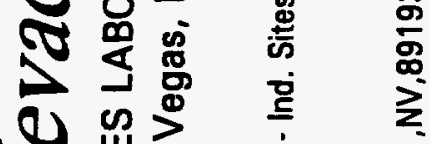

(1)

$\sum \sum^{U}$

䍃之

U 山

U J

U

(1) $\frac{1}{2}$

$\infty \sum_{0}$

드ำ

可

\begin{tabular}{|c|c|c|c|c|c|c|c|c|c|c|}
\hline 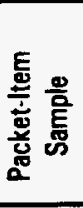 & 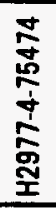 & 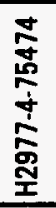 & 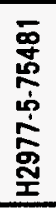 & 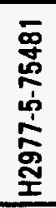 & 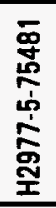 & 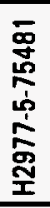 & 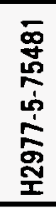 & 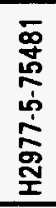 & 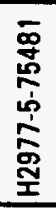 & 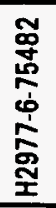 \\
\hline 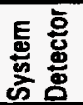 & $\begin{array}{l}\overline{0} \\
\text { 잉 }\end{array}$ & $\begin{array}{l}\overline{1} \\
\text { 용 }\end{array}$ & $\begin{array}{l}\overline{0} \\
\text { 잉 }\end{array}$ & $\begin{array}{l}\text { 훙 } \\
\text { هos }\end{array}$ & $\begin{array}{l}\bar{\delta} \\
\text { 宫 }\end{array}$ & $\begin{array}{l}\bar{\delta} \\
\text { !े }\end{array}$ & $\begin{array}{l}\bar{\delta} \\
\text { d. }\end{array}$ & $\begin{array}{l}\overline{0} \\
\text { 잉 }\end{array}$ & $\begin{array}{l}\overline{0} \\
\text { ㅇㅇㅇ }\end{array}$ & $\begin{array}{l}\overline{0} \\
\text { 용 }\end{array}$ \\
\hline 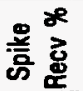 & & & & & & & & & & \\
\hline 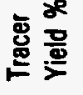 & & & & & & & & & & \\
\hline ऊั้ & E & E & E & E & $\mathbf{E}$ & క్ & E & $\mathbf{E}_{\mathrm{S}}$ & 巨్ & E \\
\hline & శ్ & గ్ & శ్ & $\bar{\sigma}$ & ธี & ธี & ชี & శ్ & శ & $\tilde{O}$ \\
\hline
\end{tabular}

ذ్

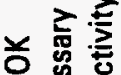

is

至

号

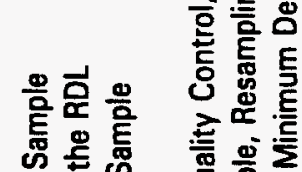

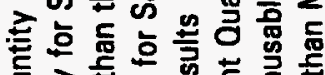

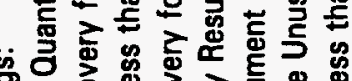

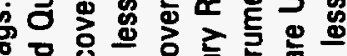

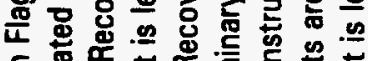
든 눈

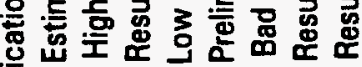
")

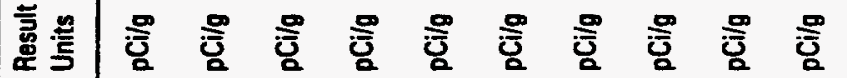

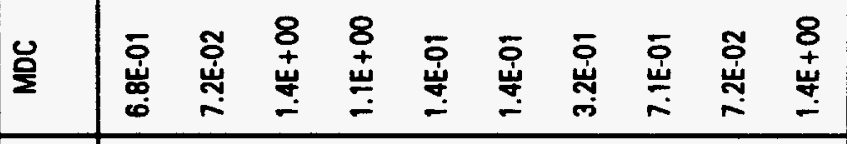

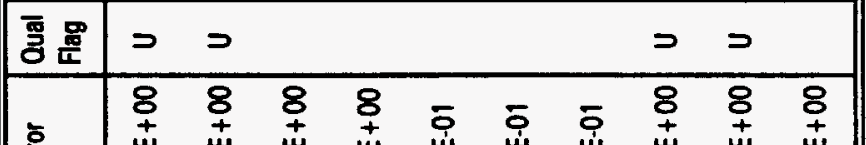

\begin{tabular}{|c|c|c|c|c|c|c|c|c|c|}
\hline & $\begin{array}{l}8 \\
+ \\
\text { 号 } \\
0\end{array}$ & $\begin{array}{l}+ \\
+ \\
\text { + } \\
0\end{array}$ & $\begin{array}{l}\stackrel{+}{+} \\
\stackrel{+}{\underset{*}{*}}\end{array}$ & 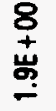 & 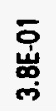 & $\begin{array}{l}\bar{D} \\
\dot{U} \\
\dot{U}\end{array}$ & & $\begin{array}{l}\mathbf{0} \\
\stackrel{+}{0} \\
0 \\
0\end{array}$ & $\begin{array}{l}\text { + } \\
\text { 㟧 } \\
\end{array}$ \\
\hline 5 & 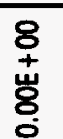 & $\begin{array}{l}8 \\
+ \\
\text { 岁 } \\
0\end{array}$ & 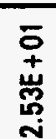 & 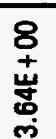 & $\begin{array}{l}8 \\
+ \\
\mathbf{w} \\
0\end{array}$ & 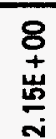 & 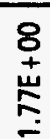 & $\begin{array}{l}8 \\
+ \\
\text { 山्. } \\
0\end{array}$ & 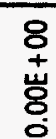 \\
\hline
\end{tabular}

产

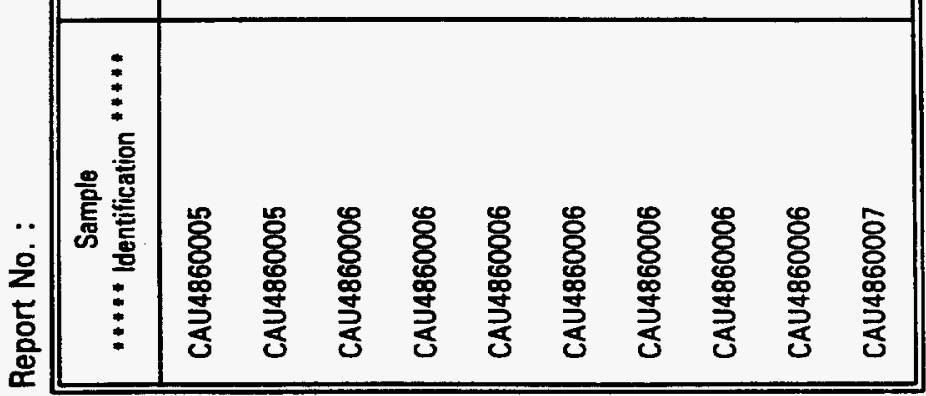

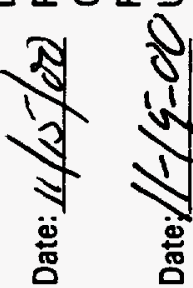

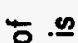

岁 능

른

들

은

흠

兽 劳

这密怘

兽还 


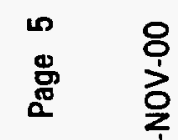

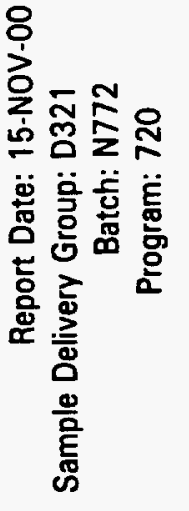

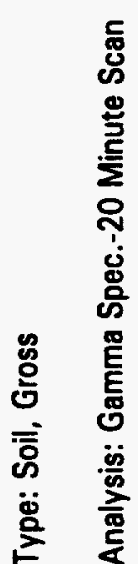

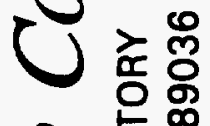

$8 \stackrel{5}{4}$

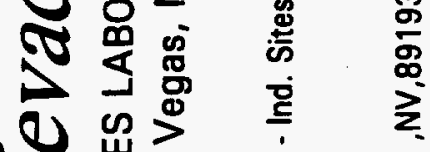

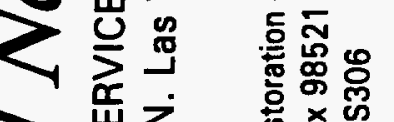

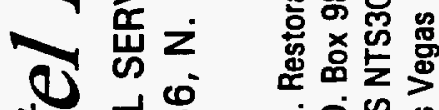

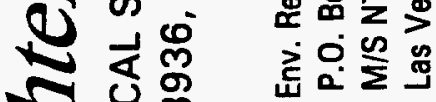

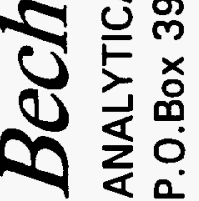

㤩

\begin{tabular}{|c|c|c|c|c|c|c|c|c|c|c|}
\hline 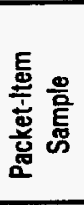 & 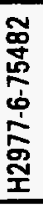 & 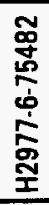 & 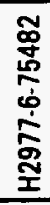 & 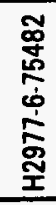 & 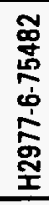 & 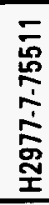 & 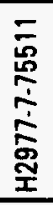 & 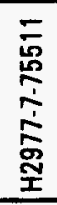 & 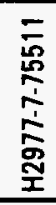 & 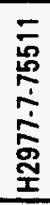 \\
\hline 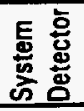 & $\begin{array}{l}\bar{\delta} \\
\text { is } \\
1\end{array}$ & $\begin{array}{l}\overline{0} \\
\text { ㅇㅇㅇ }\end{array}$ & $\begin{array}{l}\overline{0} \\
\dot{1} \\
\text { b }\end{array}$ & $\begin{array}{l}\overline{0} \\
\text { di }\end{array}$ & $\begin{array}{l}\bar{D} \\
\text { iे }\end{array}$ & $\begin{array}{l}\overline{0} \\
\text { 's }\end{array}$ & $\begin{array}{l}\text { 웅 } \\
\text { 's }\end{array}$ & $\begin{array}{l}\overline{0} \\
\text { i⿱宀 }\end{array}$ & $\begin{array}{l}\bar{\delta} \\
\text { : }\end{array}$ & $\begin{array}{l}\bar{\delta} \\
\text { 옹 }\end{array}$ \\
\hline 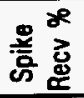 & & & & & & & & & & \\
\hline 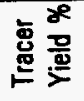 & & & & & & & & & & \\
\hline 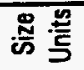 & E & E & E⿸\zh14口 & E & E & 㤩 & E & 畐 & 㤩 & E \\
\hline
\end{tabular}

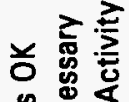

으 웅

苛离

동

醇

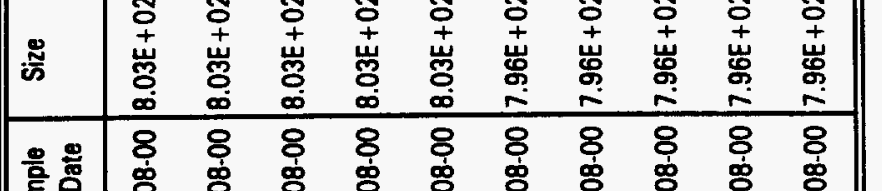

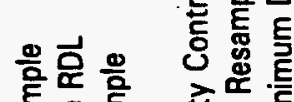

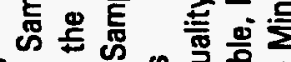

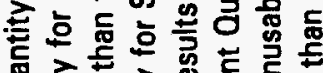

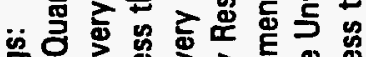
泵

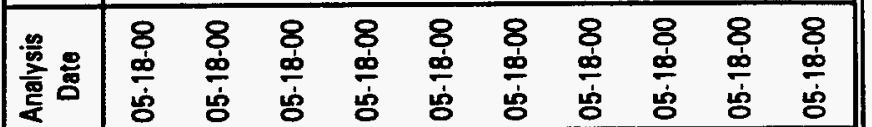

产

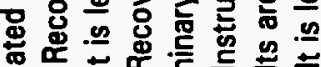
둔

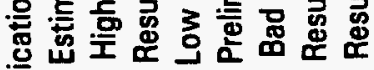
"

\begin{tabular}{|c|c|c|c|c|c|c|c|c|c|c|}
\hline 일 & $\begin{array}{l}\overline{0} \\
\text { 岁 }\end{array}$ & 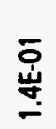 & $\begin{array}{l}\bar{\delta} \\
\text { 岁 } \\
\text { ơ }\end{array}$ & $\begin{array}{l}\bar{D} \\
\text { 岁 } \\
\text { do }\end{array}$ & 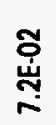 & 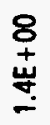 & $\begin{array}{l}\bar{D} \\
\text { 岁 }\end{array}$ & & $\begin{array}{l}\bar{\delta} \\
\text { 岁 } \\
\dot{v}\end{array}$ & $\begin{array}{l}\bar{D} \\
\text { 岁 } \\
\text { b }\end{array}$ \\
\hline
\end{tabular}
子

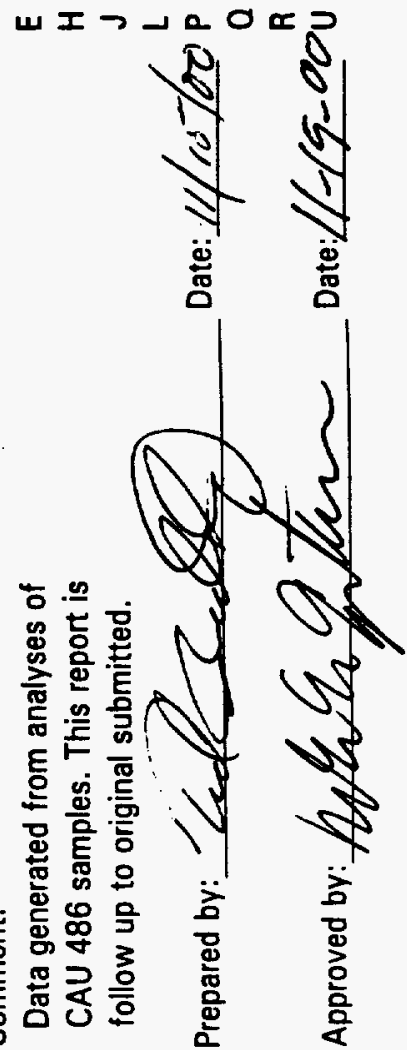




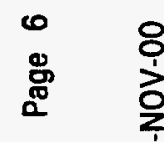

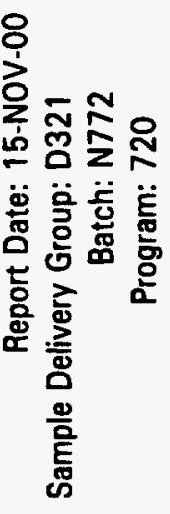

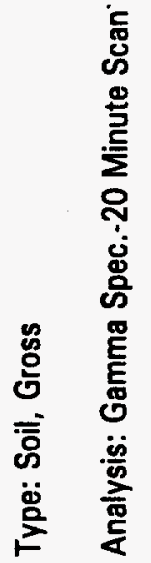

¿ $\stackrel{0}{\circ}$ 요

용

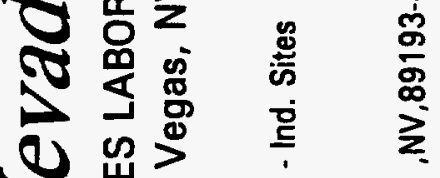
山 는 든

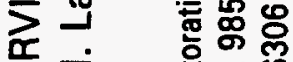
㟧之新号 山 巡灾 न ठु

C

U

a $\sum_{<} 0$

\begin{tabular}{|c|c|c|c|c|c|}
\hline 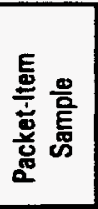 & 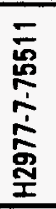 & 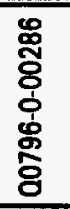 & 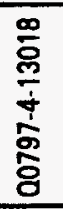 & 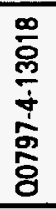 & $\begin{array}{l}\frac{\infty}{0} \\
\frac{0}{+} \\
\frac{5}{0} \\
\overline{8}\end{array}$ \\
\hline 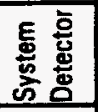 & $\begin{array}{l}\bar{\phi} \\
\text { ì }\end{array}$ & $\begin{array}{l}\overline{0} \\
\text { 잉 }\end{array}$ & $\begin{array}{l}\bar{\gamma} \\
\text { 广் }\end{array}$ & $\begin{array}{l}\bar{\alpha} \\
\text { 형 }\end{array}$ & $\begin{array}{l}\bar{\sigma} \\
\text { id }\end{array}$ \\
\hline $\begin{array}{l}\Phi^{*} \\
\text { 竞 } \\
\end{array}$ & & & में & $\frac{7}{5}$ & $\overline{\bar{g}}$ \\
\hline 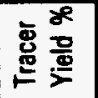 & & & & & \\
\hline 总产 & E & 㤩 & $\mathbf{E}$ & $\mathbf{E}$ & $\underline{\mathbf{E}}$ \\
\hline జั้ & 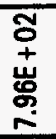 & $\begin{array}{l}8 \\
+ \\
\stackrel{山}{8} \\
\\
\end{array}$ & 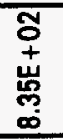 & 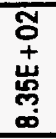 & 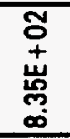 \\
\hline $\begin{array}{l}\text { 응 } \\
\text { 总 } \\
\text { 心 }\end{array}$ & $\begin{array}{l}8 \\
8 \\
0 \\
01 \\
\text { iो }\end{array}$ & $\begin{array}{l}\frac{8}{0} \\
\\
\\
\end{array}$ & 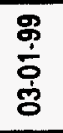 & 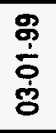 & 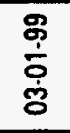 \\
\hline 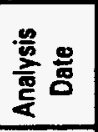 & $\begin{array}{l}8 \\
\dot{\phi} \\
\dot{\phi} \\
\dot{\phi}\end{array}$ & $\begin{array}{l}\stackrel{8}{\dot{\phi}} \\
\dot{\phi} \\
\dot{\phi}\end{array}$ & $\begin{array}{l}8 \\
\dot{\infty} \\
\dot{\phi} \\
\dot{8}\end{array}$ & 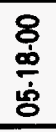 & 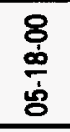 \\
\hline 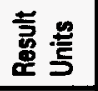 & 혐 & 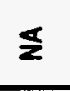 & 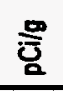 & 홍 & 兽 \\
\hline ஜू & 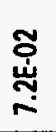 & $\begin{array}{l}8 \\
+ \\
+ \\
0 \\
0\end{array}$ & 亯 & 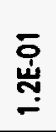 & $\begin{array}{l}\widetilde{S} \\
\text { 岕 } \\
\sigma\end{array}$ \\
\hline 覀嵒 & $>$ & D & & & \\
\hline ఏ్ & $\begin{array}{l}8 \\
+ \\
4 \\
0\end{array}$ & $\begin{array}{l}8 \\
+ \\
\pm \\
0 \\
\end{array}$ & $\begin{array}{l}8 \\
\\
\stackrel{+}{ \pm} \\
\text { i }\end{array}$ & $\begin{array}{l}8 \\
+ \\
\stackrel{+}{ \pm} \\
\text { in }\end{array}$ & 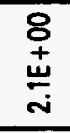 \\
\hline $\begin{array}{l}\text { 节 } \\
\text { 总 }\end{array}$ & $\begin{array}{l}8 \\
+ \\
+ \\
\text { 岁 } \\
0\end{array}$ & $\begin{array}{l}8 \\
+ \\
\text { 山े } \\
\end{array}$ & $\begin{array}{l}\overline{0} \\
+ \\
\stackrel{\tilde{g}}{g} \\
\end{array}$ & $\begin{array}{l}\overline{0} \\
+ \\
\text { 岁 } \\
\text { i }\end{array}$ & $\begin{array}{l}\overline{0} \\
+ \\
\text { 岁 } \\
-\end{array}$ \\
\hline $\begin{array}{l}\text { 음 } \\
\text { 을 }\end{array}$ & $\frac{\bar{m}}{\frac{m}{3}}$ & $\begin{array}{l}\text { 志 } \\
\frac{\mathrm{O}}{\mathrm{U}} \\
\frac{\mathbf{z}}{2} \\
\frac{0}{2}\end{array}$ & 离 & ষ్ర & $\frac{5}{3}$ \\
\hline 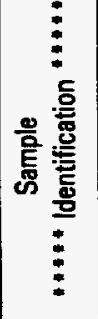 & 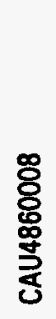 & 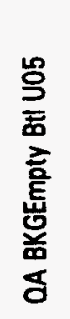 & 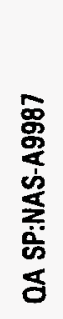 & 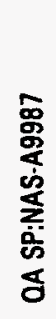 & 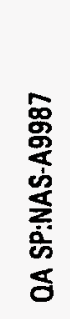 \\
\hline
\end{tabular}

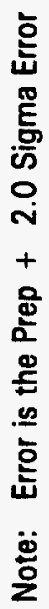

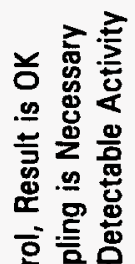

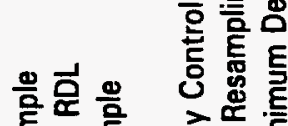

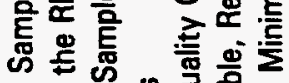

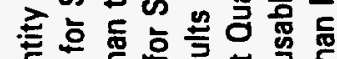

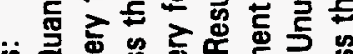

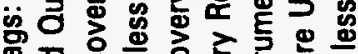

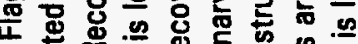

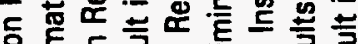

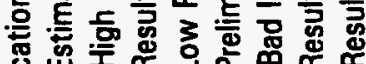
"| | | | " | " |

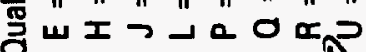

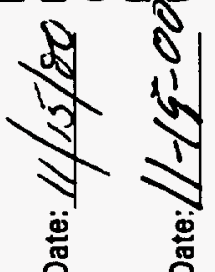

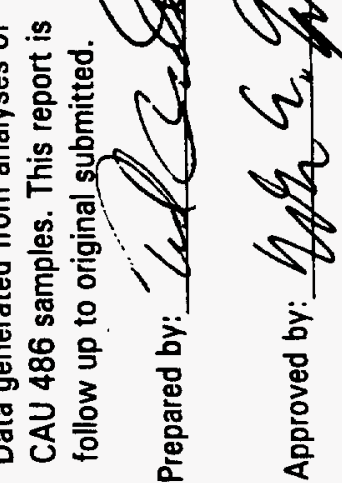




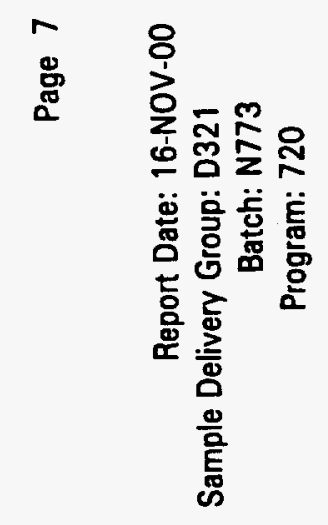

\begin{tabular}{|c|c|c|c|c|c|c|c|c|c|c|}
\hline 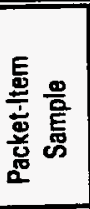 & 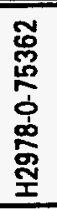 & 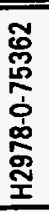 & 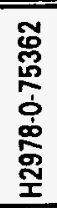 & 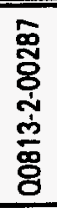 & 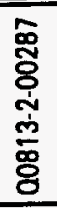 & 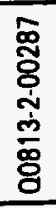 & 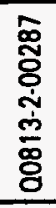 & $\begin{array}{l}\frac{0}{0} \\
\frac{0}{1} \\
\frac{1}{0} \\
\frac{8}{8}\end{array}$ & 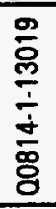 & $\begin{array}{l}\frac{0}{5} \\
\frac{0}{1} \\
\frac{\dot{0}}{80} \\
\end{array}$ \\
\hline 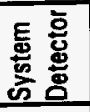 & $\begin{array}{l}\bar{\phi} \\
\dot{8}\end{array}$ & $\begin{array}{l}\overline{0} \\
\text { : }\end{array}$ & $\begin{array}{l}\overline{8} \\
\text { ष̊ }\end{array}$ & $\begin{array}{l}\overline{0} \\
\dot{\phi}\end{array}$ & $\begin{array}{l}\overline{0} \\
\text { ष्ठ }\end{array}$ & $\begin{array}{l}\overline{\dot{\theta}} \\
\text { ष्ठ }\end{array}$ & $\begin{array}{l}\overline{0} \\
\dot{8}\end{array}$ & $\begin{array}{l}\overline{\$} \\
\dot{8} \\
\dot{8}\end{array}$ & $\begin{array}{l}\bar{\phi} \\
\dot{8}\end{array}$ & $\begin{array}{l}\bar{\sigma} \\
\text { ò }\end{array}$ \\
\hline 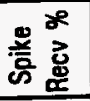 & & & & & & & & $\begin{array}{l}\infty \\
\varnothing \\
\varnothing\end{array}$ & $\stackrel{\mathscr{\sigma}}{\sigma}$ & $\stackrel{m}{\infty}$ \\
\hline 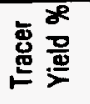 & & & & & & & & & & \\
\hline 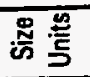 & $\bar{E}$ & $\bar{E}$ & $\overline{\boldsymbol{E}}$ & $\bar{E}$ & $\bar{E}$ & $\bar{E}$ & $\bar{E}$ & $\bar{\varepsilon}$ & $\bar{E}$ & $\bar{E}$ \\
\hline
\end{tabular}
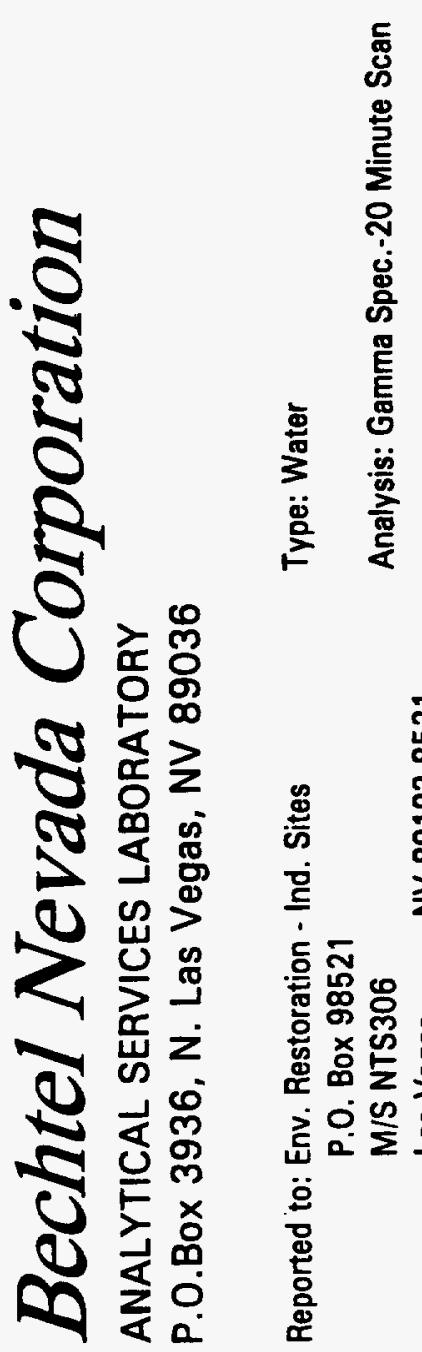

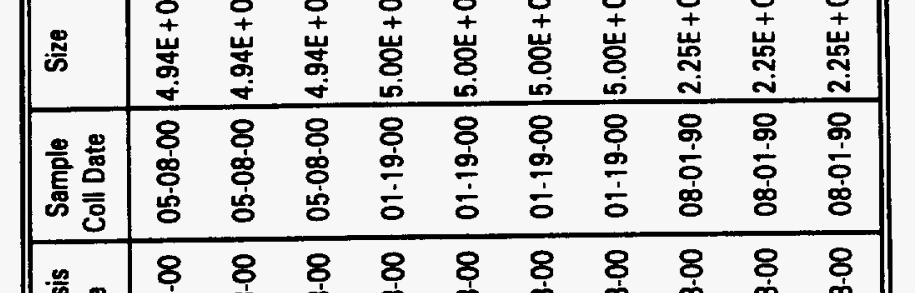



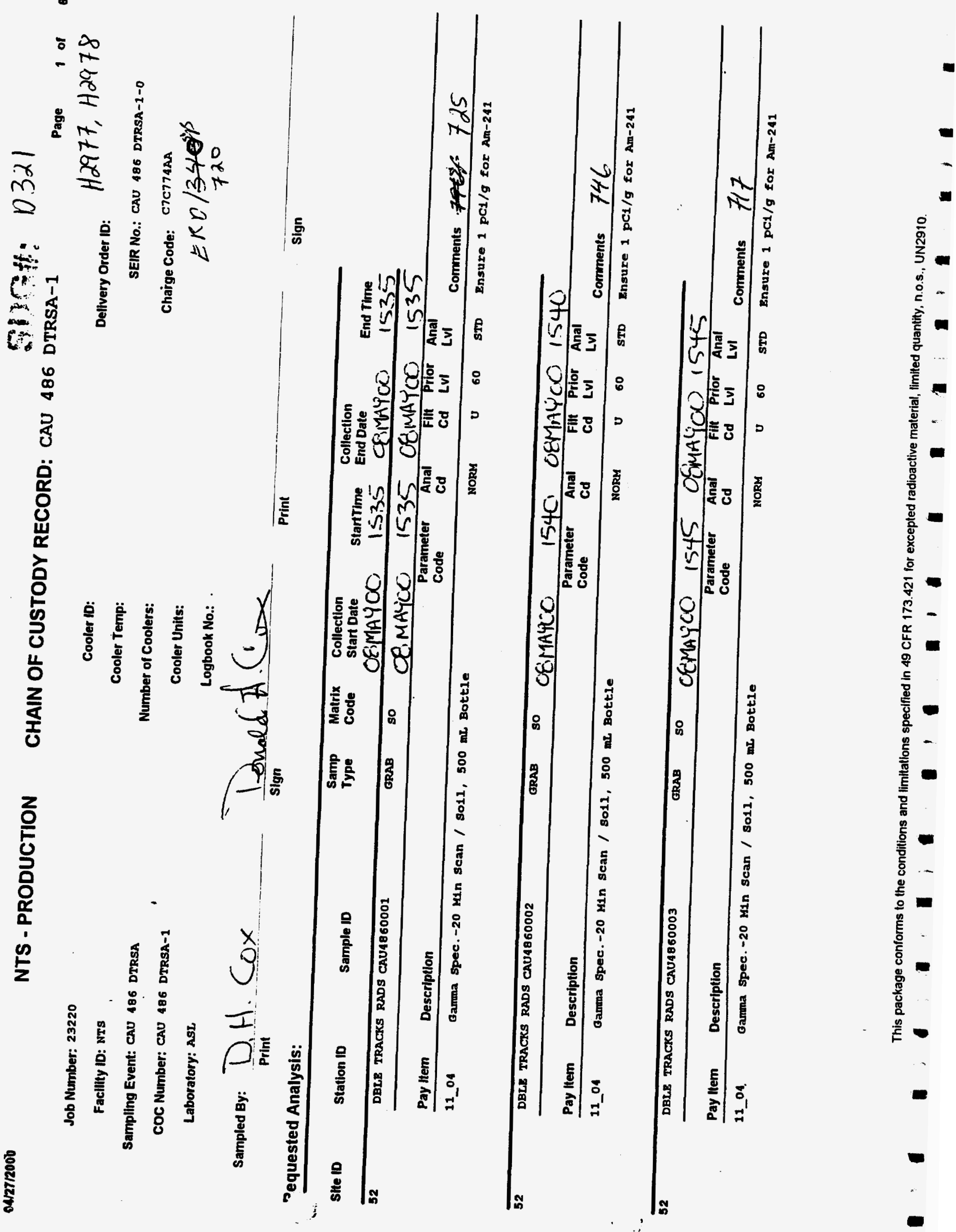


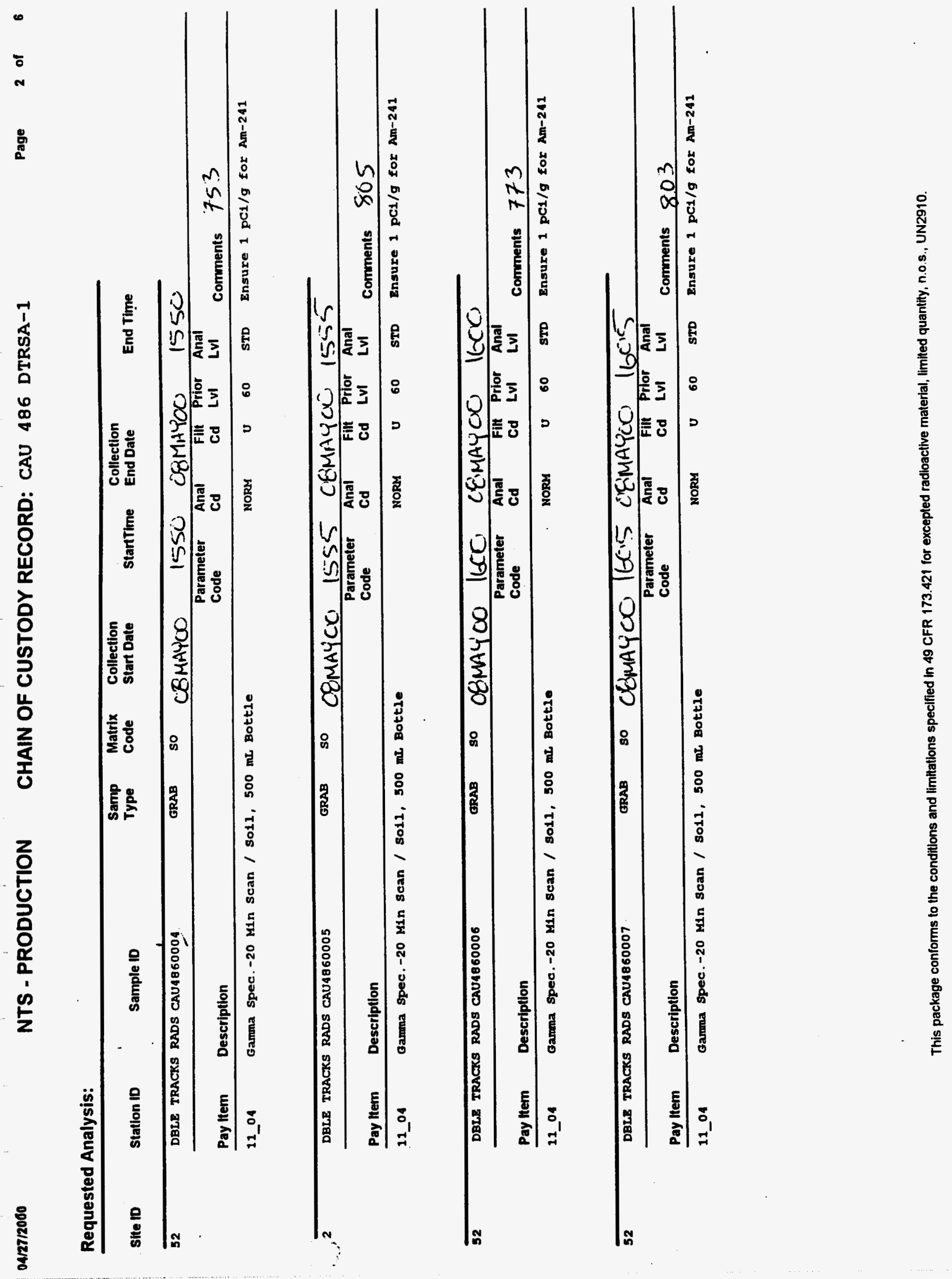




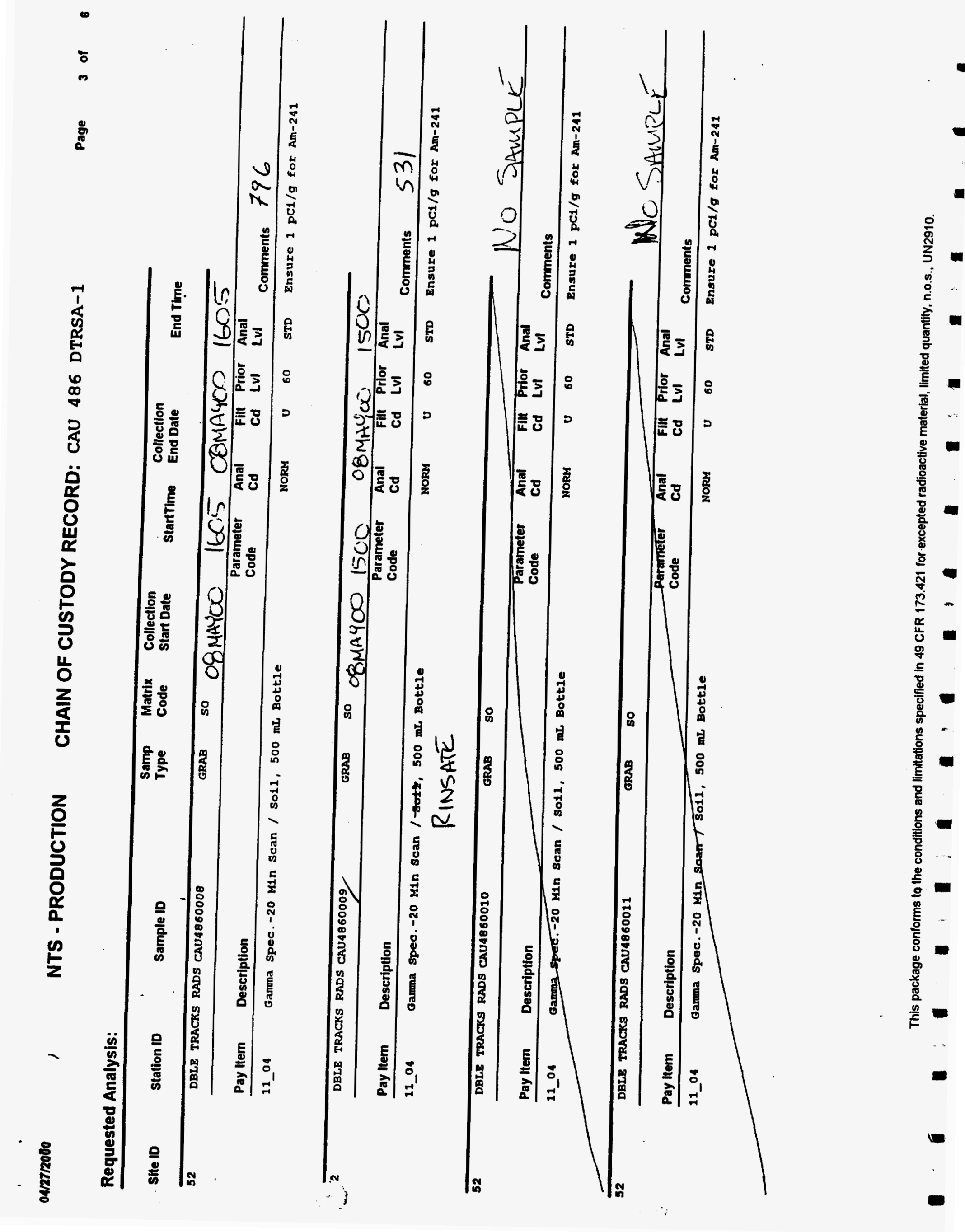




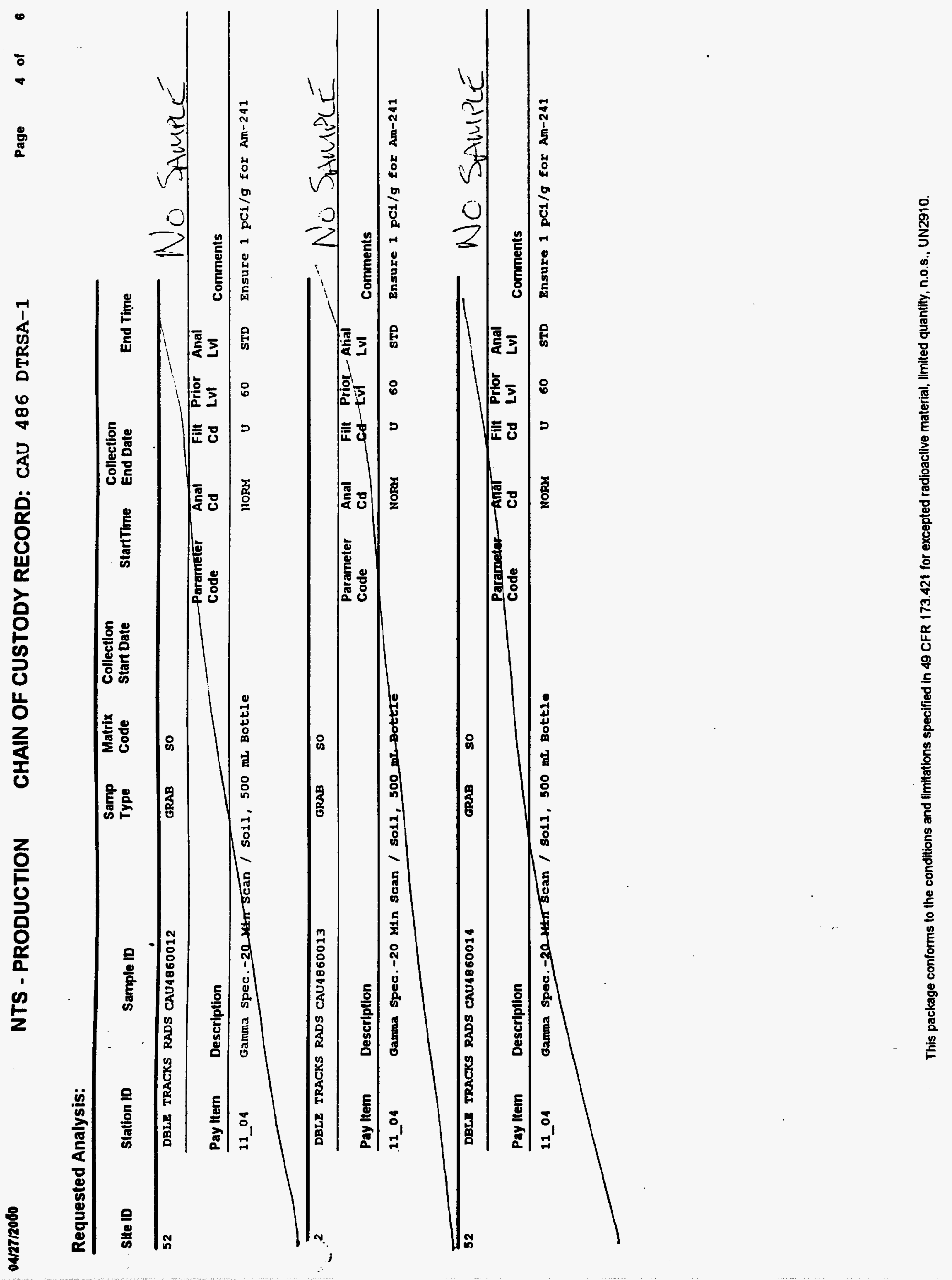



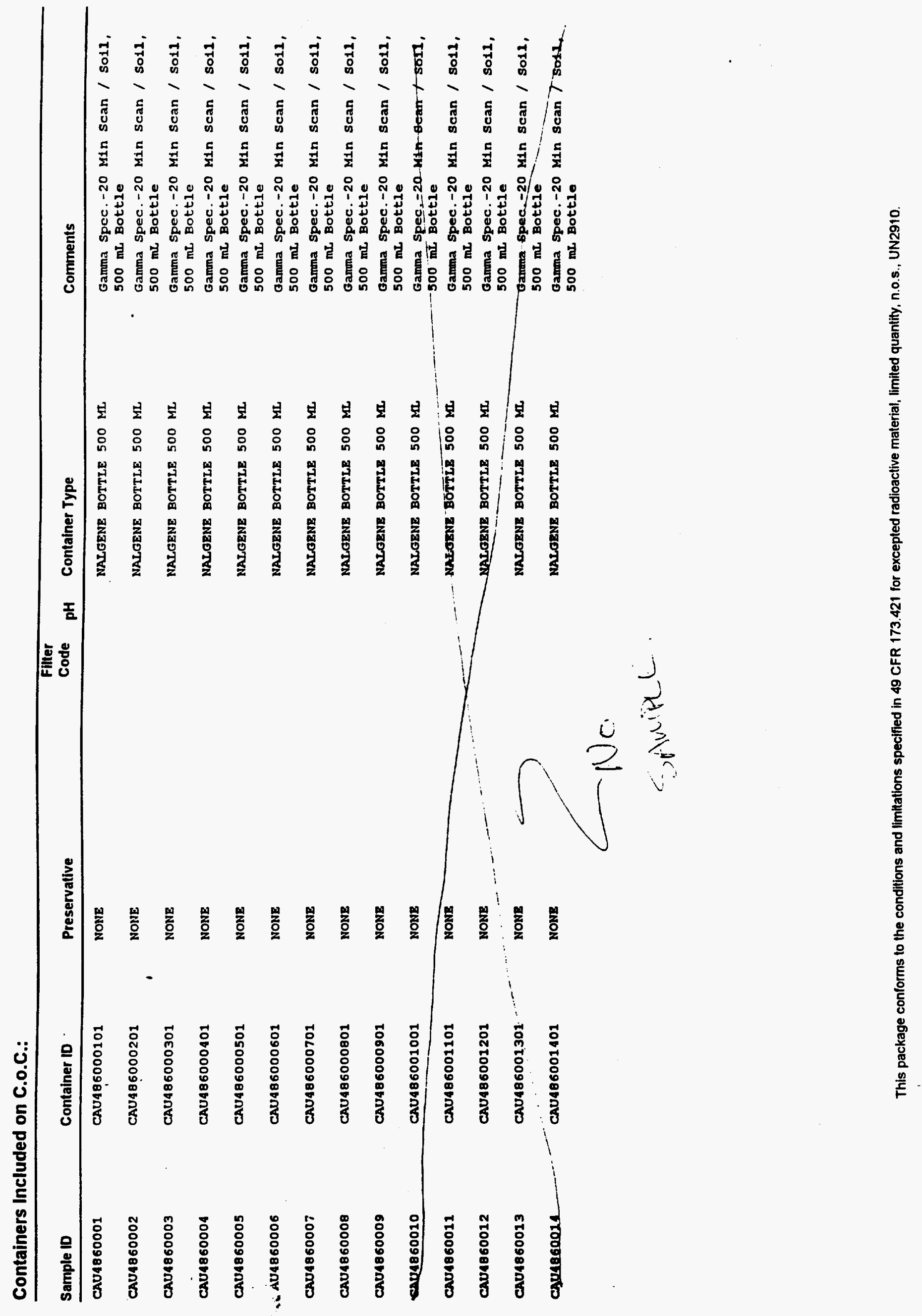


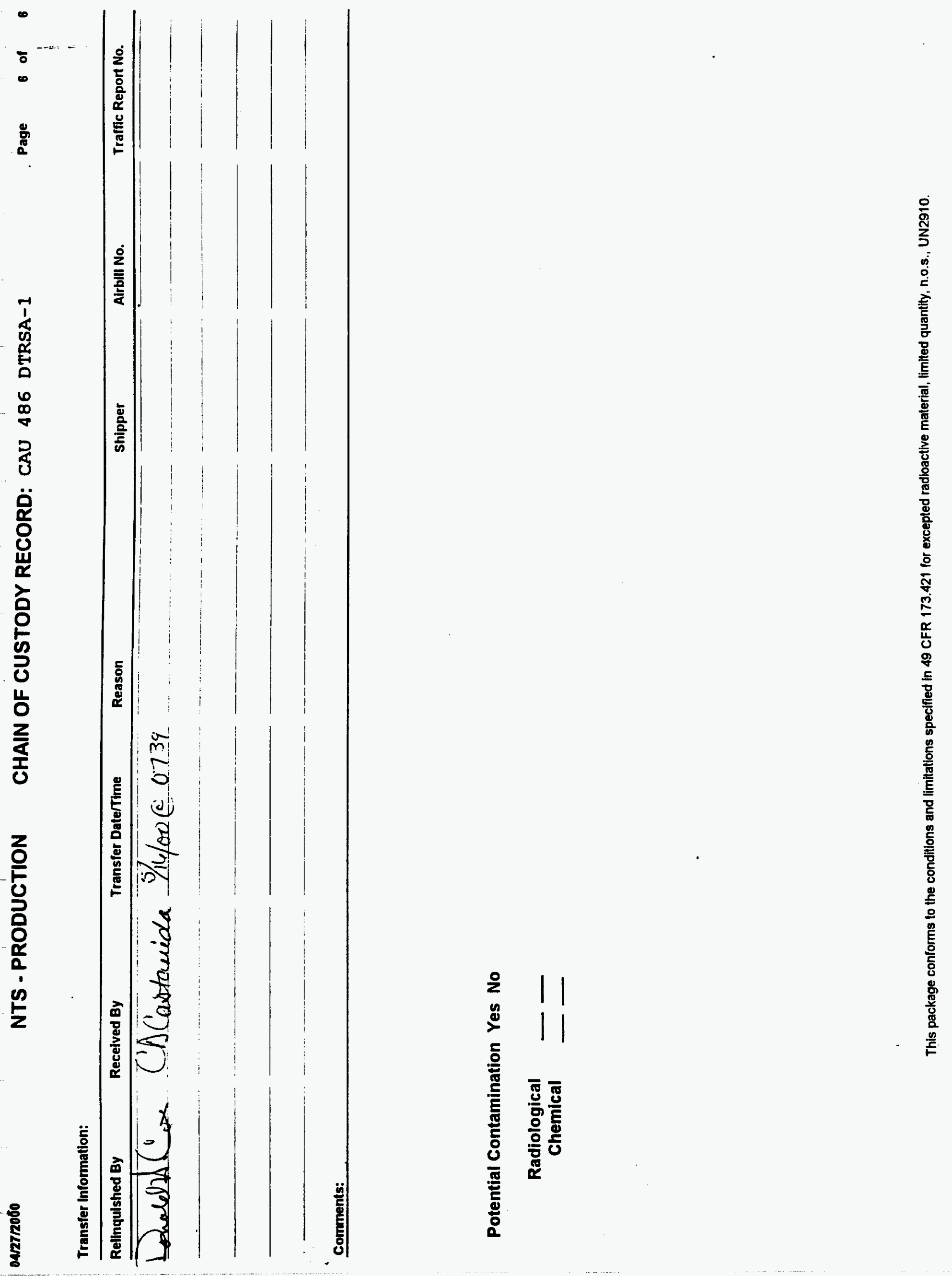





\section{Bechtel Nevada}

Interoffice Memorandum

To: W. F. Johnson

From: $\quad$ L. W. Hatcher Analytical Services Laboratory, 295-7109
Date: June 6, 2000

No.: 2154-LH-00-0448a

\section{Subject: DATA REPORT FOR SAMPLE DELIVERY GROUP (SDG) D321-AMENDED} Project No. 04001

Analytical Services Laboratory's (ASL) data results for the gamma spectroscopy analyses for the eight soil samples and one water sample submitted to the laboratory on May 16, 2000 are included with a copy of this memorandum to J. L. Smith. The service statement summarizing the costs and work performed by the Analytical Services Laboratory is also included.

A Bechtel Environmental Integrated Data Management System (BEIDMS) deliverable was also requested for this sample set. The BEIDMS electronic data file was loaded on June 1, 2000 in BEIDMS under document identifier "CAU 486 DTRSA-1".

Please direct any questions you may have to your Client Service Representative, Ted Redding, at 295-7220.

LWH:mer

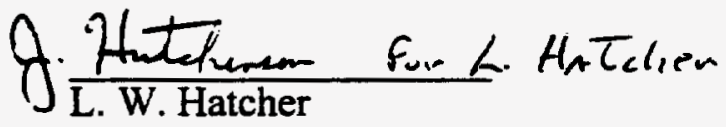

Subject Code: ENV3

cc: Correspondence Control, NLV008

S.M. Parsons-DePry (results enc.), NTS306

D. M. Van Etten NLV082

ASL D321, (results enc.) NTS273 




\section{APPENDIX D}

\section{FIELD NOTES}




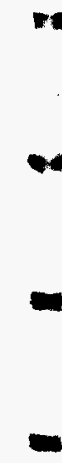

THIS PAGE INTENTIONALLY LEFT BLANK

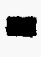




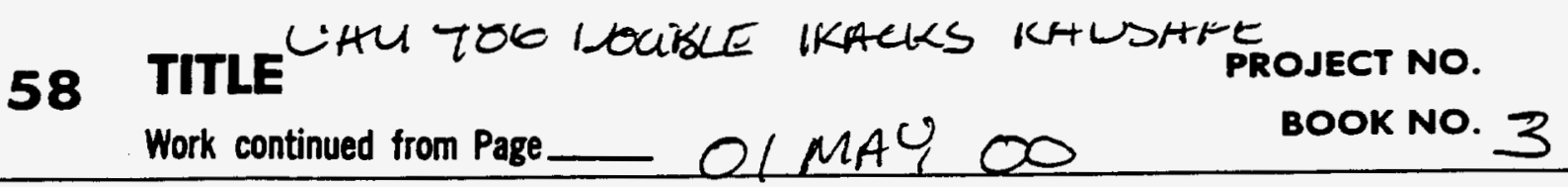

O830 Depart bor TFR

1100 - T TR, LUNCH

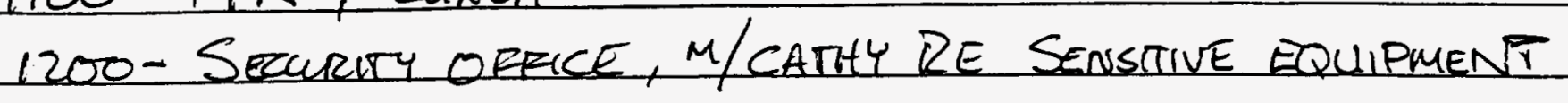
PASS

- over to radio shop m/ ken molke, pick up radios (3)

- back to main gate, pick up sat. telephone

- BACK to OFFice.

1330 - FO RM UP GROUP W/HEAVI EQUIPMENT, HEAD OUT TO DOUBLE TRACKS

- 1415 - ArRIVE DouBLE TRAEKS

1413-UNLOAD EQUIPMENT.

1500 - END ARRIVES, STAFF SGTWATTER - SR. AIRMAN RAIN. VERT RI TRAINING RECORDS. EON BEGINS SWEEP OF. RADSAFE AREA.

51525 -END REPORTS METALlIC SIGNATURE APPROX 30 METERS X 5 METERS CORRISFON DUG WITt CENTER OI BURIAL PT ("TOOBIG TO BE ABOMB") IS3O-EOD DEPARTS.

1600 - DePART DOURLE TRACKS.

1640- ARRIVE BN OFFICE.

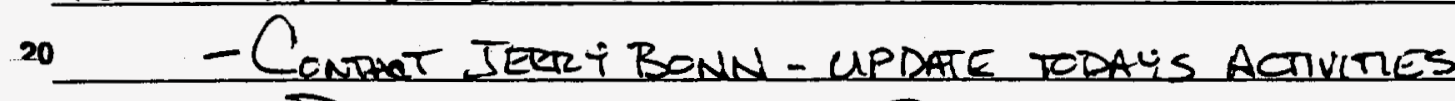

- Prep for tomorrow, Project documentation

1745 - SECURE OFELE.

25

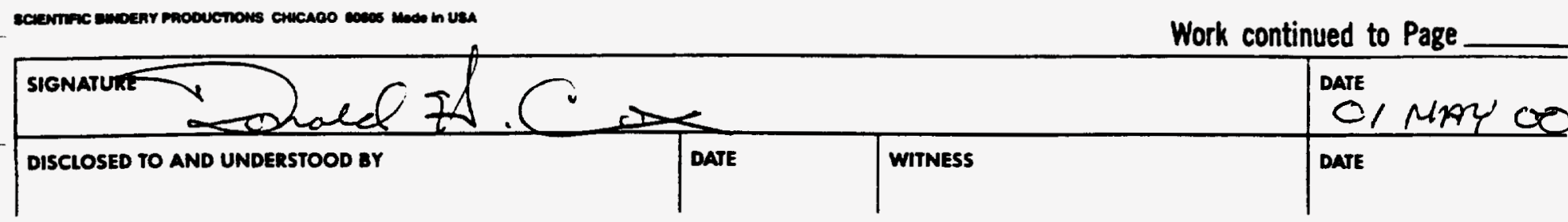


TITLE CAul 486, DeGreE Triers RadSAFE project no.

Work continued from Page - CZ MAY CC

BOOK NO. 3

GcC- FORM UP AT BN CERE, CLEAR, CALM, $\pm 60^{\circ} \mathrm{F}$

o700-Talleate safari briefing, Protect documentation.

- Review tophus activities, gas-up, prep for field, etc.

- Logistics; fuel for equipment, Portable toilets

5

- Water truck PTO, Problem - Construction to handLe

O800 - FORMED UIDMAIN GROUP, DEPART FOR DTRSA, ACCESS GATES

O900 - ARRIVE DIRSA, SET-UI, PREP EQUIPMENT, STE WALK.

- Discuss excavation to start, orient on original burial pis

- Paint surface, \& Pro's; START With \#t2, 61, GC of

10 Burial PIT LookING NORTH 4 WEST, 3 bORING, STAKES, ET,.

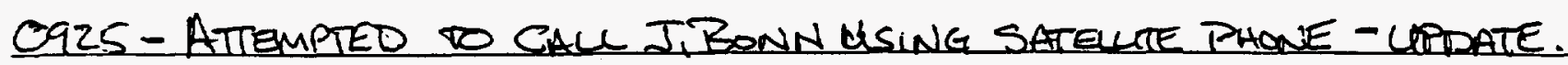

O940-REVIEN RIP, SIGNATURES, OPERATOR-I MM FISHER \& HP-CRAIG LYONS SITT-UP,

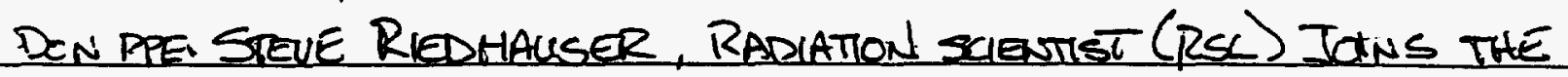
Entry TEAM.

151000 - ENTRY TEAM INTO EXCLUSION ZONE (EZ). BACKHOE INTO EZ

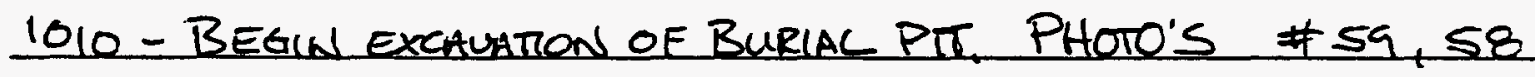

- using fiddler's in excavation, Photo \#57, Stock Pluming cleanFILL AT SOUTH END OF EXCAVATION.

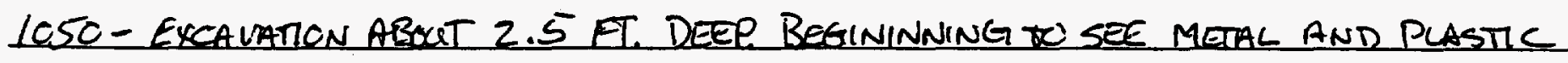
20 scraps, no elevated readings en aider yet. Continue to stockpile CLEAN FILL AT SOUTH END OF EXCAVATION.

HIC - AT APPREXMATELY 3.0 FT BES. WIRE, WOOD PLASTIC OBSERVED

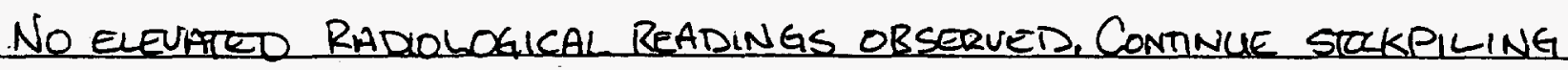
OF CLEAN FILL. PHOTO \#56 Looking SOUTH.

${ }^{25} \mathrm{izOO}-\mathrm{LaNCH}$

"Power bank"

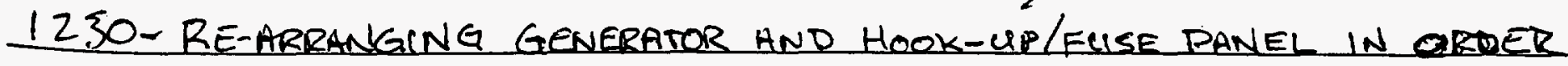
TO FACILITATE SPOTtING RAD COUNTING/LUNCH TRAILER.

- C. Lions, B. TenLPLETON AND I meEt to DISCLSS operation

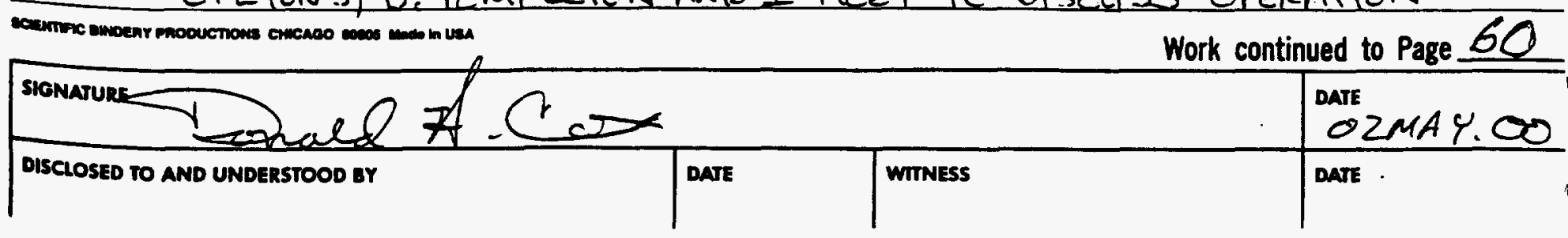


60 TITLE CAM 486 DoCILE TRACKS RADSAFE PROJECT No.

Work continued from Page 59 OZ MAY 00 BOOK NO. 3

MEETING TO DISCUSS OPERATIONS, CONTINUED - WE WILL CLEAN FLOR OF EXCAVATION, AND MOVE CLEAN-FILL TO NE CORNER OF OR EZ, AND THEN POTHOlE THE EXPECTED DEBRIS LOCATION. FISHER BACK INTO EZ 1300 - Begin cleaning excavation and moving cllan-Flll.

$51359-C$. LYONS AND D. COOPER SUTTUP ENTER EZ.

1412 -START FIRST POTHOLE

- STEVE RIEDHAULER DONS POE 4 ENTERS ER

1430 - Pothole EXcavate INTO DEBRIS AT MIDDLE BOREHOLE; "NB3" LOCATION. WE ARE OBUOUSLY IN THE DEBRIS-LAYER PORTION of the Burial PIT excavation. SeE photo \#55.

OVER ALL EXCAVATION IS APPROXIMATELY LOFT $X 50$ FT

"Floor of EXCAVATON IS APPROXIMATELY $20 \mathrm{FT} \times 30 \mathrm{FT} \times 3.5 \mathrm{FT}$ "Pothole" is APPROX matzo 6.5- 7 ET DEEP.

NOTE NOTE SlUG HT ELCUANON IN RAD (IZOODPM) IN ONE SMALL AREA OF DEBRIS, AND THAT IS FL L CONSIDERING THE AMOUNT OF TRASH EXPOSED.

1445 - Contacted I. BonN Re Confirmation of pLAN.

- Continue with work PLAN.

$150 C$ - MOVED THE FIRST B-25 INTO THE EZ, FILLED THE B-25 WITH SOIL AND DEBRIS.

1515 - InSTALLED THE HPGE DETECTOR OVER B-25, FIRT 1FT LIFTNO INDICATIONS CF ACTUTYY, FILLED B-25 WITH SECOND LIFT, " " " " " " " THIRD LIFT, " " " "ORANG OF THE THREE INSTRUMENTS

25 (ELECTRA, EIDER, OR HPGE)

1530 - ALL DEBRIS AT THIS POINT IS TRASH, NO HOTLINE MATERIAL

1545 - MOVED BACKHOE TO EAST SI OE OF TRENCH, EXTEND WIDTH OF

* High Purity Germanium

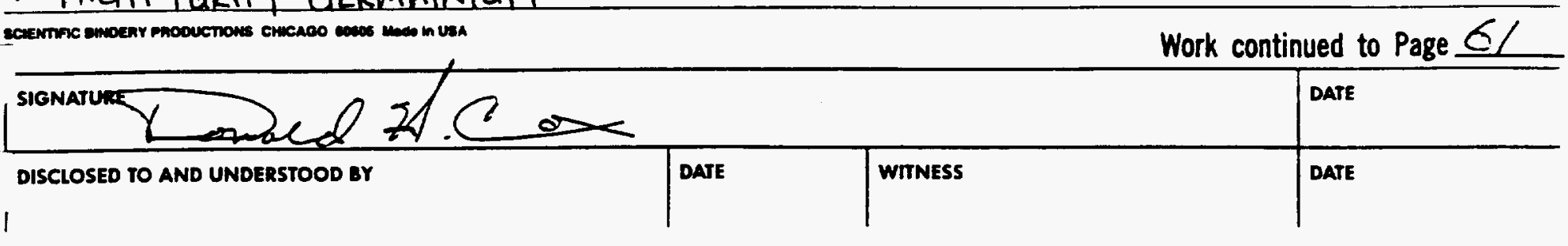


TITLECAU 486 DOUBLE TRACKS RADSAFE PROJECT NO.

Work continued from Page 60 OZ MAY. OO BOOK NO. 3

61

1545-CONTINUED, EXTENDING WIDTH OF TRENCH IN THE AREA OF

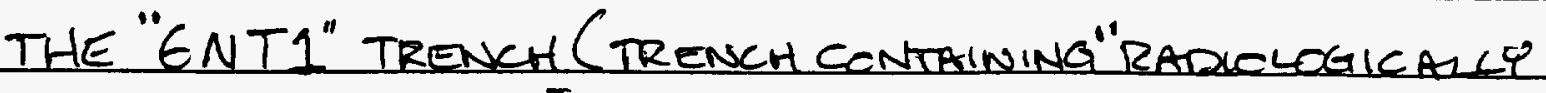
Contaminated DERRIS"). ONE BAg has BEEN Found WITH RADON ARCHED, BUT IT CONSISTS OF TRASH $(x)$

5 NOT HOTLINE WAST, AND THERE IS NO ELEVATED RADIO LOGICAL

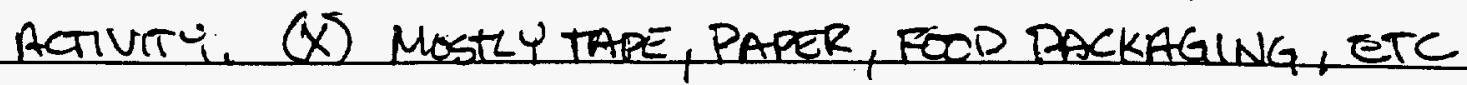

1600 - MOVED BACKHOE TO SOUTH SIDE OF EXCAVATION, EXTEND LENGTH v excavation OF TO SOUTH.

1615- MOVED BACKHHE TO NORTH SIDE OF EXCAUATION, 10

CUT EAST WEST TRENCH ACROSS NORTH END OFEXCAULATON.

1630 - Three baGS OF TRASH OBSERVED IN EXCAVATION.

- Secure excavation for the day.

- Frisk crew out of ez, breakdown location,

15 LOCK UP EQUIPMENT IN TRAILER,

1700 - SECURE SITE DEPART

1710- LEAUE RANGE IIN

$\$ 550$ 1750 -BAck to OFFiCe, Project Documentation,

20

25

* "Contaminated Material"

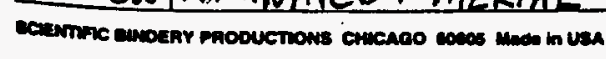

Work continued to Page

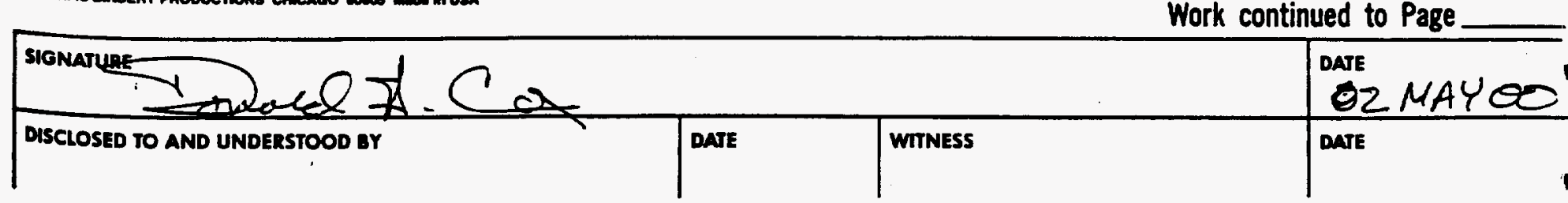


62 TITLE CAUl 486 DouBLE TRACKS RADSAFE

PROJECT NO.

MAYDHO

BOOK NO. 3

OG3C-PICKLIP LUNCHES, TO OFFICE, PROJECT DOCUMENTATION

OE 45- TELECON W/ Jib BONN - CRERATIONS.

o700-Tallgate safety Briefing, calls to news Range CoOrDinator, logistics

50730-FORM-UP GROUP \& HOAD FOR DOUBLE TRACKS.

ORO5-ON SITE, SET-UIP, CleAR, LT BREEZE, $\pm 70^{\circ} \mathrm{F}$

- Met w/ C. LYON's we decided $\pi$; 1 Set ONE B-25 CFFtr.

THE SIDE FOR ANYTHING OBVIOUS RAD MATERIAL OR INDICATING ELEVIATEO RAD. 2. IN CRIDERTC CONSERIE THE B-25'S WE WUL ATTEMPT TO SOMEWHAT SEPERATE THE MRTORITH OF THE CLEAN SOL FROM THE OTHER DERRIS.

OB45-TELECON FROM RANGE SCHEDULING RE SCHEDULE, TODAY THRU FRIDAY: TOMORROW WE WILL BE VISITED BY A HELICOPTER OPERATIc 100 FT AGR I TOLD RANGE SCHEDULING WE DID NOT HALE A PROBLEM WITH THIS OPERATED.

- STAGing B-255, PHotos \#S 51,52, \$53.

3930 -WATER TRLICK ON STE- WET DOWN EXCAVATON I IMMEDIATE SURROUNDING AREA.

O940-BEgIN LOADING B-255, SEGMENTING MATERIAL, PLASTK, WIRE, ETC., From Sold.

$D$ He

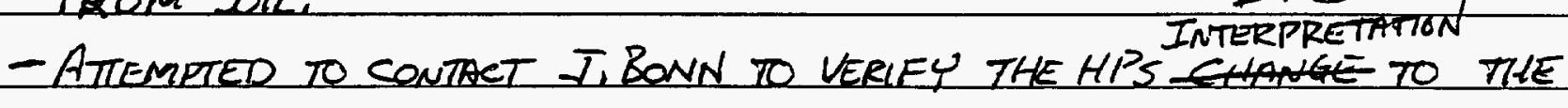
WORK INSTRUCTORS, - NEGATIVE CONTACT.

1030- CNE BAG SEPERATED TROM ALL THE OTHER DEBRIS THAT IS MARRKEDD WITH CONTAMINATION TAPE, HP MEASURES ACTW'TH AT 4000 COUNT/SEC.; 25 ON THE FILLER. 9 PHOTOS ( 41 - SCI) OF OPERATION AT THIS POINT, ExcavatiNG, DUST CONTROL, TRASH SEPARATICN, ETC.

1115 - FouND ONE BAG MEASURIN'S 500 COUNTS/ SEC, AND TWO BLACK TOTES MEASURING, EOO\$700 COUNIS/SEC RESPECTIVELY.

Work continued to Page 63
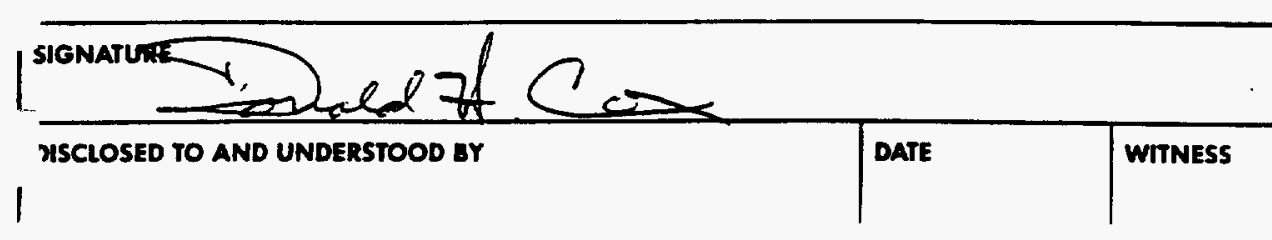
Dark 
TITLE CAUl 486 DOUBLE TRACKS RADSAFE PROJECT NO.

63

Work continued from Page 62 C3 MAY DUe

$1200-$ LuNCH

1230 - OPERATOR DENS PPI - FRISK OUT FORKLIFT IN PREPARATION FOR SITE support facility Re-arrangement, B. Templeton presently IN ROUE $T$ STE WITH EECTRLIAN.

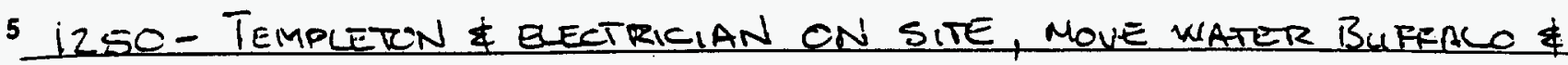
Generator, electrician Hooking up generator,

1315 - RESUME EXCAVATION/SEPERATION PROCESS

1335 -ONE BAG COllected WITH RADCON TAPE, CONTAINING MUSTLY TAPENo elevated activity apPARENT,

101340 - ONE LARGE bag of ANTI-CS ANO totes COLLECTED and AdDED to "RAD CONTAMINATED" B-25, No elevated ACTIVITY ADPARENT.

1350 - TWO B-2.55 ARE NOW FULL, CHANGE -OUT WITH EMPTY BOWS,

- THE TWO FULC B-25S moved to THE HPGE testing area,

1414 - RESUME EXCIUVATION/SEPQRRTION PRECESS.

$1515 C C-A / A$, PHOTOS\# $38,39,40,41$.

IS /5 - CNE BAG OF GLOVES RECOVERED, NCELUATED ACTUVITI APPARENT.

- ONE BAG of LATEX Gloves Recovered, 370 Counts/ sec Indicated.

1615 - ContinuE excavation/seperation process.

- Clean-upexcavation, secure location.

$20163 E-$ DEPART RANGE $71 N$.

1700-Arrive Bnoface, area 3.

- Project Documentation.

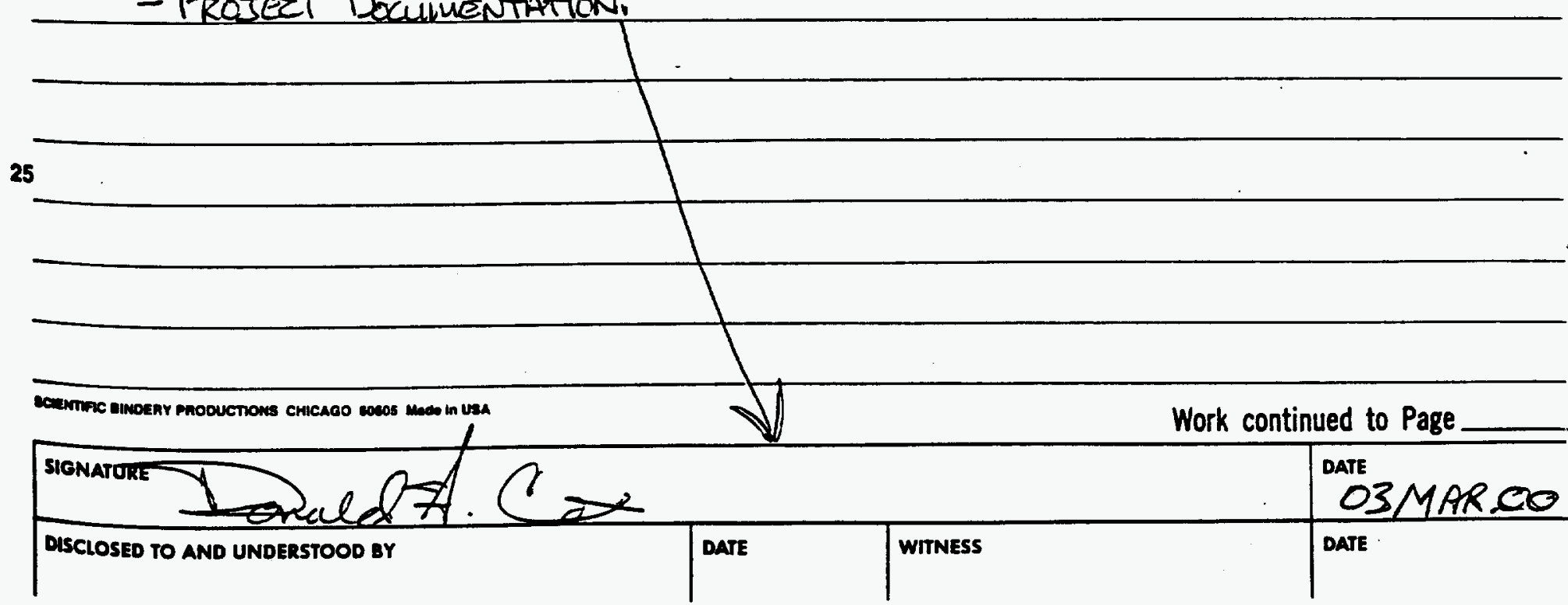


64 Title $Q A 4486$ Double Tracks Radsafe project no.

Work continued from Page $=04$ MAY. 00 BOOK NO. 3

0630 - Pick vip Lunches, to OFFice, Protect Documentation.

otoo-Tatlgate Safety Meting. Form-UP Group -

O730-DEAART FOR DOUBLE TRACKS.

O810 -ARRIVE DOUBLE TRACK STE, PREP TO BEGIN WORK.

5C830-Begw Exeavation/Septration Procedure

O850-One Bag (Plastid) containing RAD TAPE, 700 counts/ SEc

o9os-Continue excavation / serration Procedure, PHoto's \#35,36,37,38...

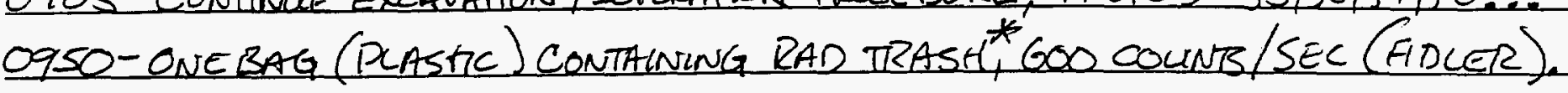
* Rubber, pope, woOd, etc.

10

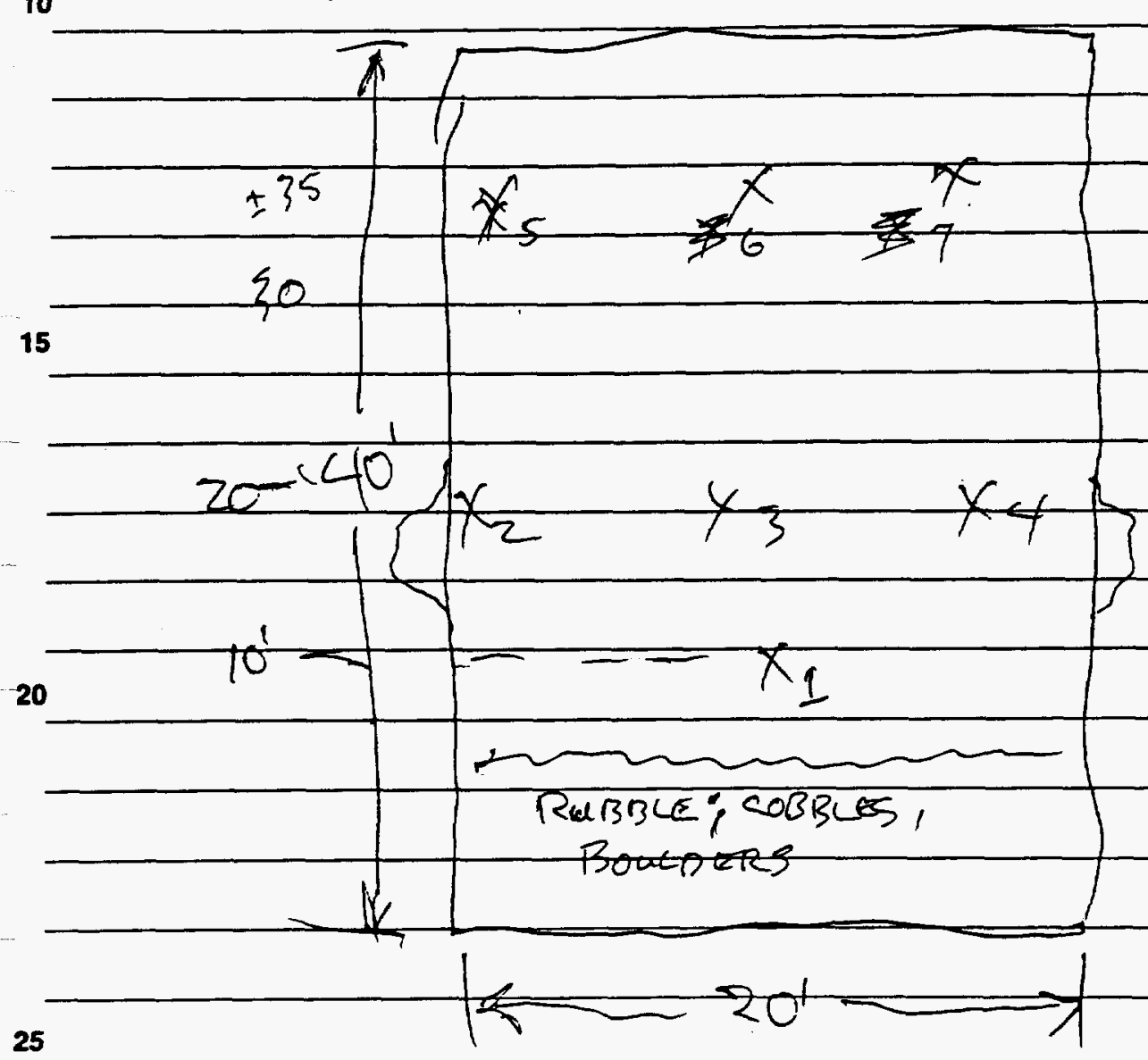

SKETCH 1

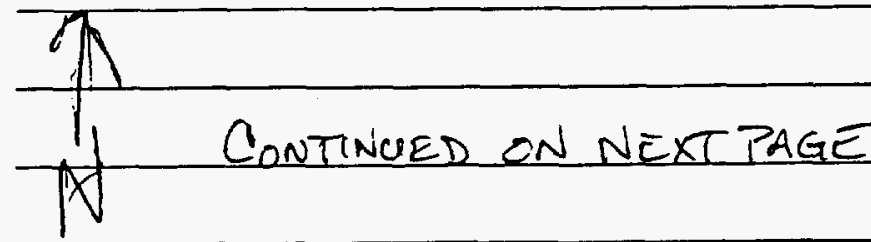

\begin{tabular}{|l|l|l|l|l|}
\hline SIGNATUKE Work continued to Page 65 \\
\hline DISCLOSED TO AND UNDERSTOOD BY
\end{tabular}


TITLECAU 486 DOUBLETRACKS RADSAFE PROJECT NO.
Work continued from Page GE OU MAY $O$

65

1000 -DON PPI, ENTER EZ, LAYOUT VERIFICATiON SAMPLES, MEASURE excavation, SEe sketcH on page gt

- Clean up floor of excavation

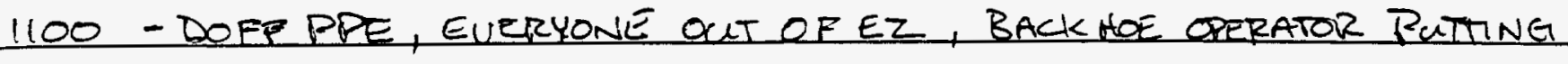
BERM AROUND EXchuation EOR THE wEEKEND.

$1200-1$ un ch/ SECure Location

- Head back to BN -Office.

$1330-B N$ OFFICE

- Project documentation, telecoms, logistics.

101430 - Secure and depart location.

15

20

25

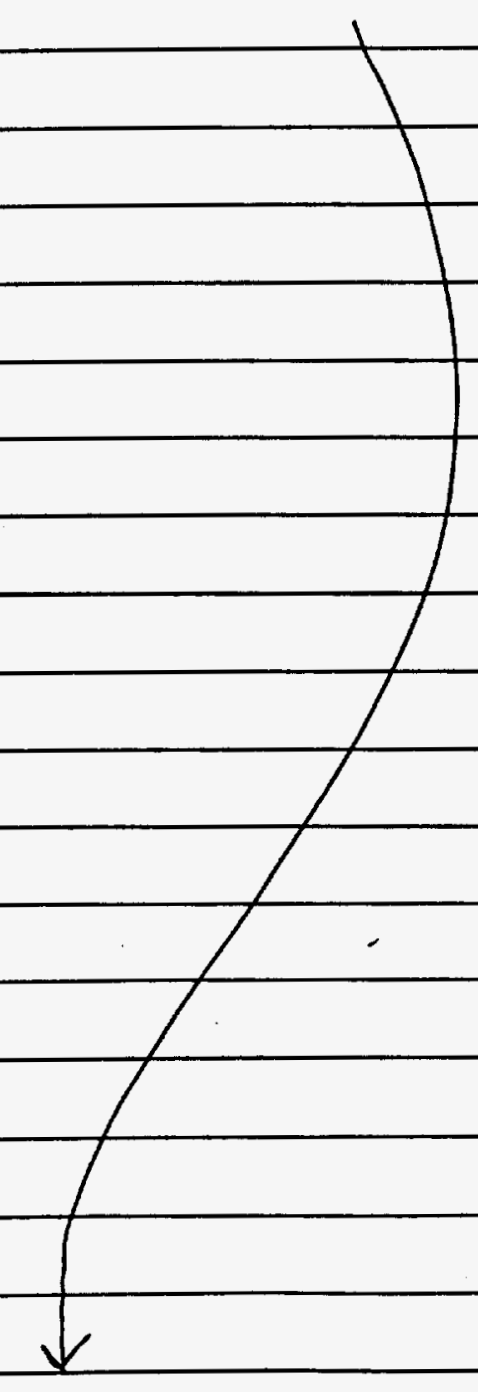

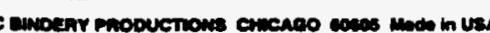

Work continued to Page

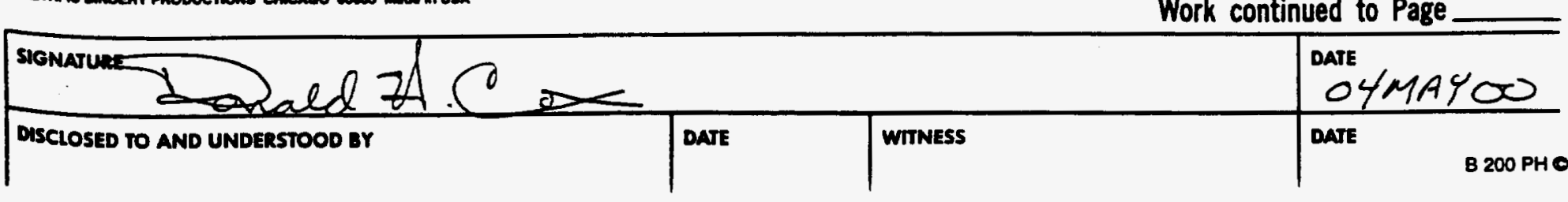


66 TITLE CAM 486 DUULLETRACKS RADSAFE PROJECT NO.

Work continued from Page — OBMAY OO

BOOK NO. 3

0830-ARRUV TR, Pick UP CAMERA PERMT, STOPPED BS the BASE

Commanders office, tHE Col. WAS wOT IN.

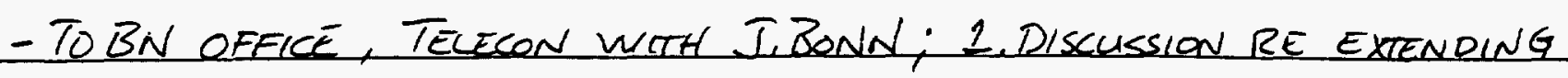

THE EXCAvation, 2. Logistics.

5

- Various telecons; BlackTack-RANGe ScheDULing, LASERs working IN RANGE 71 SOUTH, THIS AFTERNOON.

DUe

1050 - TAIlGATE SAEETU MEETING, REVIEW TODAYS TASKS, HABERMAS E-MAIL.

1130 - LUNCH

$1230-$ FORM-UP \& HEAD FOR DOUBLE TRACKS.

10 - WAITNG on Gate Access (CACTUS SPRING GATE) (AFTER BEING CHIDED LAST WEEK BU CACTUS, FOR NOT GIVING THINK ENOUGH TIME, I CALLED TODAY FROM THE BN OFFER PARKING LOT, AND WE STR HAD TO WRIT FOR THEM AT THE GATE)

1330-ARRIVE at DOURLE TRACKS STE, PREP, DON PPE, CHECK E EXUPMENT 15

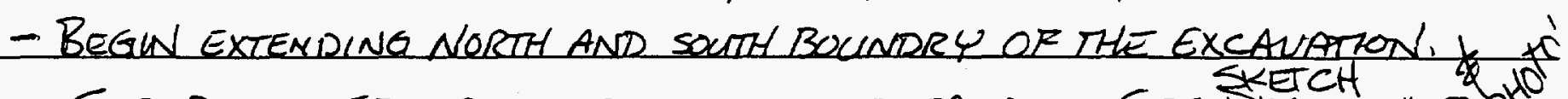
1530 - SuITUP, into EZ, Excavate AND collect SAMPLES. SeE KETCH 1535 - SAMPLE \#1, 1540-SAMPCE HZ, 1545-SAMPLE \#3, 1550- SAMPLE \#4

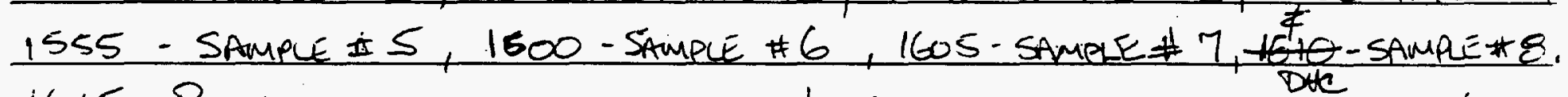
1615 - PUSH IN CORNERS OF EXCAVATION, ALL SAMPLES CHECKED LEITH 20 MPG DETCTOR AND THEN SW IED BYRCTS,

1650 - EvERYONE OUTOFEZ, SECURE AND DEPART LOCATOR,

1720 - BACK to BN Opelce. Proper Documentation.

25

SCENTHAC anNeEAY PAOOUCTONS CHICACO 20506 mede in UsA

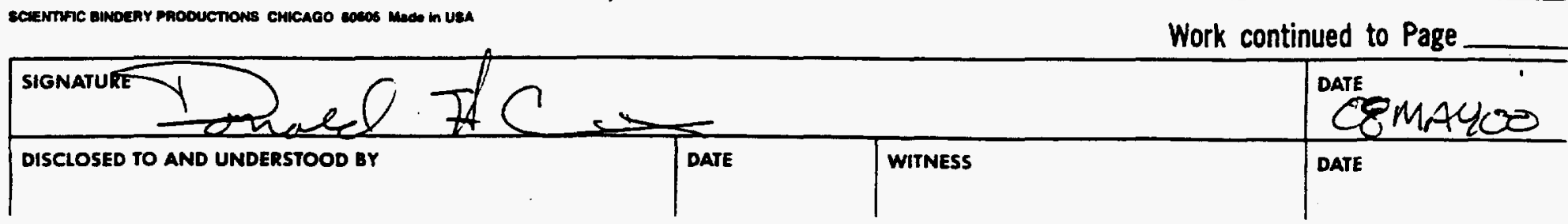


TITLE CAU 486 DourLLE TEACKS RADSA FE Work continued from Page 66

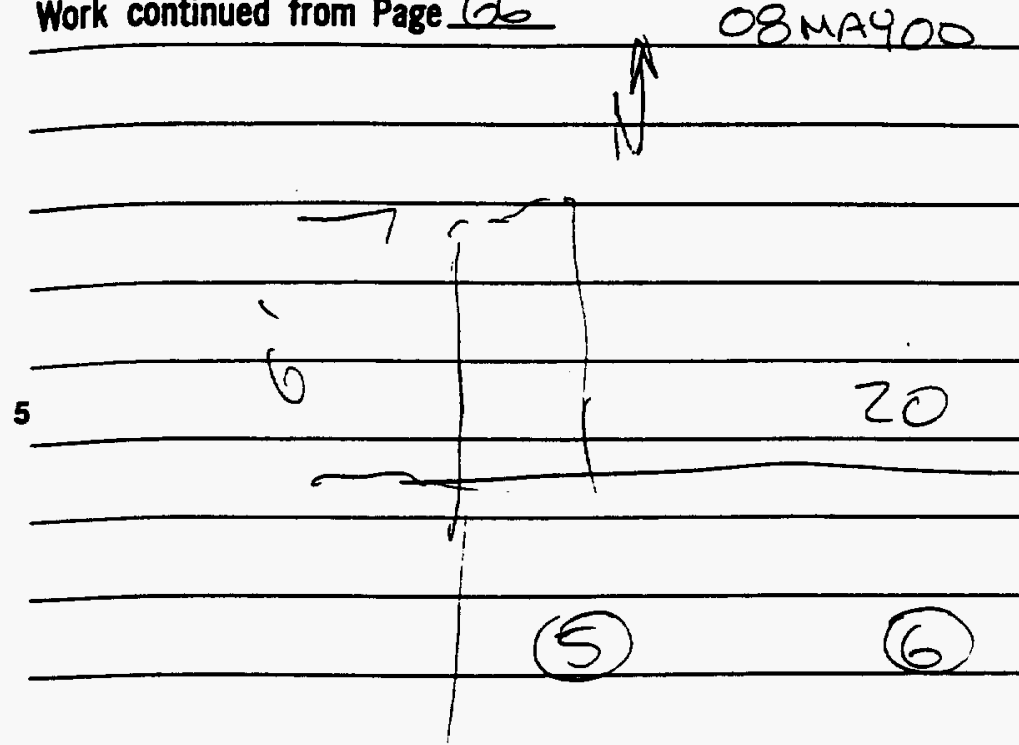

10

25

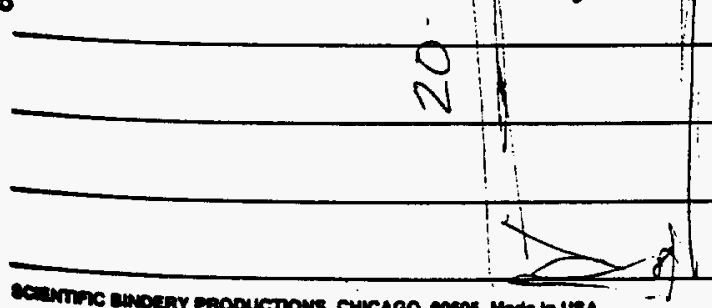

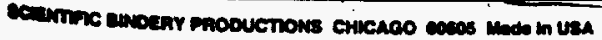

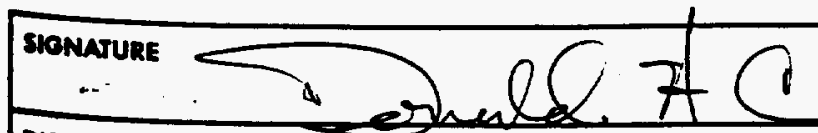

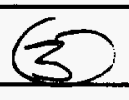

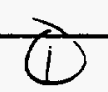

PROJECT NO.

BOOK NO. 3 
68 TITLE CAU 486, DoutsLE TRACKS RADSAFE PROJECT NO.

Work continued from Page__ OA MAY OO

BOOK NO. Z

0630-ForiM. UP AT BN OFFICE. Project DOCUMENTATION.

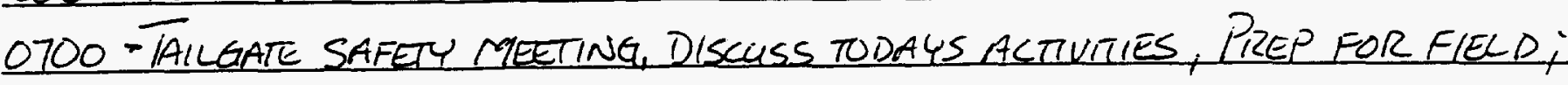

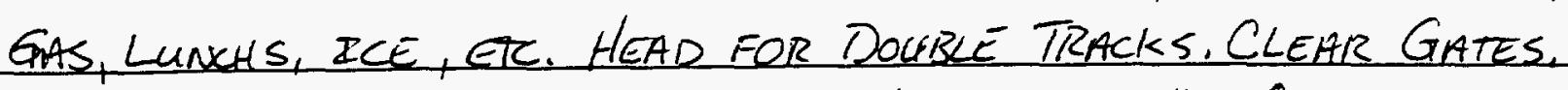

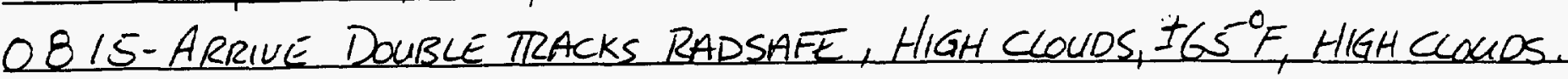

5 - OPERATOR DONS PE, HEADS INTO EZ, SERVICE EQUIPMENT

0830- Laborer dons Poe, into ez to assist operator, seperrate plastic from spoils PILE. Operator begins (CONTINUES) Back FiLLING EXCAVATION,

O910- RCT (R.D.) DONS PPE, ACcess "DECONTAMINATION FACIUTY" FOR 10 "STOMP AND Tromp" "N ORDER TO DEPOST AREA.

1000 -ESCORTED RSI OFF RANGE TIN.

1020 -BLEW FRONT LEET TIRE ON BACKHLE, CONTACTED B. TEMALETON AT THE OFFICE RE REPAIR,

1130 - FuEL TRUCK AND ELECTRLAN ON SIIE. DISSONNEAT TRAILER.

${ }^{15} 1145$ - OPERATOR COMES OUT OF EZ FOR LUNCH

1240-OPERATOR \& LABORER INTO EZ, USE BACKHOE BUCKET DO TRANSFER SOIL FRom stock PILe to excavation. Furl Truck terotrican depart (escorted)

- Back hoe Tire Logistics,

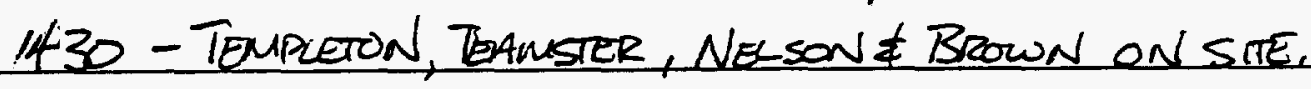

20

- hook-up water buffalo. Various tire calls

1455 - Templeto, TEAmsters, NeLSON \& Brown Depart

- Begin WeIGHing B-25s.

1530 - BEGIN FILLING BODS

1600 - B-Z5S FILL E WIT SOIL, MOVE TO CONTAMINATED STORAGE AREA FOR

25 SWIPES

- MOVE BACK HOE TO FENCE, SWIPE TIRE FOR REPAIR

1630 -SECURE AND DEPART LOCATION

* BOT, SCOOT, AND BOOGIE, BY BROOKS $\neq$ DUNN"(R.D,)

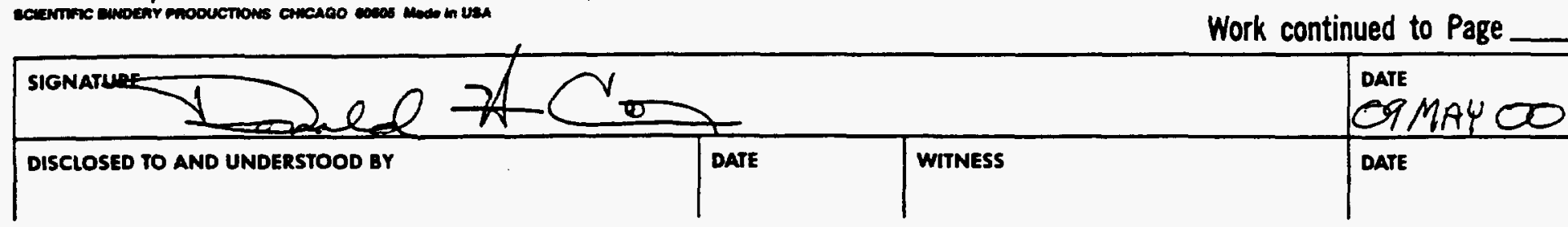


TITLE CAU 486, DOUBLE TRACKS RADSAFE

PROJECT NO.

Work continued from Page__ $10 \mathrm{MAY}$ -

BOOK NO. 3

69

OG30 -MET DAD TIRE AT MAIN GATE.

0700 - Back to BN OfFice, Logistics, TaIlgate safety Meeting.

0800 - Arrive Double racks site, CleAr, $\pm 60^{\circ} \mathrm{F}$, BreEzY.

- Prep to weigh b-25s. Tire repair complete *

52820 - BeGIN WeIGHING, B-25S.

- Setup to enter ex to Backfill excavation l.

o840 - Operator into ez to BAckfill excavation. PHOTOS

- Waste ops closing, securing B-25s for transportation.

- Laborers removing fence from old decontamination facility.

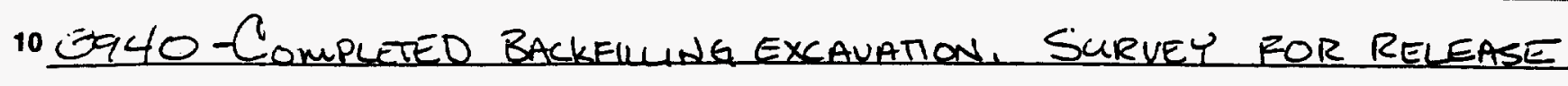

1010 - Water Down Excavation.

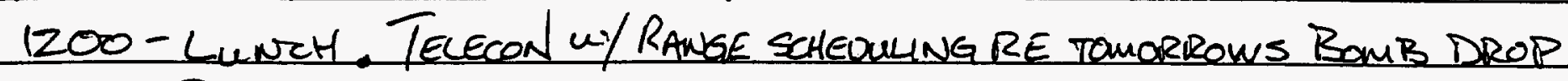

1230-PREP ROR LOADING B-25S. MiND INCREASING-BLOWING DUST

1300 -Trucks arrive, LOAD B-25s.

1514 - LOAD GENERATOR

1435 - FORK LFT AND TRUCK (TRATOR war l FLATIBED) TO LOAOWG RAMP ABOUT $4 \mathrm{MI}$ EAST OE DOUBLE TRACKS STE F

1500 - LOAD UP RCT/SUPPLY/TESTING EQUIPMENT TRAILER

1600- I DEPARTED STE ESCORINNG 3 TRUCKS AND 4 OTHER WORK VEHKLES

20 LEAVING BLLL AND OPERATOR + A LABORER \& HEATH FSAFETQ to PulL POSTS VISBILTT DOWN TO ABOUT $2 \mathrm{mi}$, $40-50 \mathrm{MPH}$ WINDS. $1630-B N$ OFFICE

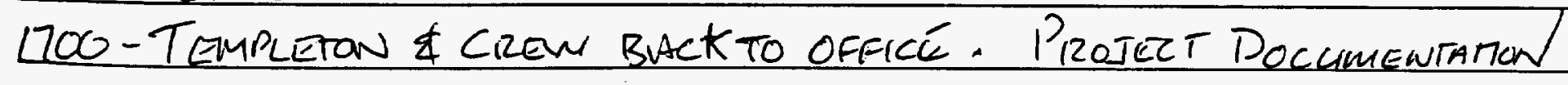

1745- Secure \& DERART OFF KE

25

* We where directed to use oed tire from Toto pah by suezanne, area 6 equip.

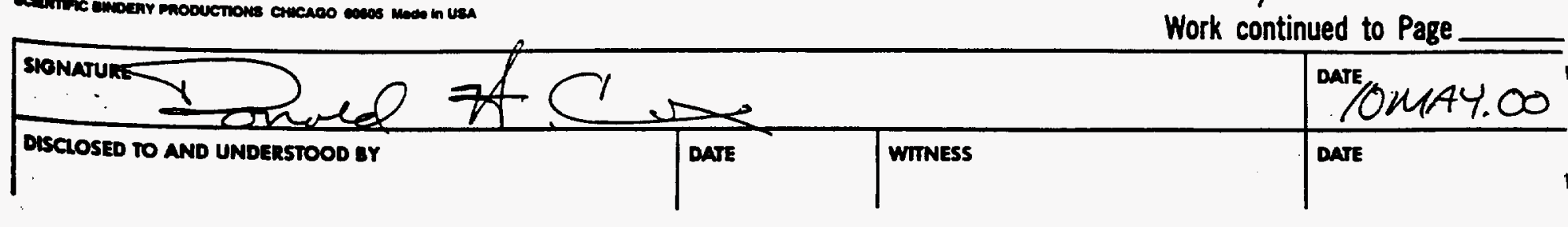





\section{APPENDIX E}

\section{COMMENT RESPONSE DOCUMENTATION}




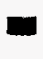




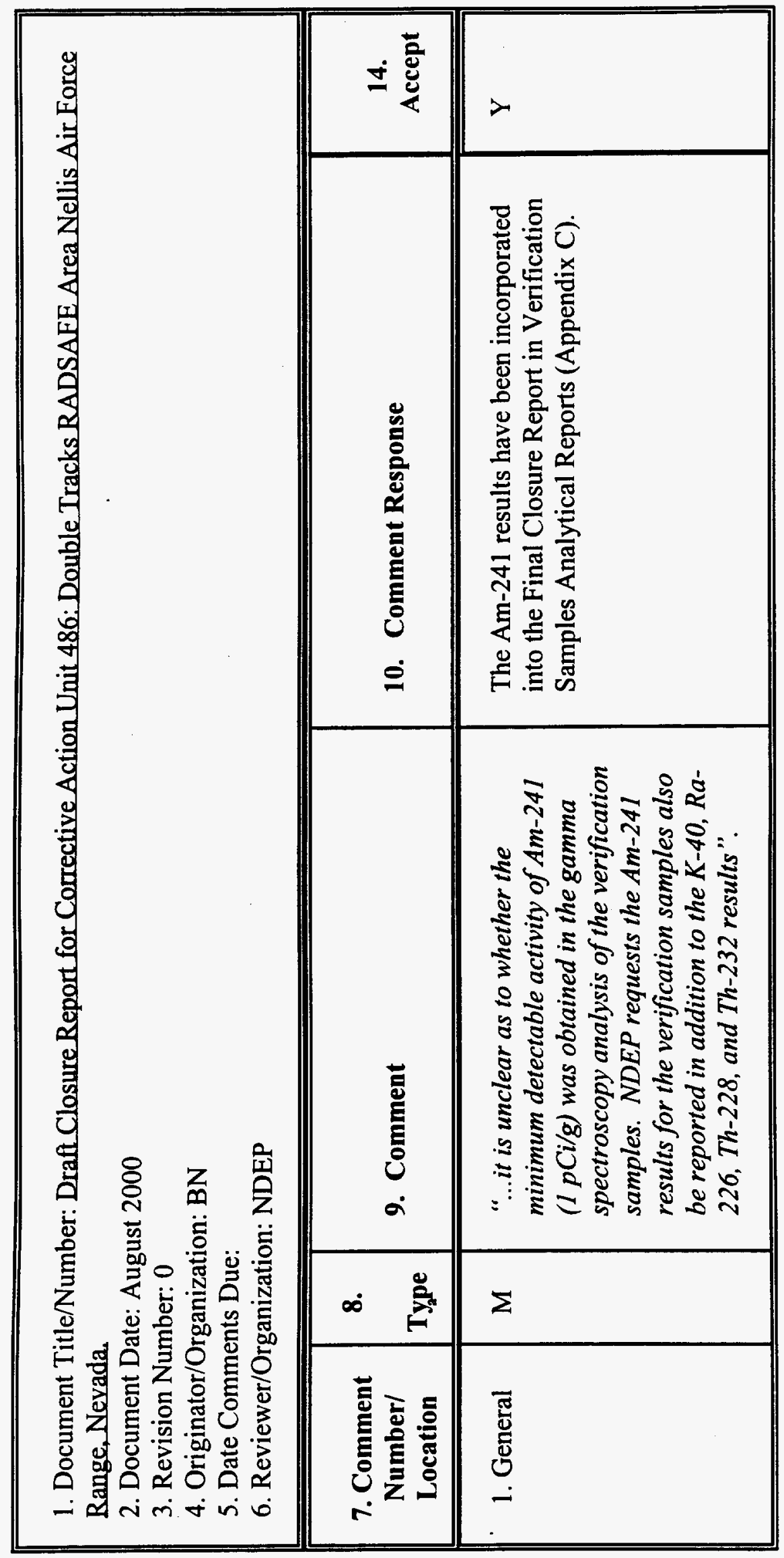

5
$\frac{5}{0}$
0
0
0

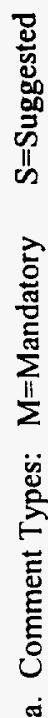




\section{DISTRIBUTION LIST}




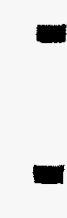




\section{DISTRIBUTION LIST}

*Provide copy of initial distribution or Revision 0; remainder of list gets Revision 0 if approved without changes. The entire list receives Revision 1, if issued.

\section{Nevada Division of Environmental Protection}

Paul Liebendorfer

2 (Controlled)*

Bureau of Federal Facilities

Division of Environmental Protection

333 W. Nye Lane, Room 13B

Carson City, NV 89706-0866

Mike McKinnon

1 (Controlled)*

Bureau of Federal Facilities

Division of Environmental Protection

Sawyer State Building

555 E. Washington, Suite 4300

Las Vegas, NV 89101-1049

\section{U.S. Department of Energy}

Janet Appenzeller-Wing

1 (Uncontrolled)*

Environmental Restoration Division

U.S. Department of Energy, Nevada Operations Office

P.O. Box $98518 \mathrm{M} / \mathrm{S} 505$

Las Vegas, NV 89193-8518

Kevin Cabble

1 (Uncontrolled)*

Environmental Restoration Division

U.S. Department of Energy, Nevada Operations Office

P.O. Box $98518 \mathrm{M} / \mathrm{S} 505$

Las Vegas, NV 89193-8518

Sabrina Lawrence

1 (Controlled)*

Environmental Restoration Division

U.S. Department of Energy, Nevada Operations Office

P.O. Box $98518 \mathrm{M} / \mathrm{S} 505$

Las Vegas, NV 89193-8518

U.S. Department of Energy

Office of Scientific and Technical Information

1 (Uncontrolled,

P.O. Box 62

Oak Ridge, TN 37831-0062 


\section{DISTRIBUTION LIST (continued)}

\section{U.S. Department of Energy (continued)}

U.S Department of Energy

1 (Uncontrolled)

Nevada Operations Office

Technical Information Resource Center

P.O. Box 98518, M/S 505

Las Vegas, NV 89193-8518

Manager, Southern Nevada

1 (Controlled) \&

FFACO Public Reading Room

1 (Uncontrolled)

P.O. Box 98521, M/S NLV040

Las Vegas, NV 89193-8521

Manager, Northern Nevada

1 (Uncontrolled)

FFACO Public Reading Room

Nevada State Library and Archives Federal Publications

100 North Stewart Street

Carson City, NV 89701-4284

\section{U.S. Air Force}

U.S. Air Force DOE Liaison Office

1 (Uncontrolled)*

DOE/Nevada Operations Office

P.O. Box 98518, M/S 505

Las Vegas, NV 89193-8518

Eloisa V. Hopper

3 (Controlled)* $^{*}$

U.S. Air Force

$99 \mathrm{ABW} / \mathrm{EM}$

4349 Duffer Dr., Suite 1601

Nellis AFB, NV 89191-7007

Major Roger Schofield

1 (Controlled)*

U.S. Air Force

AWFC RMO/RML

3770 Duffer Dr.

Nellis AFB, NV 89191-7001 


\section{DISTRIBUTION LIST (continued)}

\section{Sandia National Laboratory}

Vern Gabbard

1 (Controlled)

Sandia National Laboratory

Tonopah Test Range

Tonopah, NV.

\section{Bechtel Nevada}

Correspondance Control

1 (Uncontrolled)*

Bechtel Nevada

P.O. Box $98521 \mathrm{M} / \mathrm{S}$ NLV008

Las Vegas, NV 89193-8521

Environmental Management Library

1 (Uncontrolled)*

Bechtel Nevada

P.O. Box98521, M/S NLV080

Las Vegas, NV 89193-8521

Ann Heidema

1 (Uncontrolled)*

Bechtel Nevada

P.O. Box 98521, M/S NLV 022

Las Vegas, NV 89193-8521

Wayne Johnson

1 (Uncontrolled)*

Bechtel Nevada

P.O. Box 98521, M/S NTS306

Las Vegas, NV 89193-8521

Steve Nacht

1 (Uncontrolled)*

Bechtel Nevada

P.O. Box 98521, M/S NTS306

Las Vegas, NV 89193-8521

Dennis Gustafson

1 (Uncontrolled)*

Bechtel Nevada

P.O. Box 98521, M/S NTS306

Las Vegas, NV 89193-8521 
Date: January 3, 2001

\section{DISTRIBUTION LIST (continued)}

\section{Bechtel Nevada (continued)}

Don Cox

1 (Uncontrolled)*

Bechtel Nevada

P.O. Box 98521, M/S NTS306

Las Vegas, NV 89193-8521

Jan Cowley

1 (Uncontrolled)*

Bechtel Nevada

P.O. Box $98521 \mathrm{M} / \mathrm{S}$ NTS 110

Las Vegas, NV 89193-8521

\section{IT Corporation}

Lowell Wille

1 (Uncontrolled)*

IT Corporation

P.O. Box 93838, M/S NLV439

Las Vegas, NV 89193-3838

Dustin Wilson

1 (Uncontrolled)*

IT Corporation

P.O. Box 93838, M/S NLV439

Las Vegas, NV 89193-3838

IT FFACO Support Office

1 (Controlled)

IT Corporation

P.O. Box 93838, M/S NLV439

Las Vegas, NV 89193-3838 